\title{
YUCCA MOUNTAIN SITE CHARACTERIZATION PROJECT \\ EAST-WEST DRIFT SYSTEM SAFETY ANALYSIS \\ REVISION 02
}

June 8, 1999

Document Identifier: BAB000000-01717-0200-00004 Rev 02

Prepared for:

U. S. Department of Energy

Yucca Mountain Site Characterization Project Office

P.O. Box 98608

Las Vegas, Nevada 89193-8608

Prepared by:

TRW Environmental Safety Systems, Inc.

1180 Town Center Drive

Las Vegas, Nevada 89134 
DI: BAB000000-01717-0200-00004 Rev 02

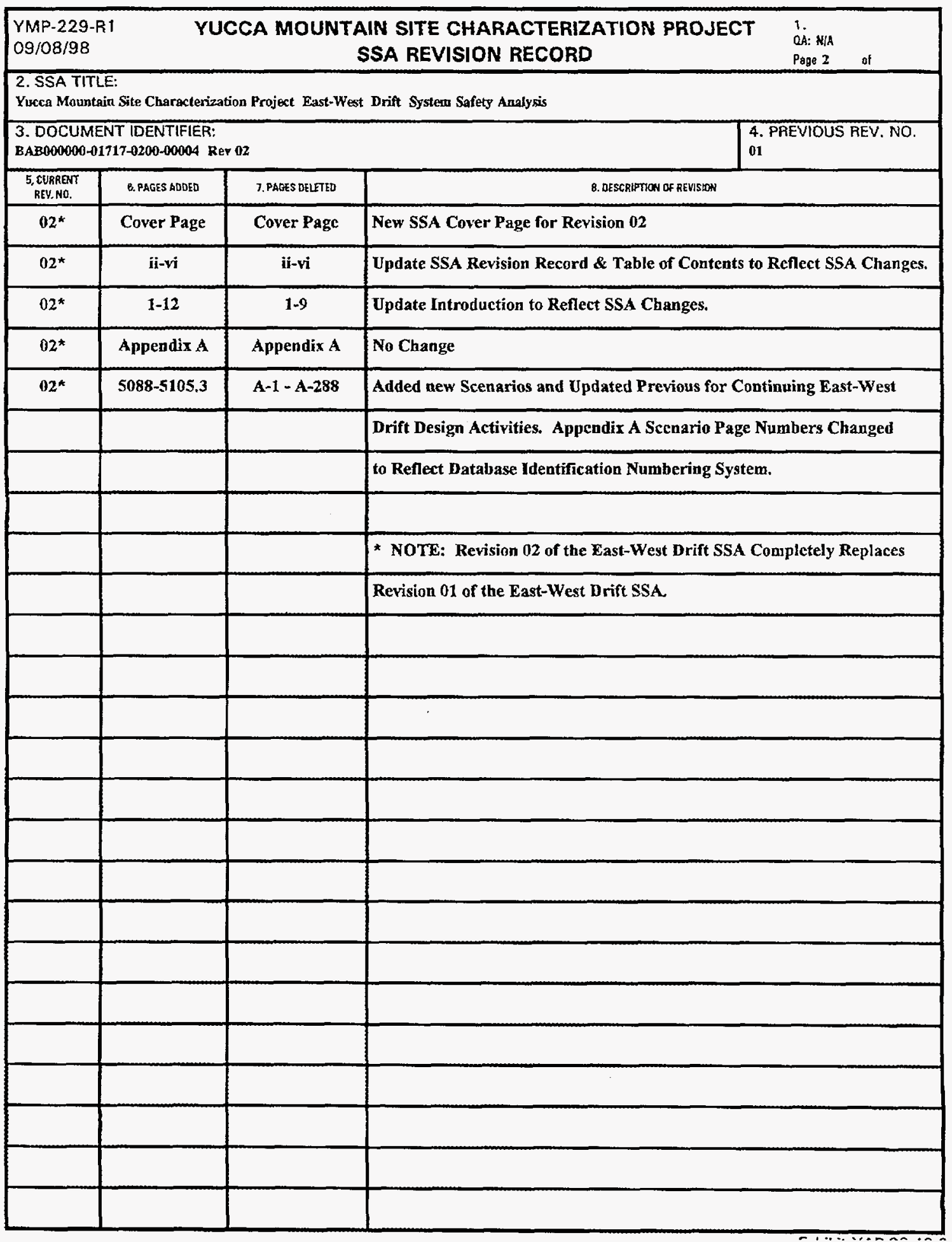




\section{DI: BAB000000-01717-0200-00004 Rev 02}

\section{TABLE OF CONTENTS}

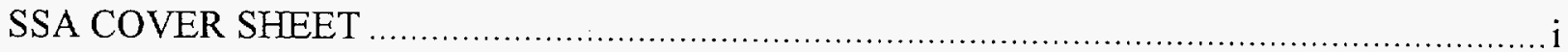

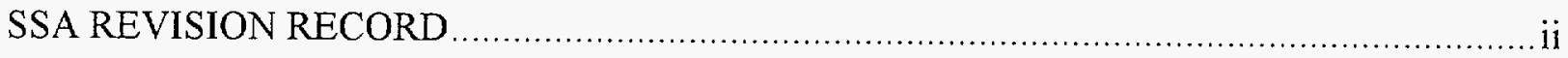

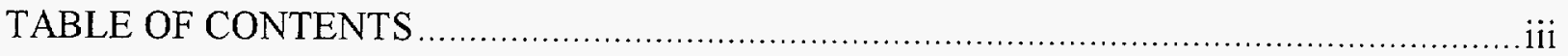

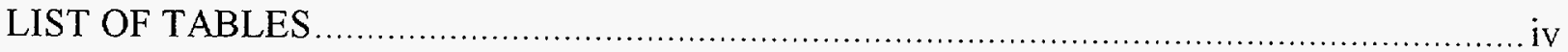

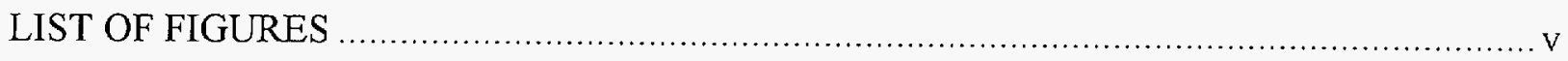

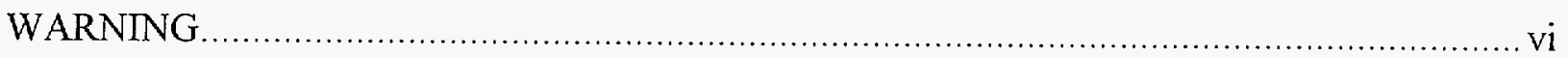

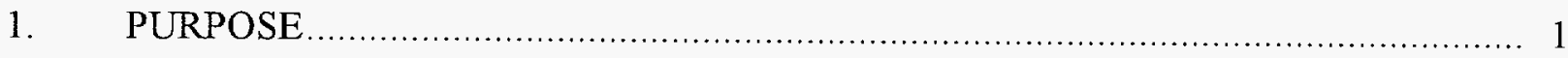

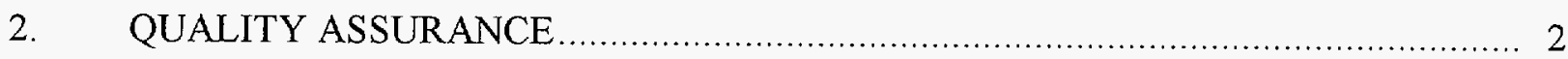

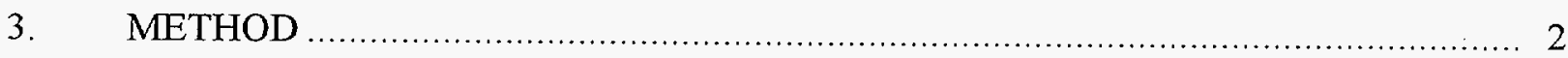

4. CODES, STANDARDS, AND REFERENCES ......................................... 3

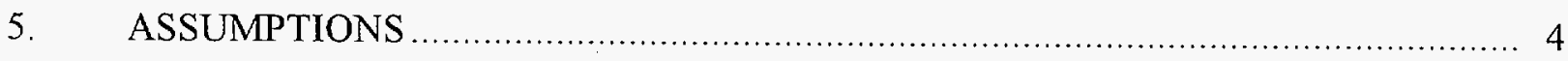

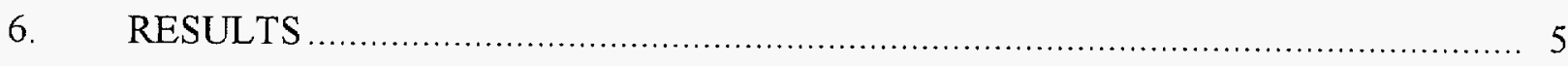

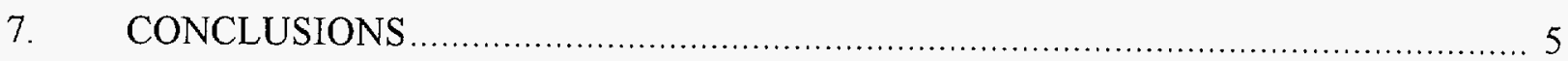

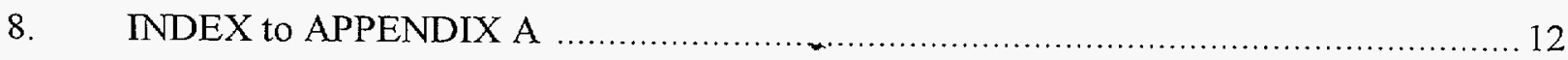

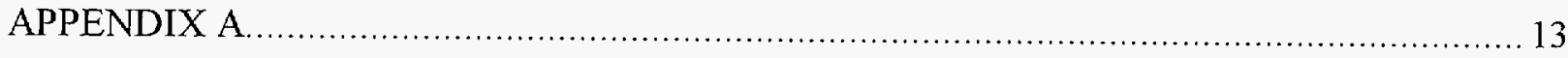

Appendix A Scenario Analysis Summaries and Mitigation Sheets .......................5010 to 5105.3 
DI: BAB000000-01717-0200-00004 Rev 02

\section{LIST OF TABLES}

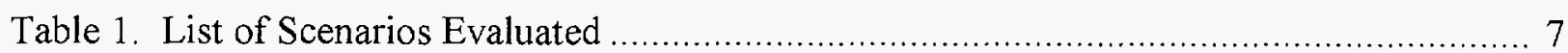


DI: BAB000000-01717-0200-00004 Rev 02

\section{LIST OF FIGURES}

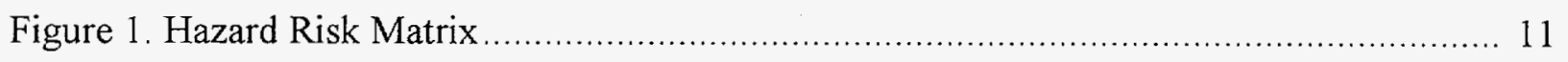


DI: BAB000000-01717-0200-00004 Rev 02

\section{WARNING}

ALTHOUGH EVERY EFFORT HAS GENERALLY BEEN MADE TO INCORPORATE SAFETY FEATURES INTO DESIGN, IT IS OFTEN NECESSARY TO RELY ON PROCEDURES AND TRAINING TO MITIGATE SITUATIONS THAT CAN PRODUCE HAZARDS. THEREFORE, SAFETY IS HEAVILY DEPENDENT ON ADEQUATE TRAINING AND ADHERANCE TO APPROVED PROCEDURES. INADEQUATE TRAINING OR FALURE TO STRICTLY ADHERE TO TRAINING AND FOLLOW APPROVED PROCEDURES CAN LEAD TO SEVERE INJURIES OR DEATH. 


\section{DI: BAB000000-01717-0200-00004 Rev 02}

\section{PURPOSE}

The purpose of this analysis is to systematically identify and evaluate hazards related to the design of the Yucca Mountain Project Exploratory Studies Facility (ESF) East-West Cross Drift. This analysis builds upon prior ESF System Safety Analyses and incorporates TS Main Drift scenarios, where applicable, into the East-West Drift scenarios. This System Safety Analysis (SSA) focuses on the personnel safety and health hazards associated with the engineered design of the East-West Drift. The analysis also evaluates other aspects of the East-West Drift, including purchased equipment (e.g., scientific mapping platform) or Systems/Structures/Components (SSCs) and out-of-tolerance conditions. In addition to recommending design mitigation features, the analysis identifies the potential need for procedures, training, or Job Safety Analyses (JSAs). The inclusion of this information in the SSA is intended to assist the organization(s) (e.g., constructor, Safety and Health, design) responsible for these aspects of the East-West Drift in evaluating personnel hazards and augment the information developed by these organizations.

The SSA is an integral part of the systems engineering process, whereby safety is considered during planning, design, testing, and construction. A largely qualitative approach is used which incorporates operating experiences and recommendations from vendors, the constructor and the operating contractor. The risk assessment in this analysis characterizes the scenarios associated with East-West Drift SSCs in terms of relative risk and includes recommendations for mitigating all identified hazards. The priority for recommending and implementing mitigation control features is:

1) Incorporate measures to reduce risks and hazards into SSC designs.

2) Add safety features and capabilities to existing designs.

3) Develop procedures and conduct training to increase worker awareness of potential hazards, reduce exposure to hazards, and inform personnel of the actions required to avoid accidents or correct hazardous conditions.

This analysis does not consider temporary construction items and, therefore, does not consider hazards associated with temporary construction items. This analysis will be reviewed and updated to reflect new East-West Drift design changes, construction modifications, and "as built" documentation of the East-West Drift when completed.

A major difference between this analysis and previous ESF SSAs is the inclusion of hazards that arise as a result of non-accident events, (e.g., "off-normal" operations, adverse environmental conditions, or "out-of-tolerance" conditions). Non-accident events, that were not included in previous ESF SSAs, include environmental and/or toxic hazards such as leaking gases/fluids, off-gassing reactions, and excessive dust, particulates, exhaust fumes, noise, temperature, etc. which could have an adverse health effect on personnel. 


\section{DI: BAB000000-01717-0200-00004 Rev 02}

\section{QUALITY ASSURANCE}

A QAP-2-0 evaluation was performed to determine whether System Safety Analyses are subject to QARD requirements. The results of this evaluation are presented in a QAP-2-0 Activity Evaluation for Specialty Engineering, dated August 7, 1997.

Based on the results of the QAP-2-0 evaluation, this analysis is not subject to requirements of the QARD.

\section{METHOD}

The System Safety Analysis process used in this analysis is described in YAP-30.48 (Reference 4.9) and is based on the methodology of MIL-STD-882C (Reference 4.5). The System Safety Analysis process is a systematic approach used to identify and mitigate system design-related hazards that could potentially have an adverse effect on personnel safety or health. The system safety analysis process includes four distinct steps: (1) hazard description (scenario development); (2) risk assignment (an initial risk rating) which is made prior to development of mitigations or controls; (3) development of hazard mitigations or controls; (4) risk designation (final risk rating) which considers that mitigations or controls are fully implemented.

Hazard description is the process of developing scenarios based upon the applicable hazards identified in a Preliminary Hazards Analysis (PHA). A Preliminary Hazards List (PHL), which is a result of the PHA, is generated by applying a generic checklist of potential internal and external events and phenomena to each SSC under consideration. The PHL is used as a direct input to the scenario development activity.

Risk assignment is an initial risk assessment that is performed before development of hazard mitigations or controls. It involves a qualitative frequency and consequence rating to determine a risk level for each scenario. The frequency rating is based on the estimated likelihood of a scenario occurring during the anticipated useful life of the affected SSC (useful life may vary for each SSC, depending on its intended function in the East-West Drift). The consequence rating is an estimate of the impact magnitude if the postulated scenario occurs. A qualitative level of risk (high, medium, low, extremely low) is determined for each scenario based on the frequency and consequence combinations using the Risk Rating Matrix found in the System Safety Analysis Procedure, YAP-30.48.

Hazard mitigation and control is the process by which specific measures are identified to prevent or mitigate potential hazards. Hazard mitigation and control can be applied prior to an event occurring or a condition/situation reaching a critical stage (i.e., "preventive") or after an event has occurred or a condition has exceeded acceptable limits (i.e., "postoccurrence"). In the first case (preventive), the mitigation and control features have a direct 


\section{DI: BAB000000-01717-0200-00004 Rev 02}

impact on the frequency; i.e., by reducing the probability of an event occurring or an acceptable limit being exceeded, the frequency rating can be lowered. In the second case (post occurrence), the mitigation and control features have a direct impact on the consequence; i.e., the event has occurred or the acceptable limit has been exceeded but the impact on personnel safety can be reduced or eliminated by implementing the mitigation and control features. Hazard mitigation is achieved through design selection, safety design features or devices, detection and warning devices, and/or the use of procedures and training.

Risk designation is a final risk rating that assumes that the mitigations or controls developed in the preceding activity have been fully implemented. The frequency and consequence ratings are reevaluated considering the mitigations and controls in order to determine a resulting level of risk (high, medium, low, extremely low). The Risk Rating Matrix from the System Safety Analysis procedure referenced above is utilized. The resulting or final risk designation level is scenario specific including the specific set of mitigation(s) developed in the analysis. The risk designation is not applicable to systems, processes or activities that are not controlled/designed per referenced codes and specifications, or which have mitigation features that are substantially different from those contained in the analysis.

The following SSCs were considered in this analysis:

- East-West Drift Excavation and Layout Design

- Linings and Ground Support

- Ventilation System

- 480V Power Line/Cables

- Compressed Air Line

- Electrical Switchgear

- Rail

- Rail Cars/Locomotives (e.g., man trains, muck transporters)

- Subsurface Vehicles (e.g., Alpine Miner, front-end loader, drill jumbo)

- Supply/Waste Water Line

- Subsurface Fire Protection System

- Engineering/Scientific Mapping Floor

The SSCs listed above were evaluated based upon current best-available design/functional information relative to the Starter Tunnel and East-West Drift. This analysis provided conservative estimates of the potential design safety hazards in cases where design/historical information was limited or unavailable.

\section{CODES, STANDARDS, AND REFERENCES}

4.1 DOE 1989. General Design Criteria. DOE Order 6430.1A. April 6, 1989. ACC: NNA.19900403.0021. TIC Catalog Number 219982. 


\section{DI: BAB000000-01717-0200-00004 Rev 02}

4.2 CRWMS M\&O 1995. Topopah Springs Main Drift System Safety Analysis. BAB000000-01717-0200-00149, REV 00. Las Vegas, Nevada: CRWMS M\&O. ACC: MOL.19960312.0256, MOL.19960513.00.

4.3 DOE 1992. Preliminary Safety Analysis Report for the Yucca Mountain Project Exploratory Studies Facility and Site Characterization Program. YMP/91-37, Rev. 0. DOE: OCRWM. ACC: NNA.19920831.0019, NNA. 19920831.0020

4.4 CRWMS M\&O 1997. Activity Evaluation: Specialty Engineering (1.2.1.8). Las Vegas, Nevada. August 7, 1997. CRWMS M\&O. ACC: MOL.19971215.0018.

4.5 DOD 1993. Military Standard System Safety Program Requirements. MILSTD-882C. January 19, 1993. TIC Catalog Number 209468.

4.6 DOE 1986. Safety Analysis and Review System. DOE Order 5481.1B. September 23, 1986. TIC Catalog Number 221740.

4.7 System Safety Society 1997. System Safety Analysis Handbook-A source book for safety practitioners. Second Edition. Albuquerque, New Mexico. System Safety Society. TIC Catalog Number 236411.

4.8 DOE 1995. System Safety Plan. YMP/94-13, Rev. 0. Las Vegas, Nevada: U.S. Department of Energy, Office of Civilian Radioactive Waste Management. ACC: MOL.19960130.0047.

4.9 DOE 1995. System Safety Analysis Procedure. YAP-30.48, Rev. 1, ICN 0. September 8, 1998. Las Vegas, Nevada. Yucca Mountain Site Characterization Office. ACC: MOL.19990125.0080.

4.10 CRWMS M\&O 1997. Enhanced Characterization of the Repository Block Requirements Document (TBV-227). BAB000000-01717-5705-00013 REV 0. June 13, 1997. Las Vegas, Nevada: CRWMS M\&O ACC:

MOL.19971103.0527.

4.11 OSHA 1990. Safety and Health Regulations for Construction. 29 CFR 1926. July 1, 1990. ACC: NNA.19910123.0044. TIC Catalog Number 238502.

\section{ASSUMPTIONS}




\section{DI: BAB000000-01717-0200-00004 Rev 02}

- A number of scenarios and SSCs (e.g., ventilation) in this analysis have counterparts in the Topopah Springs (TS) Main Drift System Safety Analysis. Because of the scarcity of detailed design information and the assumed similarity between the SSCs, SSC features, characteristics, and limitations identified for the TS Main Drift SSA were also applied for the conduct of the East-West Drift SSA.

- Standard SSCs common to the subsurface ESF (e.g., power/water/compressed air lines, electrical equipment) will be present in the East-West Drift.

- The Construction Management Organization (CMO) will verify that the mitigation features approved in this SSA are effectively implemented and provide implementation status to M\&O System Safety.

- The conclusions of this SSA pertain only to the scenarios identified herein.

- Future design changes will need to be evaluated to determine the impact on personnel safety and health.

- All procedures, training, manuals, and other documentation identified as mitigation features are complete, comprehensive, and accurate.

\section{RESULTS}

Due to the similarities of the designed systems in the TS Main Drift and the East-West Drift, the TS Main Drift scenarios were used as a basis for developing the East-West Drift scenarios. The TS Main Drift SSA and the design documentation for the East-West Drift were reviewed in detail. Specifically, this analysis focused on personnel safety and health hazards associated with the engineered design of the East-West Drift. The analysis also evaluated other aspects of the East-West Drift, including purchased equipment or SSCs and out-of-tolerance conditions.

The List of Scenarios Evaluated (Table 1) contains a brief description of each scenario in this analysis and a final frequency, consequence and risk rating that assumes all of the mitigations and controls recommended have been fully implemented. Forty-six (46) scenarios are listed. The detailed scenario analysis summaries are contained in Appendix A.

Each scenario was assigned a final risk level based upon the frequency and consequence of the potential hazards identified. In this analysis, there were zero (0) scenarios with a $\underline{\boldsymbol{h i g h}}$ risk designation, zero (0) scenarios with a medium risk designation, twenty-four (24) scenarios with a low risk designation, and twenty-two (22) scenarios with an extremely low 


\section{DI: BAB000000-01717-0200-00004 Rev 02}

risk designation. The Hazard Risk Matrix (Figure 1) illustrates the distribution of the scenarios risk ratings with in the matrix.

The risk designation serves two purposes:

1) It is a qualitative indicator that can be used by management to determine whether the level of risk is acceptable or if additional safety mitigation features must be implemented to lower the risk to an acceptable level.

2) It is a management tool that can be used to determine the order for addressing hazards and implementing the associated mitigation features.

\section{CONCLUSIONS}

The East-West Drift System Safety Analysis identified hazards related to the design and design/testing related construction of the ESF East-West Drift. The consequences of the hazards were analyzed, mitigation measures to control the hazards by design and/or administrative controls were identified, and an assessment of the risk(s) was performed. Information concerning the design of the East-West Drift was obtained from the M\&O ESF Design organization; discussions with the designers; and the exchange of information between DOE, M\&O and Kiewit personnel during the SSA development and review process. The final risk assessments were the subject of formal detailed reviews by a System Safety Working Group and recognized as credible hazard scenarios for the East-West Drift. 
DI: BAB000000-01717-0200-00004 Rev 02

Table 1 - List of Scenarios Evaluated

\begin{tabular}{|c|c|c|c|c|}
\hline $\begin{array}{l}\text { Scenario ID } \\
\text { Number }\end{array}$ & Risk Level & Frequency & Consequence & Scenario Description \\
\hline $\begin{array}{l}\text { UE5088 } \\
5 \text { Mitigation } \\
\text { Sheets }\end{array}$ & Low & E - & I - & $\begin{array}{l}\text { Personnel injury/equipment damage caused by failure of } \\
\text { ground support due to earthquake. }\end{array}$ \\
\hline $\begin{array}{l}\text { UI5010 } \\
7 \text { Mitigation } \\
\text { Sheets }\end{array}$ & Low & $\begin{array}{l}\text { E - } \\
\text { Improbable }\end{array}$ & $\begin{array}{l}\text { I - } \\
\text { Catastrophic }\end{array}$ & $\begin{array}{l}\text { Accident due to load shifting or equipment/materials } \\
\text { protruding from a subsurface transport vehicle (e.g., } \\
\text { mantrip, flat car, utility car). }\end{array}$ \\
\hline $\begin{array}{l}\text { UI5011 } \\
8 \text { Mitigation } \\
\text { Sheets }\end{array}$ & Low & $\begin{array}{l}\text { E - } \\
\text { Improbable }\end{array}$ & $\begin{array}{l}\text { I - } \\
\text { Catastrophic }\end{array}$ & $\begin{array}{l}\text { Subsurface vehicle (e.g., Alpine Miner, front-end loader, } \\
\text { drill jumbo) accident. }\end{array}$ \\
\hline $\begin{array}{l}\text { UI5017 } \\
12 \text { Mitigation } \\
\text { Sheets }\end{array}$ & $\begin{array}{l}\text { Extremely } \\
\text { Low }\end{array}$ & $\begin{array}{l}\text { E - } \\
\text { Improbable }\end{array}$ & III - Marginal & $\begin{array}{l}\text { Ventilation system failure or out-of-tolerance condition } \\
\text { results in personnel health hazard (see also UI5060 and } \\
\text { U15042). }\end{array}$ \\
\hline $\begin{array}{l}\text { UI5025 } \\
4 \text { Mitigation } \\
\text { Sheets }\end{array}$ & $\begin{array}{l}\text { Extremely } \\
\text { Low }\end{array}$ & $\begin{array}{l}\text { E - } \\
\text { Improbable }\end{array}$ & III - Marginal & Compressed air line rupture results in personnel injury. \\
\hline $\begin{array}{l}\text { UI5028 } \\
10 \text { Mitigation } \\
\text { Sheets }\end{array}$ & $\begin{array}{l}\text { Extremely } \\
\text { Low }\end{array}$ & $\begin{array}{l}\text { E - } \\
\text { Improbable }\end{array}$ & III - Marginal & $\begin{array}{l}\text { Fire involving subsurface vehicle (e.g., Alpine Miner, } \\
\text { front-end loader, drill jumbo) or temporarily stored } \\
\text { materials. }\end{array}$ \\
\hline $\begin{array}{l}\text { UI5030 } \\
8 \text { Mitigation } \\
\text { Sheets }\end{array}$ & $\begin{array}{l}\text { Extremely } \\
\text { Low }\end{array}$ & D - Remote & III - Marginal & $\begin{array}{l}\text { Fire due to failure/short/arc/overload of electrical } \\
\text { switchgear or other electrical equipment (e.g., } \\
\text { transformer, electrical panel/box, electrical wire or } \\
\text { cable). }\end{array}$ \\
\hline $\begin{array}{l}\text { UI5042 } \\
6 \text { Mitigation } \\
\text { Sheets }\end{array}$ & $\begin{array}{l}\text { Extremely } \\
\text { Low }\end{array}$ & D - Remote & III - Marginal & $\begin{array}{l}\text { Air Quality - Toxic fumes (e.g., carbon monoxide, } \\
\text { carbon dioxide, nitrogen dioxide, NOx) from diesel } \\
\text { exhaust results in a personnel health hazard (see also } \\
\text { U15070). }\end{array}$ \\
\hline $\begin{array}{l}\text { UI5046 } \\
1 \text { Mitigation } \\
\text { Sheet }\end{array}$ & Low & B - Probable & $\begin{array}{l}\text { IV - } \\
\text { Negligible }\end{array}$ & $\begin{array}{l}\text { Train/locomotive derailment at turnout (intersection of } \\
\text { East-West Starter Tunnel and TS Main Drift). }\end{array}$ \\
\hline $\begin{array}{l}\text { UI5047 } \\
6 \text { Mitigation } \\
\text { Sheets }\end{array}$ & Low & A - Frequent & $\begin{array}{l}\text { IV - } \\
\text { Negligible }\end{array}$ & $\begin{array}{l}\text { General slips and trips resulting in minor personnel } \\
\text { injury (see also U15069). }\end{array}$ \\
\hline $\begin{array}{l}\text { UI5048 } \\
5 \text { Mitigation } \\
\text { Sheets }\end{array}$ & $\begin{array}{l}\text { Extremely } \\
\text { Low }\end{array}$ & D - Remote & III - Marginal & $\begin{array}{l}\text { Personnel injury due to tools, equipment, or debris } \\
\text { dropping or falling from an elevated platform. }\end{array}$ \\
\hline $\begin{array}{l}\text { UI5050 } \\
8 \text { Mitigation } \\
\text { Sheets }\end{array}$ & Low & $\begin{array}{l}\text { E - } \\
\text { Improbable }\end{array}$ & $\begin{array}{l}\text { I - } \\
\text { Catastrophic }\end{array}$ & $\begin{array}{l}\text { Personnel injury/equipment damage due to runaway } \\
\text { vehicle (e.g., train, locomotive, front-end loader, drill } \\
\text { jumbo) on slope. }\end{array}$ \\
\hline $\begin{array}{l}\text { UI5054 } \\
8 \text { Mitigation } \\
\text { Sheets }\end{array}$ & $\begin{array}{l}\text { Extremely } \\
\text { Low }\end{array}$ & D - Remote & III - Marginal & $\begin{array}{l}\text { Personnel injury due to failure of ground support (e.g., } \\
\text { rock fall). }\end{array}$ \\
\hline
\end{tabular}




\section{DI: BAB000000-01717-0200-00004 Rev 02}

Table 1 - List of Scenarios Evaluated

\begin{tabular}{|c|c|c|c|c|}
\hline $\begin{array}{l}\text { Scenario ID } \\
\text { Number }\end{array}$ & Risk Level & Frequency & Consequence & Scenario Description \\
\hline $\begin{array}{l}\text { UI5057 } \\
12 \text { Mitigation } \\
\text { Sheets }\end{array}$ & $\begin{array}{l}\text { Extremely } \\
\text { Low }\end{array}$ & D - Remote & III - Marginal & $\begin{array}{l}\text { General underground fire due to ignition of trash, } \\
\text { solvents, chemicals, or other combustible product. }\end{array}$ \\
\hline $\begin{array}{l}\text { UI5058 } \\
5 \text { Mitigation } \\
\text { Sheets }\end{array}$ & Low & $\begin{array}{l}\text { E - } \\
\text { Improbable }\end{array}$ & II - Critical & Electrical shock resulting in personnel injury. \\
\hline $\begin{array}{l}\text { UI5060 } \\
6 \text { Mitigation } \\
\text { Sheets }\end{array}$ & Low & B - Probable & $\begin{array}{l}\text { IV - } \\
\text { Negligible }\end{array}$ & $\begin{array}{l}\text { Air Quality - Excessive dust results in a personnel health } \\
\text { hazard (see also UI5017 and UI5070). }\end{array}$ \\
\hline $\begin{array}{l}\text { UI5061 } \\
5 \text { Mitigation } \\
\text { Sheets }\end{array}$ & $\begin{array}{l}\text { Extremely } \\
\text { Low }\end{array}$ & $\begin{array}{l}\mathrm{C}- \\
\text { Occasional }\end{array}$ & $\begin{array}{l}\text { IV - } \\
\text { Negligible }\end{array}$ & $\begin{array}{l}\text { Excessive noise due to Alpine Miner, rock bolt drills, } \\
\text { ventilation fans, drilling/blasting, or other potential } \\
\text { sources of noise. }\end{array}$ \\
\hline $\begin{array}{l}\text { UI5062 } \\
5 \text { Mitigation } \\
\text { Sheets }\end{array}$ & Low & $\begin{array}{l}\text { E - } \\
\text { Improbable }\end{array}$ & II - Critical & Worker injury due to fall from an elevated platform. \\
\hline $\begin{array}{l}\text { UI5067 } \\
6 \text { Mitigation } \\
\text { Sheets }\end{array}$ & $\begin{array}{l}\text { Extremely } \\
\text { Low }\end{array}$ & D - Remote & III - Marginal & $\begin{array}{l}\text { Subsurface vehicle (e.g., Alpine Miner, front-end loader, } \\
\text { drill jumbo) or machinery collision with utility cable, } \\
\text { conduit or pipe (e.g., ventilation, water supply, } \\
\text { subsurface waste water, compressed air, electrical line, } \\
\text { communications line, data line). }\end{array}$ \\
\hline $\begin{array}{l}\text { UI5069 } \\
6 \text { Mitigation } \\
\text { Sheets }\end{array}$ & Low & $\begin{array}{l}\text { E - } \\
\text { Improbable }\end{array}$ & $\begin{array}{l}\text { I - } \\
\text { Catastrophic }\end{array}$ & $\begin{array}{l}\text { General slips and trips resulting in severe personnel } \\
\text { injury (see also UI5047). }\end{array}$ \\
\hline $\begin{array}{l}\text { UI5070 } \\
5 \text { Mitigation } \\
\text { Sheets }\end{array}$ & $\begin{array}{l}\text { Extremely } \\
\text { Low }\end{array}$ & D - Remote & III - Marginal & $\begin{array}{l}\text { Air Quality - Excessive diesel soot and/or smoke } \\
\text { particulates results in a personnel health hazard (see also } \\
\text { UI5042). }\end{array}$ \\
\hline $\begin{array}{l}\text { UI5072 } \\
4 \text { Mitigation } \\
\text { Sheets }\end{array}$ & Low & $\begin{array}{l}\text { E - } \\
\text { Improbable }\end{array}$ & II - Critical & Ventilation fan structural failure. \\
\hline $\begin{array}{l}\text { UI5073 } \\
6 \text { Mitigation } \\
\text { Sheets }\end{array}$ & $\begin{array}{l}\text { Extremely } \\
\text { Low }\end{array}$ & D - Remote & III - Marginal & $\begin{array}{l}\text { Personnel injury/equipment damage due to muck falling } \\
\text { off the conveyor system. }\end{array}$ \\
\hline $\begin{array}{l}\text { UI5075 } \\
4 \text { Mitigation } \\
\text { Sheets }\end{array}$ & Low & $\begin{array}{l}\text { E - } \\
\text { Improbable }\end{array}$ & II - Critical & $\begin{array}{l}\text { Personnel injury resulting from misuse of rail cars for } \\
\text { personnel transit. }\end{array}$ \\
\hline $\begin{array}{l}\text { UI5077 } \\
4 \text { Mitigation } \\
\text { Sheets }\end{array}$ & Low & $\begin{array}{l}\text { E - } \\
\text { Improbable }\end{array}$ & $\begin{array}{l}\text { I - } \\
\text { Catastrophic }\end{array}$ & $\begin{array}{l}\text { Personnel injury/equipment damage due to falling or } \\
\text { partially detached hanging installed equipment (e.g., } \\
\text { ventilation ducts, conveyor support framing, power lines, } \\
\text { service lines). }\end{array}$ \\
\hline $\begin{array}{l}\text { U15078 } \\
2 \text { Mitigation } \\
\text { Sheets }\end{array}$ & Low & B - Probable & $\begin{array}{l}\text { IV - } \\
\text { Negligible }\end{array}$ & Ventilation fan failure (foreign object(s) in system). \\
\hline $\begin{array}{l}\text { UI5080 } \\
4 \text { Mitigation } \\
\text { Sheets }\end{array}$ & Low & $\begin{array}{l}\text { E - } \\
\text { Improbable }\end{array}$ & II - Critical & Fire hazards: Conveyor system. \\
\hline
\end{tabular}


DI: BAB000000-01717-0200-00004 Rev 02

Table 1 - List of Scenarios Evaluated

\begin{tabular}{|c|c|c|c|c|}
\hline $\begin{array}{l}\text { Scenario ID } \\
\text { Number }\end{array}$ & Risk Level & Frequency & Consequence & Scenario Description \\
\hline $\begin{array}{l}\text { U15081 } \\
5 \text { Mitigation } \\
\text { Sheets }\end{array}$ & $\begin{array}{l}\text { Extremely } \\
\text { Low }\end{array}$ & E - & $\begin{array}{l}\text { IV - } \\
\text { Negligible }\end{array}$ & Fire hazards: Ventilation system. \\
\hline $\begin{array}{l}\text { UI5082 } \\
2 \text { Mitigation } \\
\text { Sheets }\end{array}$ & Low & B - Probable & $\begin{array}{l}\text { IV - } \\
\text { Negligible }\end{array}$ & Ventilation system shutdown due to main power failure. \\
\hline $\begin{array}{l}\text { UI5083 } \\
4 \text { Mitigation } \\
\text { Sheets }\end{array}$ & $\begin{array}{l}\text { Extremely } \\
\text { Low }\end{array}$ & $\begin{array}{l}\text { E - } \\
\text { Improbable }\end{array}$ & $\begin{array}{l}\text { IV - } \\
\text { Negligible }\end{array}$ & Lighting system failure for an extended period of time. \\
\hline $\begin{array}{l}\text { UT5084 } \\
3 \text { Mitigation } \\
\text { Sheets }\end{array}$ & $\begin{array}{l}\text { Extremely } \\
\text { Low }\end{array}$ & D - Remote & III - Marginal & $\begin{array}{l}\text { Diesel fuel hazard: Leak in engine compartment provides } \\
\text { fuel source for possible fire. }\end{array}$ \\
\hline $\begin{array}{l}\text { UI5085 } \\
10 \text { Mitigation } \\
\text { Sheets }\end{array}$ & Low & $\begin{array}{l}\text { E - } \\
\text { Improbable }\end{array}$ & $\begin{array}{l}\text { I - } \\
\text { Catastrophic }\end{array}$ & Vehicle hits personnel on foot in the East-West Drift. \\
\hline $\begin{array}{l}\text { UI5086 } \\
5 \text { Mitigation } \\
\text { Sheets }\end{array}$ & Low & $\begin{array}{l}\text { E - } \\
\text { Improbable }\end{array}$ & II - Critical & $\begin{array}{l}\text { Vehicle diesel fire hazard: Diesel leak(s) from fuel tank } \\
\text { or fuel lines or spills during refueling. }\end{array}$ \\
\hline $\begin{array}{l}\text { UI5087 } \\
8 \text { Mitigation } \\
\text { Sheets }\end{array}$ & Low & $\begin{array}{l}\text { E - } \\
\text { Improbable }\end{array}$ & $\begin{array}{l}\text { I - } \\
\text { Catastrophic }\end{array}$ & $\begin{array}{l}\text { Train/locomotive derailment and/or impacting the TBM } \\
\text { causing personnel injury and/or major equipment } \\
\text { damage. }\end{array}$ \\
\hline $\begin{array}{l}\text { U15089 } \\
6 \text { Mitigation } \\
\text { Sheets }\end{array}$ & Low & $\begin{array}{l}\text { E - } \\
\text { Improbable }\end{array}$ & II - Critical & $\begin{array}{l}\text { Personnel working on the mapping floor come into } \\
\text { contact with operating much conveyor system } \\
\text { mechanical parts. }\end{array}$ \\
\hline $\begin{array}{l}\text { UI5091 } \\
3 \text { Mitigation } \\
\text { Sheets }\end{array}$ & $\begin{array}{l}\text { Extremely } \\
\text { Low }\end{array}$ & $\begin{array}{l}\mathrm{C}- \\
\text { Occasional }\end{array}$ & $\begin{array}{l}\text { IV - } \\
\text { Negligible }\end{array}$ & $\begin{array}{l}\text { Mapping floor door(s) fall into path of the train or } \\
\text { against moving train or cars. }\end{array}$ \\
\hline $\begin{array}{l}\text { U15092 } \\
6 \text { Mitigation } \\
\text { Sheets }\end{array}$ & $\begin{array}{l}\text { Extremely } \\
\text { Low }\end{array}$ & D - Remote & $\begin{array}{l}\text { IV - } \\
\text { Negligible }\end{array}$ & $\begin{array}{l}\text { Train strikes equipment or mapping floor doors while } \\
\text { transiting the mapping floor. }\end{array}$ \\
\hline $\begin{array}{l}\text { UI5093 } \\
8 \text { Mitigation } \\
\text { Sheets }\end{array}$ & Low & $\begin{array}{l}\text { E - } \\
\text { Improbable }\end{array}$ & II - Critical & $\begin{array}{l}\text { Train strikes personnel while transiting the mapping } \\
\text { floor. }\end{array}$ \\
\hline $\begin{array}{l}\text { UI5094 } \\
6 \text { Mitigation } \\
\text { Sheets }\end{array}$ & Low & B - Probable & $\begin{array}{l}\text { IV - } \\
\text { Negligible }\end{array}$ & $\begin{array}{l}\text { Minor personnel injuries due to falls/trips/slips on the } \\
\text { mapping floor. }\end{array}$ \\
\hline $\begin{array}{l}\text { UI5096 } \\
1 \text { Mitigation } \\
\text { Sheet }\end{array}$ & Low & $\begin{array}{l}\text { E - } \\
\text { Improbable }\end{array}$ & $\begin{array}{l}\text { I - } \\
\text { Catastrophic }\end{array}$ & $\begin{array}{l}\text { Personnel injury due to crawling or becoming stuck } \\
\text { under the transition ramp and/or mapping floor to } \\
\text { retrieve items. }\end{array}$ \\
\hline $\begin{array}{l}\text { UI5097 } \\
2 \text { Mitigation } \\
\text { Sheets }\end{array}$ & $\begin{array}{l}\text { Extremely } \\
\text { Low }\end{array}$ & D - Remote & III - Marginal & $\begin{array}{l}\text { Personnel injury (pinch, amputation, crush) from } \\
\text { opening/closing mapping floor doors or the access panels } \\
\text { to the pressurized air system valves (located in the rail } \\
\text { decks) }\end{array}$ \\
\hline $\begin{array}{l}\text { UI5098 } \\
1 \text { Mitigation } \\
\text { Sheet }\end{array}$ & $\begin{array}{l}\text { Extremely } \\
\text { Low }\end{array}$ & D - Remote & III - Marginal & $\begin{array}{l}\text { If the air winches (tuggers) are ever used to move the } \\
\text { mapping floor along the tracks to a new location and a } \\
\text { cable fails or slips resulting in personnel injury. }\end{array}$ \\
\hline
\end{tabular}


DI: BAB000000-01717-0200-00004 Rev 02

Table 1 - List of Scenarios Evaluated

\begin{tabular}{|c|c|c|c|c|}
\hline $\begin{array}{l}\text { Scenario ID } \\
\text { Number }\end{array}$ & Risk Level & Frequency & Consequence & Scenario Description \\
\hline $\begin{array}{l}\text { U15099 } \\
3 \text { Mitigation } \\
\text { Sheets }\end{array}$ & Low & $\begin{array}{l}\text { E- } \\
\text { Improbable }\end{array}$ & II - Critical & $\begin{array}{l}\text { Tow cable slips or breaks and the train operator loses } \\
\text { positive control of the raised/moving mapping floor. }\end{array}$ \\
\hline $\begin{array}{l}\text { UI5100 } \\
1 \text { Mitigation } \\
\text { Sheet }\end{array}$ & $\begin{array}{l}\text { Extremely } \\
\text { Low }\end{array}$ & D - Remote & III - Marginal & $\begin{array}{l}\text { Personnel are injured while riding on the elevated } \\
\text { mapping cars during movement. }\end{array}$ \\
\hline $\begin{array}{l}\text { UI5103 } \\
3 \text { Mitigation } \\
\text { Sheets }\end{array}$ & $\begin{array}{l}\text { Extremely } \\
\text { Low }\end{array}$ & $\begin{array}{l}\text { E - } \\
\text { Improbable }\end{array}$ & III - Marginal & $\begin{array}{l}\text { Personnel receive electrical shock(s) from defective } \\
\text { electrical equipment that shorts/burns out or from } \\
\text { severed connectors. }\end{array}$ \\
\hline $\begin{array}{l}\text { U15105 } \\
3 \text { Mitigation } \\
\text { Sheets }\end{array}$ & $\begin{array}{l}\text { Extremely } \\
\text { Low }\end{array}$ & D - Remote & III - Marginal & $\begin{array}{l}\text { Installed electrical outlets/access panels/electrical } \\
\text { connectors/cables are damaged when the mapping floor } \\
\text { is moved. }\end{array}$ \\
\hline
\end{tabular}




\section{DI: BAB000000-01717-0200-00004 Rev 02}

HAZARD RISK MATRIX

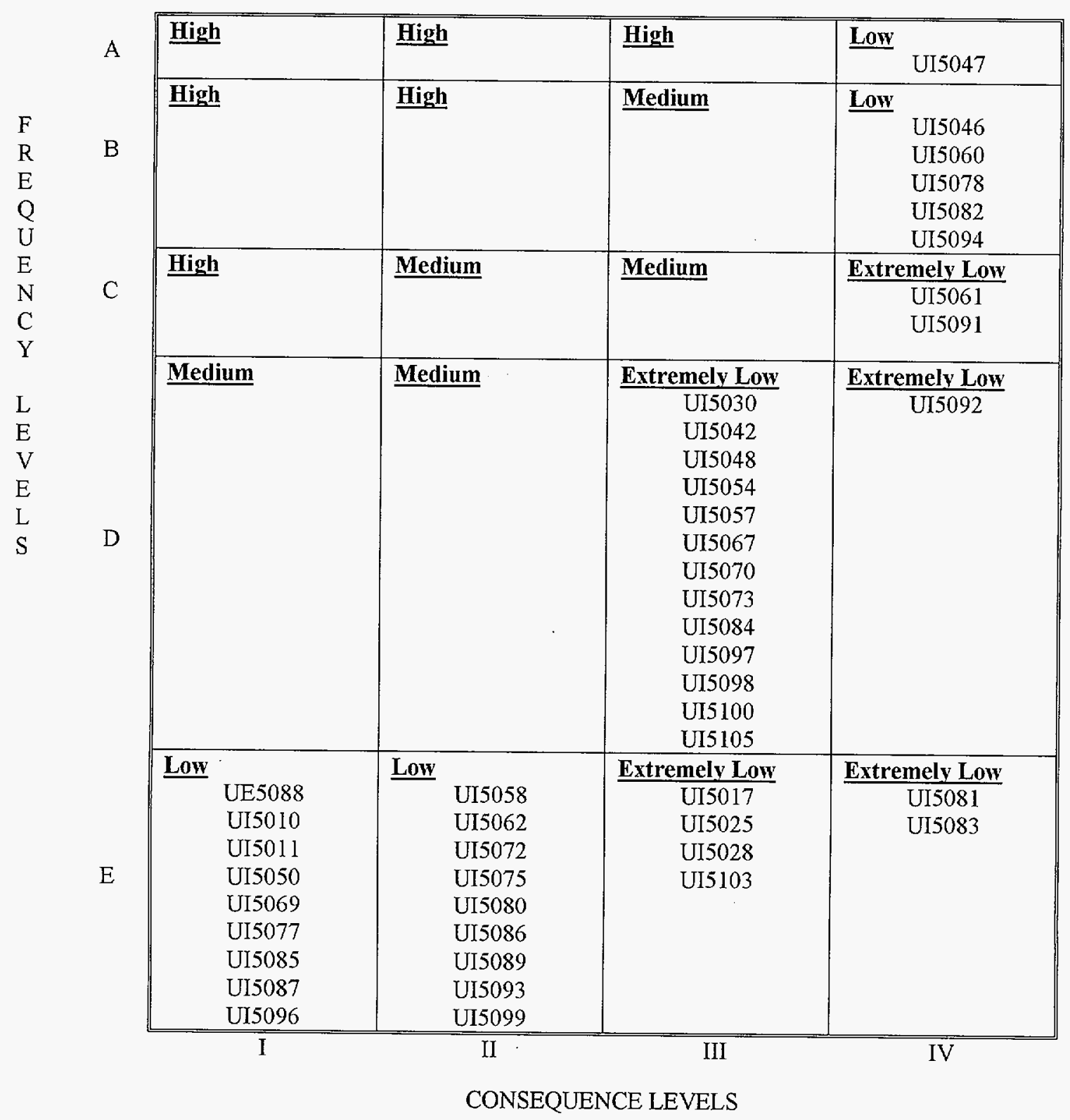

Figure 1. Scenarios Distributed Over Risk Matrix

Frequency Levels

A - Frequent

B - Probable

C - Occasional

D - Remote

E - Improbable
Consequence Levels

I - Catastrophic

II - Critical

III - Marginal

IV - Negligible 
DI: BAB000000-01717-0200-00004 Rev 02

8. INDEX TO APPENDIX A

Scenario Analysis Summaries and Mitigations

\begin{tabular}{|c|c|}
\hline Scenario & Scenario/Mitigation Pages \\
\hline UE5088 & UE5088 to UE5088.5 \\
\hline UI5010 & UI5010 to U15010.7 \\
\hline UI5011 & UI5011 to UI5011.8 \\
\hline UI5017 & UI5017 to UI5017.12 \\
\hline UI5025 & UI5025 to UI5025.4 \\
\hline UI5028 & UI5028 to UI5028.10 \\
\hline UI5030 & UI5030 to UI5030.8 \\
\hline UI5042 & UI5042 to UI5042.6 \\
\hline UI5046 & UI5046 to UI5046. 1 \\
\hline UI5047 & UI5047 to UI5047.6 \\
\hline UI5048 & UI5048 to UI5048.5 \\
\hline UI5050 & UI5050 to UI5050.8 \\
\hline UI5054 & UI5054 to UI5054.8 \\
\hline UI5057 & UI5057 to UI5057.12 \\
\hline UI5058 & UI5058 to UI5058.5 \\
\hline UI5060 & UI5060 to UI5060.6 \\
\hline UI5061 & UI5061 to UI5061.5 \\
\hline UI5062 & UI5062 to UI5062.5 \\
\hline UI5067 & UI5067 to UI5067.6 \\
\hline UI5069 & UI5069 to UI5069.6 \\
\hline UI5070 & UI5070 to UI5070.5 \\
\hline UI5072 & UI5072 to UI5072.4 \\
\hline UI5073 & UI5073 to UI5073.6 \\
\hline UI5075 & UI5075 to UI5075.4 \\
\hline UI5077 & UI5077 to UI5077.4 \\
\hline UI5078 & U15078 to UI5078.2 \\
\hline UI5080 & UI5080 to UI5080.4 \\
\hline UI5081 & UI5081 to UI5081.5 \\
\hline UI5082 & UI5082 to UI5082.2 \\
\hline UI5083 & UI5083 to UI5083.4 \\
\hline UI5084 & UI5084 to UI5084.3 \\
\hline UI5085 & UI5085 to UI5085.10 \\
\hline UI5086 & UI5086 to UI5086.5 \\
\hline UI5087 & UI5087 to UI5087.8 \\
\hline UI5089 & UI5089 to UI5089.6 \\
\hline UI5091 & UI5091 to UI5091.3 \\
\hline UI5092 & UI5092 to UI5092.6 \\
\hline UI5093 & UI5093 to UI5093.8 \\
\hline UI5094 & UI5094 to UI5094.6 \\
\hline UI5096 & UI5096 to UI5096. 1 \\
\hline UI5097 & UI5097 to UI5097.2 \\
\hline UI5098 & UI5098 to UI5098.1 \\
\hline UI5099 & UI5099 to UI5099.3 \\
\hline UI5100 & UI5 100 to UI5 100.1 \\
\hline UI5103 & UI5 103 to UI5 103.3 \\
\hline UI5105 & UI5 105 to UI5 105.3 \\
\hline
\end{tabular}


DI: BAB000000-01717-0200-00004 Rev 02

APPENDIX A 


\section{Scenario Description and Analysis}

\section{Approved}

Scenario Number:

Revision Number:

Revision Date:

Scenario:

Cause, Failure, or Hazardous Event:

Risk Assignment

Before Considering Mitigations:

After

Applying

Mitigations:

Prevention

Mitigation

Documentation:

\begin{tabular}{|l|}
\hline UE5088 \\
\hline 02 \\
\hline $12 / 17 / 98$ \\
\hline
\end{tabular}

$\begin{aligned} & \text { SSA } \text { East-West Drift } \\ & \text { Location: East-West Drift }\end{aligned}$

Personnel injury/equipment damage caused by failure of ground support due to earthquake.

Earthquake induced failure of ground support system components (steel sets, rockbolts).

Earthquake induced failure of ground support system components (steel sets, rockbolts).

\begin{tabular}{|lll|}
\hline Frequency Rating: & Consequence Rating: & *Risk Before: \\
A - Frequent & I-Catastrophic & \\
B - Probable & II - Critical & Medium \\
C - Occasional & III - Marginal & \\
D - Remote & $\bigcirc$ IV - Negligible & \\
E- Improbable & & \\
\hline
\end{tabular}

\begin{tabular}{|lll|}
\hline Frequency Rating: & Consequence Rating: & Risk After: \\
A - Frequent & I- Catastrophic & \\
B - Probable & $\bigcirc I I$ - Critical & Low \\
C - Occasional & $\bigcirc$ III - Marginal & \\
D - Remote & $\bigcirc$ IV - Negligible & \\
E - Improbable & & \\
\hline
\end{tabular}

UE0361, UE0362

Test, Inspection and Material Dedication Analysis: Shotcrete, Rockbolt and Accessories, BABEE0000-01717-0200-00006 Rev. O0B

ESF Ground Support Design Analysis, BABEEO000-01717-0200-00002 Rev. O0C OSHA - 29 CFR 1926

Emergency evacuation plan and procedures.

Training and safety manuals. 
DI: BAB000000-01717-0200-0004; Rev 02

\section{Mitigation Feature}

Scenario Number: UE5088 Mitigation Number: 1

Scenario: $\quad$ Personnel injury/equipment damage caused by failure of ground support due to earthquake.

Mitigation Feature: Install steel sets, lagging, tockbolts, wire mesh and shotcrete based on analysis of ground type and proximity to faults.

\section{Documentation:}

Remarks:

Mitigation type:

Design Feature

Safety Device

Warning Device

Procedure

$O$ Training

\section{Mitigation Tracking}

\section{Mitigation Implementation Status:}

Comments:
Not Implemented

Implemented

Implemented (per T.Rotert, aug98_Ground Support is installed as warranted) wp.

A\&E ground support walkdowns are providing analysis and recommendations and remedial ground support. Per Bob Law 5/5/99, JR 


\section{DI: BAB000000-01717-0200-0004, Rev 02}

\section{Mitigation Feature}

Scenario Number: UE5088 Mitigation Number: 2

Scenario:

Personnel injury/equipment damage caused by failure of ground support due to earthquake.

Mitigation Feature:

Establish installation inspection/acceptance procedures for steel sets, rockbolts, wire mesh and shotcrete.

Documentation:

Remarks:

Mitigation type:
Design Feature
Safety Device
Warning Device
Procedure
Training

\section{Mitigation Tracking}

Mitigation Implementation Status:

Comments:
Not Implemented

Implemented

Implemented (per T. Rotert, aug98) wp.

\section{Appendix A}

Page UE5088.2 


\section{DI: BAB000000-01717-0200-0004, Rev 02}

\section{Mitigation Feature}

Scenario Number:

\begin{tabular}{|l|l|}
\hline UE5088 & Mitigation Number: 3 \\
\hline
\end{tabular}

Scenario:

Personnel injury/equipment damage caused by failure of ground support due to earthquake.

Mitigation Feature: Visually inspect the ground support system on a weekly basis and prior to reoccupation following any earthquake event.

Documentation:

Remarks:

Mitigation type:
Design Feature
$\bigcirc$ Safety Device
Warning Device
Procedure
Training

\section{Mitigation Tracking}

\section{Mitigation Implementation Status:}

Comments:
Not Implemented

Implemented

Implemented (per T. Rotert, aug98 Weekly inspections by A/E and supervisors.) wp.

Appendix A

Page UE5088.3 


\section{DI: BAB000000-01717-0200-0004; Rev 02}

\section{Mitigation Feature}

$\begin{array}{ll}\text { Scenario Number: } & \text { UE50s8 } \\ \text { Scenario: } & \text { Mersonnel injuryfequipment damage caused by failure of ground support due to earthquake. } \\ \text { Mitigation Feature: } & \begin{array}{l}\text { Develop procedures for cessation of work and evacuation of personnel following earthquake } \\ \text { event. }\end{array} \\ \text { Documentation: } & \\ \text { Remarks: } & \\ \text { Mitigation type: } & \text { Design Feature } \\ & \text { Safety Device } \\ \text { Warning Device } & \text { Procedure } \\ \text { Training }\end{array}$

\section{Mitigation Tracking}

Mitigation Implementation Status:

Comments:
Not Implemented

Implemented

Appendix A

Page UE5088.4 
DI: BAB000000-01717-0200-0004, Rev 02

\section{Mitigation Feature}

Scenario Number:

UE5088 Mitigation Number:

5

Scenario: $\quad$ Personnel injury/equipment damage caused by failure of ground support due to earthquake.

Mitigation Feature: Safety training in evacuation procedures.

Documentation:

Remarks:

Mitigation type:
Design Feature
Safety Device
Warning Device
Procedure
Training

\section{Mitigation Tracking}

Mitigation Implementation

Status:

Comments:
Not Implemented
implemented

Implemented (per T. Rotert, aug98 GUT training covers evacuation procedure) wp. 


\section{Scenario Description and Analysis}

\section{Approved}

Scenario Number: Revision Number: Revision Date:

Scenario:

Cause, Failure, or Hazardous Event:

\begin{tabular}{|l|}
\hline$U 15010$ \\
\hline 02 \\
\hline $12 / 17 / 98$ \\
\hline
\end{tabular}

Accident due to load shifting or equipment/materials protruding from a subsurface transport vehicle (e.g., mantrip, flat car, utility car).

Brake failure; load shifting; lack of proper equipment/material restraints/guards; failure to adhere to safety and operating procedures.
Risk Assignment Before Considering Mitigations:

\begin{tabular}{|ll}
\hline Frequency Rating: & Consequence Rating: \\
A - Frequent & I - Catastrophic \\
B-Probable & II - Critical \\
C- Occasional & III - Marginal \\
D-Remote & IV - Negligible \\
E- Improbable &
\end{tabular}

Risk Designation

After

Applying

Mitigations:

\section{*Risk Before:}

High
Consequence Rating:
I - Catastrophic
II - Critical
OII - Marginal
OIV - Negligible

Low

E - Improbable

Related Scenarios:

U10021, U10022, U10025

29 CFR 1926.800; training manuals; safety manuals; JSAs.

Prevention

Mitigation

Documentation:

\section{Appendix A \\ Page UI5010}




\section{DI: BAB000000-01717-0200-0004, Rev 02}

\section{Mitigation Feature}

Scenario Number: $415010 \quad$ Mitigation Number: 1

Scenario:

Accident due to load shifting or equipment/materials protruding from a subsurface transport vehicle (e.g., mantrip, flat car, utility car).

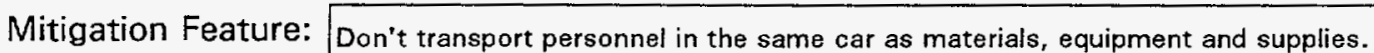

Documentation: 29 CFR 1926.800; training manuals; safety manuals; JSAs.

Remarks:

Mitigation type: $\quad$\begin{tabular}{|l}
$\bigcirc$ Design Feature \\
$\bigcirc$ Safety Device \\
$\bigcirc$ Warning Device \\
$\bigcirc$ Procedure \\
Training
\end{tabular}

\section{Mitigation Tracking}

Mitigation Implementation

Status:

Comments:

\section{Not Implemented}

Implemented

Implemented (per T. Rotert, aug98_Materials, supplies and equipment are transported separately) wp. 


\section{DI: BAB000000-01717-0200-0004, Rev 02}

\section{Mitigation Feature}

Scenario Number: U15010 Mitigation Number:

Scenario: $\quad$ Accident due to load shifting or equipment/materials protruding from a subsurface transport vehicle (e.g., mantrip, flat car, utility car).

Mitigation Feature: Design/select cars with adequate geometry and capacity to handle load weights and shifting of load.

Documentation:

Remarks:

Mitigation type:

Design Feature

Safety Device

Warning Device

Procedure

Training

\section{Mitigation Tracking}

Mitigation

Implementation

Status:

Not Implemented

Implemented

Comments: $\quad$ Implemented (per T. Rotert, aug98) wp.

Appendix A

Page UI5010.2 
DI: BAB000000-01717-0200-0004, Rev 02

\section{Mitigation Feature}

Scenario Number: $415010 \quad$ Mitigation Number: 3

Scenario: $\quad$ Accident due to load shifting or equipment/materials protruding from a subsurface transport vehicle (e.g., mantrip, flat car, utility car).

Mitigation Feature:

Restrain equipment and material (e.g., drill steel, loading poles, etc.) so that it cannot change position and protrude outside the transportation window.

Documentation: Training manuals; safety manuals; JSAs.

Remarks:

Mitigation type: $\bigcirc$ Design Feature

Safety Device

Warning Device

Procedure

Training

\section{Mitigation Tracking}

Mitigation

Implementation

Status:

Comments:
Not Implemented

Implemented

Implemented (per T. Rotert, aug98_Materials are secured on flatcars) wp.
Appendix A

Page UI5010.3 


\section{DI: BAB000000-01717-0200-0004, Rev 02}

\section{Mitigation Feature}

Scenario Number: U15010 Mitigation Number: 4

Scenario:

Accident due to load shifting or equipment/materials protruding from a subsurface transport vehicle (e.g., mantrip, flat car, utility car).

Mitigation Feature:

Select equipment/materials/supplies that do not exceed the carrying capacity of cars or the transportation envelope dimensions.

Documentation: 29 CFR 1926.800; training manuals; safety manuals; JSAs.

Remarks:

\begin{tabular}{l|l} 
Mitigation type: & O Design Feature \\
$\bigcirc$ Safety Device \\
$\bigcirc$ Warning Device \\
$\bigcirc$ Procedure \\
Training
\end{tabular}

\section{Mitigation Tracking}

Mitigation Implementation

Status:

Comments:

\section{Not Implemented}

Implemented

Implemented (per T. Rotert, aug98) wp.

Appendix A

Page UI5010.4 
DI: BAB000000-01717-0200-0004, Rev 02

\section{Mitigation Feature}

Scenario Number:

U15010

Mitigation Number:

5

Scenario:

Accident due to load shifting or equipment/materials protruding from a subsurface transport vehicle (e.g., mantrip, flat car, utility car).

Mitigation Feature:

Design adequate clearance for the transportation envelope.

Documentation:

Remarks:

Rev.01, Mit\# 8

Mitigation type:

Design Feature

Safety Device

Warning Device

Procedure

Training

\section{Mítigation Tracking}

\section{Mitigation Implementation Status:}

Comments:
Not Implemented

implemented

Implemented (per T. Rotert, aug98_Track has been previously changed to accommodate envelope) wp.

\section{Appendix A}

Page UI5010.5 


\section{DI: BAB000000-01717-0200-0004, Rev 02}

\section{Mitigation Feature}

Scenario Number:

UI5010

Mitigation Number:

6

Scenario:

Accident due to load shifting or equipment/materials protruding from a subsurface transport vehicle (e.g., mantrip, flat car, utility car).

Mitigation Feature: Explosives will only be transported in "day boxes" which are mounted securely to the vehicle.

Documentation: 29 CFR 1926.800; training manuals; safety manuals; JSAs.

Remarks:

Mitigation type:

Design Feature

Safety Device

Warning Device

Procedure

Training

\section{Mitigation Tracking}

Mitigation Implementation Status:

Comments:
Not Implemented

Implemented

\section{Appendix A}

Page UI5010.6 


\section{DI: BAB000000-01717-0200-0004; Rev 02}

\section{Mitigation Feature}

$\begin{array}{ll}\text { Scenario Number: } & \text { Ul5010 } \\ \text { Scenario: } & \begin{array}{l}\text { Accident due to load shifting or equipment/materials protruding from a subsurface transport } \\ \text { vehicle (e.g., mantrip, flat car, utility car). }\end{array} \\ \text { Mitigation Feature: } & \begin{array}{l}\text { JSA will address proper loading and securing of materials, equipment and supplies on } \\ \text { transport vehicles. }\end{array} \\ \text { Documentation: } & 29 \text { CFR 1926.800; training manuals; safety manuals; JSAs. } \\ \text { Remarks: } & \text { Rev.01, Mit\# } 9 \\ \text { Mitigation type: } & \text { Design Feature } \\ \text { Safety Device } \\ \text { W Warning Device } \\ \text { Procedure } \\ \text { Training }\end{array}$

\section{Mitigation Tracking}

Mitigation Implementation Status:

Comments:
Not Implemented

Implemented

\section{Appendix A}

Page UI5010.7 


\section{Approved}

Scenario Number:

Revision Number:

Revision Date:

Scenario:

Cause, Failure, or Hazardous Event:

Risk Assignment Before Considering Mitigations:

\begin{tabular}{|l|}
\hline$U 15011$ \\
\hline 02 \\
\hline $12 / 17 / 98$ \\
\hline
\end{tabular}

Subsurface vehicle (e.g., front-end loader) accident.

Component failure (e.g., brakes, steering); operator error; pedestrian error; improper storage of equipment or materials.

\begin{tabular}{|lll|}
\hline Frequency Rating: & Consequence Rating: & *Risk Before: \\
A - Frequent & I - Catastrophic & \\
B - Probable & II - Critical & High \\
C - Occasional & III - Marginal & \\
D - Remote & IV - Negligible & \\
E - Improbable & & \\
\hline
\end{tabular}

Risk Designation

After

Applying

Mitigations:

\begin{tabular}{|lll|}
\hline Frequency Rating: & Consequence Rating: & Risk After: \\
A - Frequent & I- Catastrophic & \\
B - Probable & II-Critical & High \\
C - Occasional & OII- Marginal & \\
D-Remote & OIV - Negligible & \\
E- Improbable & & \\
\hline
\end{tabular}

Related Scenarios:

U10043

Prevention

Mitigation

Documentation:
Vehicle specifications; OSHA - 29 CFR 1926 Subparts O \& S; training manuals; operators manuals; maintenance manuals; safety manuals; inspection and maintenance records

\section{Appendix A Page UI5011}




\section{DI: BAB000000-01717-0200-0004; Rev 02}

\section{Mitigation Feature}

Scenario Number:

U15011 Mitigation Number:

Scenario:

Subsurface vehicle (e.g., front-end loader) accident.

Mitigation Feature: Establish maximum safe speed limits for all vehicles.

Documentation: Vehicle specifications; OSHA - 29 CFR 1926 Subparts O \& S; training manuals; operators manuals; maintenance manuals; safety manuals; inspection and maintenance records

Remarks:

Mitigation type:
Design Feature
Safety Device
Warning Device
Procedure
Training

\section{Mitigation Tracking}

Mitigation

Implementation

Status:

Comments:
Not Implemented

implemented

Implemented (per T.Rotert, aug98_Speed limit of $15 \mathrm{mph}$ for locomotive traffic set by CMO and constructor.) wp

\section{Appendix A}

Page UI5011.1 


\section{DI: BAB000000-01717-0200-0004; Rev 02}

\section{Mitigation Feature}

Scenario Number:

U15011

Mitigation Number:

Scenario:

Subsurface vehicle (e.g., front-end loader) accident.

Mitigation Feature: Establish maintenance and inspection schedule for all vehicles.

Documentation:

Vehicle specifications; OSHA - 29 CFR 1926 Subparts O \& S; training manuals; operators manuals; maintenance manuals; safety manuals; inspection and maintenance records

Remarks:

Mitigation type:
Design Feature
Safety Device
Warning Device
Procedure
Training

\section{Mitigation Tracking}

Mitigation Implementation Status:

Comments:
Not Implemented

Implemented

Implemented (per T.Rotert, aug98_ All equipment is inspected during pre-shift inspection prior to operating and undergo periodic preventive maintenance.) wp

\section{Appendix A}

Page UI5011.2 


\section{DI: BAB000000-01717-0200-0004, Rev 02}

\section{Mitigation Feature}

Scenario Number: Ui5011 Mitigation Number: 3

\section{Scenario: $\quad$ Subsurface vehicle (e.g., front-end loader) accident.}

Mitigation Feature: Install onboard fire suppression systems on locomotives and front-end loaders.

Documentation: Vehicle specifications; OSHA - 29 CFR 1926 Subparts O \& S; training manuals; operators manuals; maintenance manuals; safety manuals; inspection and maintenance records

Remarks:

Mitigation type:
Design Feature
Safety Device
Warning Device
Procedure
Training

\section{Mitigation Tracking}

Mitigation Implementation

Status:

Comments:
Not Implemented

Implemented

Implemented (per T. Rotert, aug98 Auto fire suppression installed on locomotives and front end loaders) wp.

\section{Appendix A \\ Page UI5011.3}




\section{DI: BAB000000-01717-0200-0004, Rev 02}

\section{Mitigation Feature}

$\begin{array}{ll}\text { Scenario Number: } & \text { UI5011 Mitigation Number: } 4 \\ \text { Scenario: } & \text { Subsurface vehicle (e.g., front-end loader) accident. }\end{array}$

Mitigation Feature: Provide safety training for vehicle operators and personnel working around vehicles.

Documentation: Vehicle specifications; OSHA - 29 CFR 1926 Subparts O \& S; training manuals; operators manuals; maintenance manuals; safety manuals; inspection and maintenance records

Remarks:

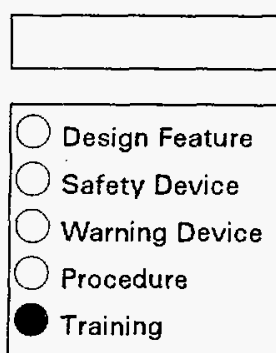

Mitigation type:

\section{Mitigation Tracking}

Mitigation

Implementation

Status:

Not Implemented

Implemented

Comments:

Implemented (per T. Rotert, aug98_Covered in GUT training) wp.

\section{Appendix A}

Page UI5011.4 


\section{DI: BAB000000-01717-0200-0004, Rev 02}

\section{Mitigation Feature}

Scenario Number:

UI5011

Mitigation Number:

5

Scenario:

Subsurface vehicle (e.g., frant-end loader) accident.

Mitigation Feature: Provide headlights on vehicles.

Documentation: Vehicle specifications; OSHA - 29 CFR 1926 Subparts O \& S; training manuals; operators manuals; maintenance manuals; safety manuals; inspection and maintenance records

Remarks:

Mitigation type:

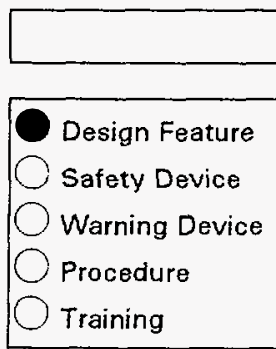

\section{Mitigation Tracking}

Mitigation

Implementation

Status:

Comments:
Not Implemented

Implemented

Implemented \{per T. Rotert, aug98\} wp.

\section{Appendix A \\ Page UI5011.5}




\section{DI: BAB000000-01717-0200-0004; Rev 02}

\section{Mitigation Feature}

Scenario Number: 415011 Mitigation Number: 6

Scenario:

Subsurface vehicle (e.g., front-end loader) accident.

Mitigation Feature: Equip vehicles with automatic "back-up" alarms as appropriate.

Documentation: Vehicle specifications; OSHA - 29 CFR 1926 Subparts O \& S; training manuals; operators manuals; maintenance manuals; safety manuals; inspection and maintenance records

Remarks:

Mitigation type:

$$
\begin{aligned}
& \text { Design Feature } \\
& \text { Safety Device } \\
& \text { Warning Device } \\
& \text { Procedure } \\
& \text { Training }
\end{aligned}
$$

\section{Mitigation Tracking}

Mitigation Implementation Status:

Comments:
Not Implemented

Implemented

Appendix A

Page UI5011.6 


\section{DI: BAB000000-01717-0200-0004, Rev 02}

\section{Mitigation Feature}

Scenario Number: $\quad$ UI5011 Mitigation Number: 7

Scenario:

Subsurface vehicle (e.g., front-end loaderl accident.

Mitigation Feature: Require personnel to stop work and clear the vehicle travel envelop to permit vehicle passage.

Documentation: Vehicle specifications; OSHA - 29 CFR 1926 Subparts O \& S; training manuals; operators manuals; maintenance manuals; safety manuals; inspection and maintenance records

Remarks:

Mitigation type:
Design Feature
Safety Device
Warning Device
Procedure
Training

Mitigation Tracking

Mitigation Implementation Status:

Implemented

Comments: $\quad$ Implemented (per Bob Law, 5May99) jr. 
DI: BAB000000-01717-0200-0004, Rev 02

\section{Mitigation Feature}

Scenario Number: $415011 \quad$ Mitigation Number: 8

Scenario: $\quad$ Subsurface vehicle (e.g., front-end loader) accident.

Mitigation Feature: Storage areas and containers will be located as far outside the travel envelope as possible to reduce the likelihood of damage/explosion/fire in the event of an accident.

Documentation: Vehicle specifications; OSHA - 29 CFR 1926 Subparts O \& S; training manuals; operators manuals; maintenance manuals; safety manuals; inspection and maintenance records

Remarks:

Mitigation type:
Design Feature
Safety Device
Warning Device
Procedure
Training

\section{Mitigation Tracking}

\section{Mitigation Implementation Status:}

Comments:
Not Implemented
Implemented

\section{Appendix A \\ Page UI5011.8}




\section{Scenario Description and Analysis}

\section{Approved}

Scenario Number:

Revision Number:

Revision Date:

Scenario:

Cause, Failure, or Hazardous Event:

Risk Assignment Before Considering Mitigations:

\begin{tabular}{|l|}
\hline 015017 \\
\hline 02 \\
\hline $12 / 17 / 98$ \\
\hline
\end{tabular}
also U(5060 and UI5042).

Ventilation system failure or out-of-tolerance condition results in personnel health hazard (see

Fan failures; exhaust duct system breaks, blockage or rupture; electrical power failure; failure to properly secure equipment or supplies for travel or they exceed travel envelope.

\begin{tabular}{|lll|}
\hline Frequency Rating: & Consequence Rating: & ${ }^{*}$ Risk Before: \\
A - Frequent & $O_{\text {I-Catastrophic }}$ & \\
B - Probable & II - Critical & Medium \\
C - Occasional & III-Marginal & \\
D - Remote & OIV - Negligible & \\
E - Improbable & & \\
\hline
\end{tabular}

Risk Designation

After

Applying

Mitigations:

Related Scenarios:

Prevention

Mitigation

Documentation:

\begin{tabular}{|lll}
\hline Frequency Rating: & Consequence Rating: & Risk After: \\
A - Frequent & $\bigcirc I-$ Catastrophic & \\
B - Probabie & $\bigcirc I I-$ Critical & Extremely Low \\
C - Occasional & III-Marginal & \\
D - Remote & OIV - Negligible & \\
E - Improbable & &
\end{tabular}

U10013, U10106, U15060, U15042

ESF Subsurface Vaneaxial Ventilation Fans Procurement Specification; ESF Ventilation Duct and Fittings Procurement Specification; ventilation fan service manual; operational procedures; ISAs; evacuation procedures; safety manuals; inspection and maintenance records; training manuals \& records.

\section{Appendix A \\ Page UI5017}




\section{DI: BAB000000-01717-0200-0004, Rev 02}

\section{Mitigation Feature}

Scenario Number: $\quad$ U15017 Mitigation Number: 1

Scenario: $\quad$ Ventilation system failure or out-of-tolerance condition results in personnel health hazard (see also U15060 and UI50421.

Mitigation Feature: Ventilation fan procurement specifications shall meet mining industry standards with proven performance in the mining operations.

Documentation: ESF Subsurface Vaneaxial Ventilation Fans Procurement Specification; ESF Ventilation Duct and Fittings Procurement Specification.

Remarks:

Mitigation type:

Design Feature

Safety Device

Warning Device

Procedure

Training

\section{Mitigation Tracking}

Mitigation

Implementation

Status:

Comments:

\section{Not Implemented}

Implemented

Implemented (per T. Rotert, aug98_Fans purchased with required specs) wp.

\section{Appendix A}

Page U15017.1 


\section{DI: BAB000000-01717-0200-0004; Rev 02}

\section{Mitigation Feature}

$\begin{array}{ll}\text { Scenario Number: } & \text { UI5017 } \\ \text { Scenario: } & \begin{array}{l}\text { Ventilation system failure or out-of-tolerance condition results in personnel health hazard (see } \\ \text { also UI5060 and U } 15042) .\end{array} \\ \text { Mitigation Feature: } & \begin{array}{l}\text { Provide ventilation system capacity to maintain adequate air circulation in the event of several } \\ \text { fan failures. }\end{array} \\ \text { Documentation: } & \begin{array}{l}\text { ESF Subsurface Vaneaxial Ventilation Fans Procurement Specification; ESF Ventilation Duct } \\ \text { and Fittings Procurement Specification. }\end{array} \\ \text { Remarks: } & \begin{array}{l}\text { Rev.o1, Mit\# 15 } \\ \text { Mitigation type: }\end{array} \\ \text { Design Feature } \\ \text { Safety Device } \\ \text { Warning Device } \\ \text { Procedure } \\ \text { Training }\end{array}$

\section{Mitigation Tracking}

Mitigation Implementation Status:

Comments:
Not Implemented

Implemented 
DI: BAB000000-01717-0200-0004; Rev 02

\section{Mitigation Feature}

\begin{tabular}{|c|c|c|}
\hline Scenario Number: & Mitigation Number: & 3 \\
\hline Scenario: & $\begin{array}{l}\text { Ventilation system failure or out-of-tolerance condition res } \\
\text { also Ui5060 and U } 15042) \text {. }\end{array}$ & ults in personnel health hazard isee \\
\hline Mitigation Feature: & $\begin{array}{l}\text { Design ventilation system such that individual fans can be } \\
\text { down operations in the tunnel. }\end{array}$ & shut down without having to shut \\
\hline Documentation: & $\begin{array}{l}\text { ESF Subsurface Vaneaxial Ventilation Fans Procurement } S \\
\text { and Fittings Procurement Specification; System Design/Fa }\end{array}$ & $\begin{array}{l}\text { ipecification; ESF Ventilation Duct } \\
\text { in drawings. }\end{array}$ \\
\hline Remarks: & Rev.01, Mit\# 14 & \\
\hline Mitigation type: & $\begin{array}{l}\text { Design Feature } \\
\text { Safety Device } \\
\text { Warning Device } \\
\text { Procedure } \\
\text { Training }\end{array}$ & \\
\hline
\end{tabular}

\section{Mitigation Tracking}

Mitigation Implementation

Status:

Comments:
Not Implemented

Implemented

\section{Appendix A}

Page UI5017.3 


\section{DI: BAB000000-01717-0200-0004; Rev 02}

\section{Mitigation Feature}

Scenario Number: $\quad$ U15017 Mitigation Number: 4

Scenario:

Ventilation system failure or out-of-tolerance condition results in personnel health hazard (see also UI5060 and UI5042).

Mitigation Feature: Perform scheduled preventive maintenance in accordance with manufacturer's recommendations and maintain records.

Documentation: Ventilation fan service manual; operational procedures; JSAs.

Remarks:

Mitigation type:
Design Feature
Safety Device
Warning Device
Procedure
Training

\section{Mitigation Tracking}

Mitigation Implementation

Status:

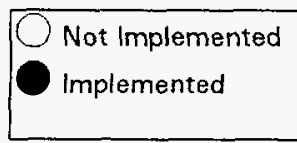

Comments:

\section{Appendix A \\ Page UI5017.4}




\section{DI: BAB000000-01717-0200-0004, Rev 02}

\section{Mitigation Feature}

Scenario Number:

UI5017

Mitigation Number:

5

Scenario:

Ventilation system failure or out-of-tolerance condition results in personnel health hazard (see also UI5060 and Ui5042).

Mitigation Feature: Monitor ventilation system for out-of-tolerance or out-of-specified range conditions. When out of tolerance/range, perform immediate system/equipment repairs or adjustments. Immediate use of respirators by all personnel may be required. Cease work and evacuate.

Documentation:

Ventilation fan service manual; operational procedures; JSAs; evacuation procedures.

Remarks:

Mitigation type:
Design Feature
Safety Device
Warning Device
Procedure
Training

Mitigation Tracking

Mitigation

Implementation

Status:

Comments:
Not Implemented

Implemented

Implemented (per T. Rotert, aug98 Monitoring system is in place) wp. 


\section{DI: BAB000000-01717-0200-0004, Rev 02}

\section{Mitigation Feature}

Scenario Number:

U15017

Mitigation Number:

6

Scenario:

Ventilation system failure or out-of-tolerance condition results in personnel health hazard (see also U15060 and UI5042).

Mitigation Feature: Provide personnel safety training regarding evacuation procedures and respirator use. Execute emergency evacuation plan as necessary.

Documentation: Evacuation procedures; safety training manuals.

Remarks: $\quad$ Rev.01, Mit\# 16

Mitigation type: $\bigcirc$ Design Feature

Safety Device

Warning Device

Procedure

Training

\section{Mitigation Tracking}

Mitigation

Implementation

Status:

Implemented

Comments:

Implemented (per Bob Law, 5 May 99) jr

\section{Appendix A}

Page UI5017.6 
DI: BAB000000-01717-0200-0004; Rev 02

\section{Mitigation Feature}

Scenario Number: $415017 \quad$ Mitigation Number: 7

Scenario: $\quad$ Ventilation system failure or out-of-tolerance condition results in personnel health hazard (see also U15060 and U15042).

Mitigation Feature: Provide reinforcement along ducting ribs and joints to minimize leaks and damage if struck by foreign objects.

Documentation: ESF Ventilation Duct and Fittings Procurement Specification; operational procedures; JSAs.

Remarks:

Mitigation type: $\quad$\begin{tabular}{|l}
$\bigcirc$ Design Feature \\
Safety Device \\
$\bigcirc$ Warning Device \\
$\bigcirc$ Procedure \\
$\bigcirc$ Training
\end{tabular}

\section{Mitigation Tracking}

Mitigation

Implementation

Status:

Comments:
Not Implemented

Implemented

\section{Appendix A}

Page UI5017.7 


\section{DI: BAB000000-01717-0200-0004, Rev 02}

\section{Mitigation Feature}

Scenario Number:

U15017

Mitigation Number:

8

Scenario:

Ventilation system failure or out-of-tolerance condition results in personnel health hazard (see aiso UI5060 and U150421.

Mitigation Feature: Provide enhanced joint seal (i.e., rubber band plus metal duct band) on ventilation ducts.

Documentation:

Remarks:

Mitigation type:
Design Feature
Safety Device
Warning Device
Procedure
Training

\section{Mitigation Tracking}

Mitigation

Implementation

Status:

Not implemented

Implemented

Comments:

Implemented (per T. Rotert, aug98_Neoprene band placed on East-West drift duct.) wp.

\section{Appendix A}

Page UI5017.8 


\section{DI: BAB000000-01717-0200-0004; Rev 02}

\section{Mitigation Feature}

Scenario Number: $\quad$ Ul5017 Mitigation Number: 9

Scenario:

Ventilation system failure or out-of-tolerance condition results in personnel health hazard (see also U15060 and Ui5042).

Mitigation Feature:

Prevent movement of equipment or supplies that exceed travel envelope. JSA will address preparation of equipment or supplies for travel.

Documentation: ESF Subsurface Vaneaxial Ventilation Fans Procurement Specification; ESF Ventilation Duct and Fittings Procurement Specification; ventilation fan service manual; operational procedures;

JSAs; evacuation procedures; safety manuals; inspection and maintenance

Remarks:

Mitigation type:
Design Feature
Safety Device
Warning Device
Procedure
Training

\section{Mitigation Tracking}

Mitigation Implementation Status:

Comments:
Not Implemented

Implemented

Implemented (per T. Rotert, aug98 Maintenance procedures for locomotive.) wp.

\section{Appendix A \\ Page UI5017.9}




\section{DI: BAB000000-01717-0200-0004, Rev 02}

\begin{tabular}{|c|c|}
\hline \multicolumn{2}{|r|}{ Mitigation Feature } \\
\hline Scenario Number: & Mitigation Number: \\
\hline Scenario: & $\begin{array}{l}\text { Ventilation system failure or out-of-tolerance condition results in personnel health hazard (see } \\
\text { also U } 15060 \text { and U15042). }\end{array}$ \\
\hline Mitigation Feature: & Design exhaust ducts to easily repair or replace a section/component. \\
\hline Documentation: & $\begin{array}{l}\text { ESF Subsurface Vaneaxial Ventilation Fans Procurement Specification; ESF Ventilation Duct } \\
\text { and Fittings Procurement Specification. }\end{array}$ \\
\hline Remarks: & \\
\hline Mitigation type: & $\begin{array}{l}\text { Design Feature } \\
\text { Safety Device } \\
\text { Warning Device } \\
\text { Procedure } \\
\text { Training }\end{array}$ \\
\hline
\end{tabular}

\section{Mitigation Tracking}

\section{Mitigation Implementation Status:}

Comments:
Not Implemented

Implemented

Implemented (per T.Rotert, aug98.) wp 
DI: BABO00000-01717-0200-0004, Rev 02

\begin{tabular}{ll}
\hline & \multicolumn{1}{c}{ Mitigation Feature } \\
Scenario Number: & U15017 \\
Scenario: & $\begin{array}{l}\text { Ventilation system failure or out-of-tolerance condition results in personnel health hazard (see } \\
\text { also Ul5060 and U15042). }\end{array}$ \\
Mitigation Feature: & $\begin{array}{l}\text { Install airflow monitors to detect ventilation failure and periodically inspect monitors to ensure } \\
\text { proper functioning. }\end{array}$ \\
Documentation: & Inspection procedures. \\
Remarks: & \\
Mitigation type: & $\begin{array}{l}\text { O Design Feature } \\
\text { safety Device } \\
\text { Warning Device } \\
\text { Procedure } \\
\text { Training }\end{array}$ \\
\hline
\end{tabular}

\section{Mitigation Tracking}

Mitigation Implementation Status:

Comments:
Not Implemented
Implemented

Implemented (per T.Rotert, aug98 Monitors installed in main tunnel and on the TBM.) wp 


\section{DI: BAB000000-01717-0200-0004, Rev 02}

\section{Mitigation Feature}

Scenario Number:

UI5017 Mitigation Number:

12

Scenario:

Ventilation system failure or out-of-tolerance condition results in personnel health hazard isee also UI5060 and UI5042).

Mitigation Feature: Perform periodic inspection of the exhaust ducting system.

Documentation: Inspection and maintenance procedures.

Remarks:

Mitigation type:
Design Feature
Safety Device
Warning Device
Procedure
Training

\section{Mitigation Tracking}

\section{Mitigation Implementation Status:}

Comments:
Not Implemented

Impiemented

Implemented (per T.Rotert, aug98.) wp

\section{Appendix A}

Page UI5017.12 


\section{Scenario Description and Analysis}

\section{Approved}

Scenario Number: Revision Number: Revision Date:

\begin{tabular}{|l|}
\hline$U 15025$ \\
\hline 02 \\
\hline $12 / 17 / 98$ \\
\hline
\end{tabular}

SSA: East-West Drift

Location: East-West Drift

Scenario:

Compressed air line rupture results in personnel injury.

Cause, Failure, or Hazardous Event:

Risk Assignment Before Considering Mitigations:

\section{Pipe rupture (e.g., due to over-pressure or impact on piping); pipe fitting failure}

\begin{tabular}{|lll|}
\hline Frequency Rating: & Consequence Rating: & *Risk Before: \\
A - Frequent & $\bigcirc I$ - Catastrophic & \\
B - Probable & II - Critical & Medium \\
C - Occasional & III - Marginal & \\
D-Remote & IV - Negligible & \\
E - Improbable & & \\
\hline
\end{tabular}

Risk Designation

After

Applying

Mitigations:

\begin{tabular}{|lll|}
\hline Frequency Rating: & Consequence Rating: & Risk After: \\
O - Frequent & $\bigcirc I-$ Catastrophic & \\
B - Probable & $\bigcirc I I-$ Critical & Extremely Low \\
C - Occasional & III- Marginal & \\
D - Remote & IV - Negligible & \\
E - Improbable & & \\
\hline
\end{tabular}

Related Scenarios:

U10020

Prevention

System specifications; inspection/maintenance/test records; Design drawing:

Mitigation

BABFAG000-01717-2100-45302, Subsurface Compressed Air System Flow Diagram.

Documentation:

\section{Appendix A \\ Page UI5025}




\section{DI: BAB000000-01717-0200-0004, Rev 02}

\section{Mitigation Feature}

Scenario Number:

015025

Mitigation Number:

1

Scenario:

Compressed air line rupture results in personnel injury.

Mitigation Feature:

Design and install air lines and pressure relief valves to applicable codes \& standards.

Documentation:

System specifications; Design drawing: BABFAG000-01717-2100-45302.

Remarks:

Mitigation type:

$$
\begin{aligned}
& \text { Design Feature } \\
& \text { Safety Device } \\
& \text { Warning Device } \\
& \text { Procedure } \\
& \text { Training }
\end{aligned}
$$

\section{Mitigation Tracking}

Mitigation Implementation

Status:

Comments:
Not Implemented

implemented

Implemented (per T. Rotert, aug98) wp.

\section{Appendix A}

Page UI5025.1 


\section{DI: BAB000000-01717-0200-0004; Rev 02}

\section{Mitigation Feature}

Scenario Number: $u 15025 \quad$ Mitigation Number: 2

Scenario: $\quad$ Compressed air line rupture results in personnel injury.

Mitigation Feature: Position piping out of vehicle, equipment and material travel envelope and path.

Documentation: Design drawing: BABFAG000-01717-2100-45302, Subsurface Compressed Air System Flow Diagram.

Remarks:

Mitigation type:
Design Feature
Safety Device
Warning Device
Procedure
Training

Mitigation Tracking

Mitigation

Implementation

Status:

Comments:
Not Implemented

Implemented

Implemented (per T.Rotert, aug98) wp.

Appendix A

Page UI5025.2 
DI: BAB000000-01717-0200-0004; Rev 02

\section{Mitigation Feature}

$\begin{array}{ll}\text { Scenario Number: } & \text { U15025 } \\ \text { Scenario: } & \text { Mitigation Number: } \\ \text { Mitigation Feature: } & \begin{array}{l}\text { Provide safety training for personnel regarding high pressure fitting leak hazards and locations } \\ \text { of shut off valves. }\end{array} \\ \text { Documentation: } & \begin{array}{l}\text { Safety manuals; maintenance manuals; training manuals; Design drawing: } \\ \text { BABFAGooo-01717-2100-45302, Subsurface Compressed Air System Flow Diagram }\end{array} \\ \text { Remarks: } & \\ \text { Mitigation type: } & \text { Design Feature } \\ & \begin{array}{l}\text { Safety Device } \\ \text { Warning Device }\end{array} \\ \text { Procedure } \\ \text { Training }\end{array}$

\section{Mitigation Tracking}

Mitigation Implementation Status:

Not Implemented

Implemented

Comments: 


\section{DI: BAB000000-01717-0200-0004; Rev 02}

\begin{tabular}{|c|c|}
\hline & Mitigation Feature \\
\hline Scenario Number: & Mitigation Number: \\
\hline Scenario: & Compressed air line rupture results in personnel injury. \\
\hline Mitigation Feature: & Establish inspection and maintenance schedules (for valves, pipes, etc.). \\
\hline Documentation: & $\begin{array}{l}\text { Maintenance manuals; training manuals; system specifications; inspection/maintenance/test } \\
\text { records; Design drawing: BABFAGO00-01717-2100-45302, Subsurface Compressed Air } \\
\text { System Flow Diagram. }\end{array}$ \\
\hline Remarks: & Rev.02, Mit\# 5 \\
\hline Mitigation type: & $\begin{array}{l}\text { Design Feature } \\
\text { Safety Device } \\
\text { Warning Device } \\
\text { Procedure } \\
\text { Training }\end{array}$ \\
\hline
\end{tabular}

\section{Mitigation Tracking}

Mitigation

Implementation

Status:

Comments:
Not Implemented

Implemented

Pending Maximo implementation. (per Bob Law, 5 May 99) JLR

Appendix A

Page UI5025.4 
DI: BAB000000-01717-0200-0004, Rev 02

\section{Scenario Description and Analysis}

\section{Approved}

Scenario Number:

Revision Number:

Revision Date:

Scenario:

Cause, Failure, or Hazardous Event:

Risk Assignment Before Considering Mitigations:

Risk Designation

After

Applying

Mitigations:

Related Scenarios:

Prevention

Mitigation

Documentation:

\begin{tabular}{|l|}
\hline U15028 \\
\hline 02 \\
\hline $12 / 17 / 98$ \\
\hline
\end{tabular}

SSA: East-West Drift

Location: East-West Drift

Unsafe vehicles (e.g., improper maintenance); Fuel source (diesel fuel) exposed to ignition source (e.g., spark, flame); Use of unauthorized vehicle underground; Poor housekeeping; Lack of storage space/facilities; Failure to adhere to safety procedures.

\begin{tabular}{|lll|}
\hline Frequency Rating: & Consequence Rating: & *Risk Before: \\
A - Frequent & $\bigcirc I$ - Catastrophic & \\
B - Probable & $\bigcirc I I$ - Critical & Medium \\
C - Occasional & $\bigcirc I I I-$ Marginal & \\
D-Remote & $\bigcirc I V$ - Negligible & \\
E-Improbable & & \\
\hline
\end{tabular}

\begin{tabular}{|lll|}
\hline Frequency Rating: & Consequence Rating: & Risk After: \\
A - Frequent & I - Catastrophic & \\
B - Probable & $O I I-$ Critical & Extremely LoW \\
C - Occasional & III- Marginal & \\
D - Remote & IV - Negligible & \\
E- Improbable & & \\
\hline
\end{tabular}

U10055

OSHA 29 CFR 1926.800; NFPA Fire Protection Rules and Regulations; maintenance manuals and safety manual; inspection and maintenance records.

\section{Appendix A \\ Page UI5028}




\section{DI: BABO00000-01717-0200-0004, Rev 02}

\section{Mitigation Feature}

Scenario Number: U15028 Mitigation Number:

Scenario: $\quad$ Fire involving subsurface vehicle (e.g., front-end loader) or temporarily stored materials.

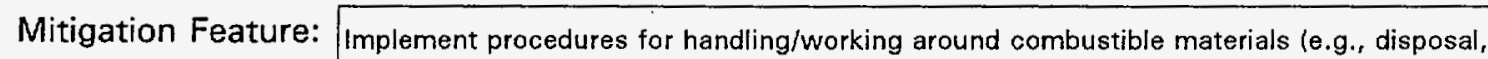
proper storage, no smoking).

Documentation: $\quad$ OSHA 29 CFR 1926.800; NFPA Fire Protection Rules and Regulatians; maintenance manuals and safety manual; inspection and maintenance records

Remarks:

Mitigation type: $\bigcirc$ Design Feature

Safety Device

Warning Device

Procedure

Training

Mitigation Tracking

Mitigation Implementation

Status:

Comments:
Not Implemented

implemented

Implemented lper T. Rotert, aug98_No smoking provisions are in place during combustible operations and handling\} wp. 
DI: BAB000000-01717-0200-0004; Rev 02

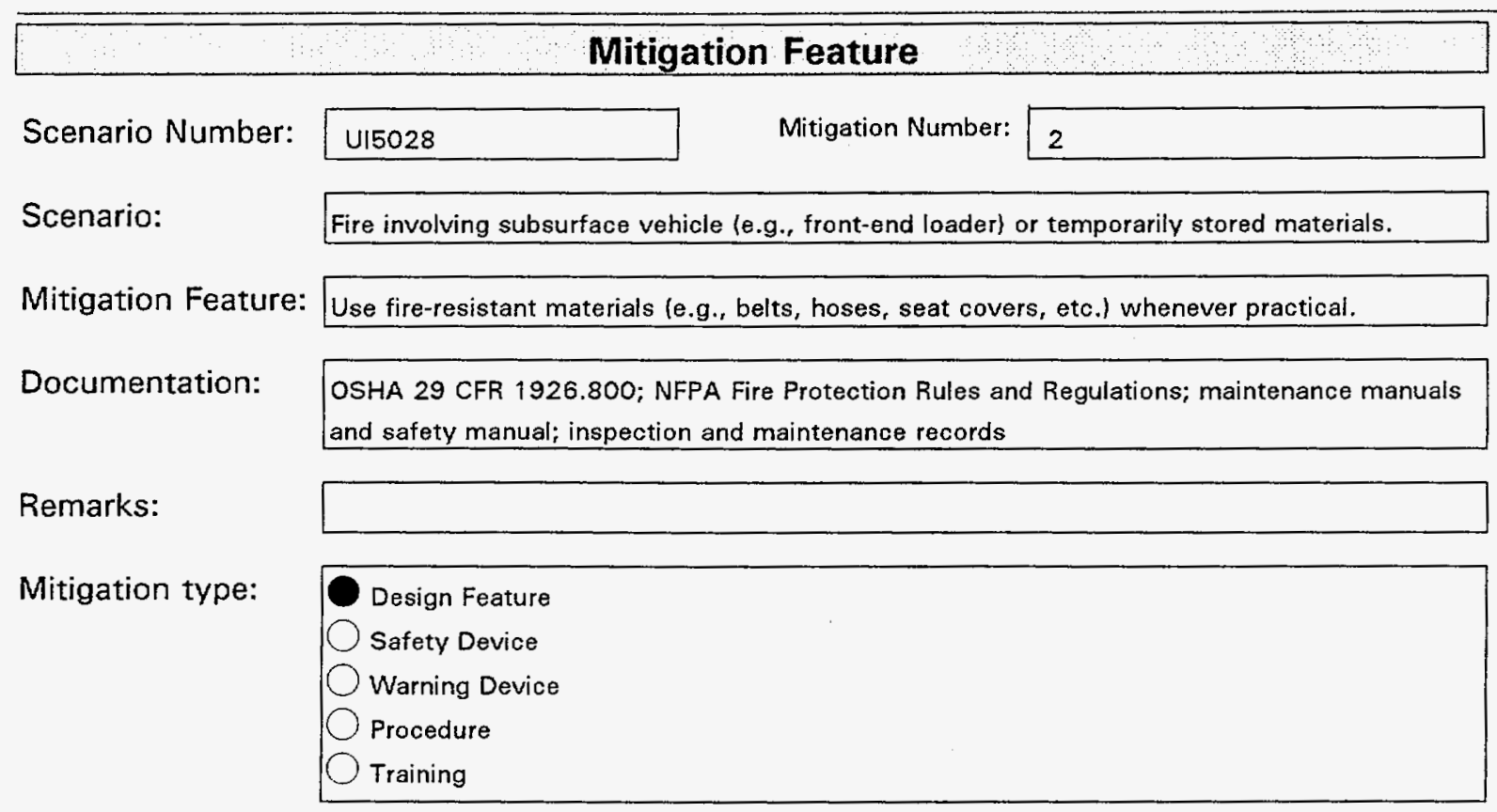

\section{Mitigation Tracking}

Mitigation Implementation

Status:

Not Implemented

Implemented

Comments:

Implemented (per T. Rotert, aug98) wp.

\section{Appendix A}

Page UI5028.2 
DI: BAB000000-01717-0200-0004, Rev 02

\begin{tabular}{|c|c|}
\hline \multicolumn{2}{|r|}{ Mitigation Feature } \\
\hline Scenario Number: & Mitigation Number: \\
\hline Scenario: & Fire involving subsurface vehicle (e.g., front-end loader) or temporarily stored materials. \\
\hline Mitigation Feature: & Provide approved fuel storage containers. \\
\hline Documentation: & $\begin{array}{l}\text { OSHA } 29 \text { CFR 1926.800; NFPA Fire Protection Rules and Regulations; maintenance manuals } \\
\text { and safety manual; inspection and maintenance records }\end{array}$ \\
\hline Remarks: & \\
\hline Mitigation type: & $\begin{array}{l}\text { Design Feature } \\
\text { Safety Device } \\
\text { Warning Device } \\
\text { Procedure } \\
\text { Training }\end{array}$ \\
\hline
\end{tabular}

\section{Mítigation Tracking}

Mitigation Implementation Status:

Comments:
Not Implemented

Implemented 


\section{DI: BAB000000-01717-0200-0004; Rev 02}

\section{Mitigation Feature}

Scenario Number:

U15028

Mitigation Number:

4

Scenario:

Fire involving subsurface vehicle (e.g.r front-end loader) or temporarily stored materials.

Mitigation Feature: Limit diesel fuel storage volume to no more than 24-hour supply.

Documentation:

OSHA 29 CFR 1926.800; NFPA Fire Protection Rules and Regulations; maintenance manuals and safety manual; inspection and maintenance records

Remarks:

Mitigation type:
Design Feature
Safety Device
Warning Device
Procedure
Training

\section{Mitigation Tracking}

Mitigation

Implementation

Status:

Comments:
Not Implemented

Implemented

Implemented (per T. Rotert, aug98) wp.

\section{Appendix A}

Page UI5028.4 
DI: BAB000000-01717-0200-0004, Rev 02

\section{Mitigation Feature}

Scenario Number:

UI5028 Mitigation Number:

5

Scenario:

Fire involving subsurface vehicle (e.g., front-end loader) or temporarily stored materials.

Mitigation Feature:

Provide safety training (e.g., rules and regulations concerning the employee's safety and the safety of others, parking of vehicles).

Documentation: OSHA 29 CFR 1926, JSAs

Remarks:

Mitigation type:
Design Feature
Safety Device
Warning Device
$\bigcirc$ Procedure
Training

Mitigation Tracking

Mitigation Implementation

Status:

Not Implemented

Implemented

Comments:

Implemented (per T. Rotert, aug98) wp.

\section{Appendix A \\ Page UI5028.5}




\section{Dl: BAB000000-01717-0200-0004, Rev 02}

\section{Mitigation Feature}

Scenario Number: U15028 Mitigation Number:

Scenario: $\quad$ Fire involving subsurface vehicle (e.g., front-end loader) or temporarily stored materials.

Mitigation Feature: Install portable extinguishers and hose bibs in the ECRB.

Documentation:

OSHA 29 CFR 1926.800; NFPA Fire Protection Rules and Regulations; maintenance manuals and safety manual; inspection and maintenance records

Remarks:

Mitigation type:
Design Feature
Safety Device
Warning Device
Procedure
Training

\section{Mitigation Tracking}

Mitigation Implementation

Status:

Comments:
Not Implemented

Implemented

Implemented (per T. Rotert, aug98) wp. 
DI: BAB000000-01717-0200-0004, Rev 02

\begin{tabular}{|c|c|}
\hline \multicolumn{2}{|r|}{ Mitigation Feature } \\
\hline Scenario Number: & Mitigation Number: \\
\hline Scenario: & Fire involving subsurface vehicle (e.g., front-end loader) or temporarily stored materials. \\
\hline Mitigation Feature: & $\begin{array}{l}\text { Provide onboard fire suppression systems (e.g., installed or portable fire extinguishers) on } \\
\text { locomotives and front-end loaders. }\end{array}$ \\
\hline Documentation: & $\begin{array}{l}\text { OSHA } 29 \text { CFR 1926.800; NFPA Fire Protection Rules and Regulations; maintenance manuals } \\
\text { and safety manual; inspection and maintenance records }\end{array}$ \\
\hline Remarks: & \\
\hline Mitigation type: & $\begin{array}{l}\text { Design Feature } \\
\text { Safety Device } \\
\text { warning Device } \\
\text { Procedure } \\
\text { Training }\end{array}$ \\
\hline
\end{tabular}

\section{Mitigation Tracking}

Mitigation Implementation

Status:

Not Implemented

Implemented

Comments:

Implemented (per T. Rotert, aug98) wp.

Appendix A

Page U15028.7 
DI: BAB000000-01717-0200-0004, Rev 02

\section{Mitigation Feature}

Scenario Number: U15028 Mitigation Number: 8

Scenario: $\quad$ Fire involving subsurface vehicle (e.g., front-end loader) or temporarily stored materials.

Mitigation Feature: Establish vehicle inspection and maintenance schedules.

Documentation: $\quad$ OSHA 29 CFR 1926.800; NFPA Fire Protection Rules and Regulations; maintenance manuals and safety manual; inspection and maintenance records

Remarks:

Mitigation type:
Design Feature
Safety Device
Warning Device
Procedure
Training

\section{Mitigation Tracking}

Mitigation

Implementation

Status:

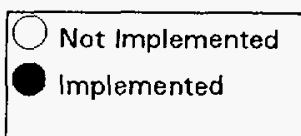

Comments:

Implemented (per T. Rotert, aug98) wp.

\section{Appendix A}

Page UI5028.8 
DI: BAB000000-01717-0200-0004, Rev 02

\section{Mitigation Feature}

Scenario Number:

$\mathrm{U} 15028$

Mitigation Number

9

Scenario:

Fire involving subsurface vehicle (e.g., front-end loader) or temporarily stored materials.

Mitigation Feature: Evacuate in accordance with Emergency Evacuation Plan. Use self rescuer as required.

Documentation:

OSHA 29 CFR 1926.800; NFPA Fire Protection Rules and Regulations; maintenance manuals and safety manual; inspection and maintenance records

Remarks:

Mitigation type:
Design Feature
Safety Device
Warning Device
Procedure
Training

\section{Mitigation Tracking}

Mitigation

Implementation

Status:

Comments:
Not Implemented

implemented

Appendix A

Page U15028.9 


\section{DI: BAB000000-01717-0200-0004, Rev 02}

\section{Mitigation Feature}

$\begin{array}{ll}\text { Scenario Number: } & \text { Ul5028 } \\ \text { Scenario: } & \text { Mire involving subsurface vehicle (e.g., front-end loader) or temporarily stored materials. } \\ \text { Mitigation Feature: } & \begin{array}{l}\text { Ventilation system exhausts smoke and confines dispersion. Also provides fresh air source up } \\ \text { wind of a fire. }\end{array} \\ \text { Documentation: } & \begin{array}{l}\text { OSHA } 29 \text { CFR 1926.800; NFPA Fire Protection Rules and Regulations; maintenance manuals } \\ \text { and safety manual; inspection and maintenance records }\end{array} \\ \text { Remarks: } & \\ \text { Mitigation type: } & \text { Design Feature } \\ \text { Safety Device } \\ \text { Warning Device } \\ \text { Procedure } \\ \text { Training }\end{array}$

\section{Mitigation Tracking}

Mitigation

Implementation

Status:

Comments:
Not Implemented

Implemented

Appendix A

Page UI5028.10 


\section{Scenario Description and Analysis}

\section{Approved}

Scenario Number:

Revision Number:

Revision Date:

Scenario:

Cause, Failure, or Hazardous Event:

Risk Assignment Before Considering Mitigations:

\begin{tabular}{l} 
Risk Designation \\
After \\
\hline Applying \\
Mitigations:
\end{tabular}

Related Scenarios:

Prevention Mitigation Documentation:

\begin{tabular}{|l|}
\hline U15030 \\
\hline 02 \\
\hline $12 / 17 / 98$ \\
\hline
\end{tabular}

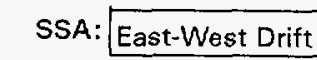

Location: East-West Drift

Fire due to failure/short/arc/overload of electrical switchgear or other electrical equipment (e.g., transformer, electrical panel/box, electrical wire or cable).

Bad winding insulation; short circuit inside electrical panel/box; insulation failure (e.g., cracked frayed); cable breach; electrical overload, impact damage to electrical devices.

\begin{tabular}{|lll|}
\hline Frequency Rating: & Consequence Rating: & *Risk Before: \\
A - Frequent & I-Catastrophic & \\
B - Probable & II-Critical & Medium \\
C - Occasional & $O I I$ - Marginal & \\
D - Remote & $O I V$ - Negligible & \\
E - Improbable & & \\
\hline
\end{tabular}

\begin{tabular}{|lll|}
\hline Frequency Rating: & Consequence Rating: & Risk After: \\
A - Frequent & $O_{1-\text { Catastrophic }}$ & \\
B - Probable & $O_{I I-\text { Critical }}$ & \\
C - Occasional & Extremely Low \\
D- Remote & IIV- Marginal & \\
E - Improbable & & \\
\hline
\end{tabular}

U10037, U10040

ANSI/IEEE C2-93, OSHA 29 CFR 1926; National Electrical Safety Code; National Electrical Code; NEMA and UL standards; system specifications; maintenance manuals; safety manuals; inspection and maintenance records 


\section{DI: BAB000000-01717-0200-0004, Rev 02}

\section{Mitigation Feature}

Scenario Number: UI5030 Mitigation Number: 1

Scenario: $\quad$ Fire due to failure/short/arc/overioad of electrical switchgear or other electrical equipment (e.g., transformer, electrical panel/box, electrical wire or cable).

Mitigation Feature: Design electrical systems/components to applicable codes $\&$ standards.

Documentation: ANSI/IEEE C2-93, OSHA 29 CFR 1926; National Electrical Safety Code; National Electrical Code; NEMA and UL standards; system specifications; maintenance manuals; safety manuals; inspection and maintenance records

Remarks:

Mitigation type:

Design Feature

Safety Device

Warning Device

Procedure

$\bigcirc$ Training

\section{Mitigation Tracking}

Mitigation

Implementation

Status:

Comments:
Not Implemented

Implemented 


\section{DI: BAB000000-01717-0200-0004, Rev 02}

\section{Mitigation Feature}

Scenario Number: $\quad$ U15030 Mitigation Number: 2

Scenario: $\quad$ Fire due to failure/short/arc/overload of electrical switchgear or other electrical equipment (e.g., transformer, electrical panel/box, electrical wire or cable).

Mitigation Feature: Conduct inspections during electrical equipment installation and connection.

Documentation: ANSI/IEEE C2-93, OSHA 29 CFR 1926; National Electrical Safety Code; National Electrical Code; NEMA and UL standards; system specifications; maintenance manuals; safety manuals; inspection and maintenance records

Remarks:

Mitigation type:
Design Feature
Safety Device
Warning Device
Procedure
Craining

\section{Mitigation Tracking}

Mitigation Implementation Status: Not Implemented

Implemented

Comments:

Implemented (per T.Rotert, aug98.) wp

\section{Appendix A}

Page UI5030.2 
DI: BAB000000-01717-0200-0004; Rev 02

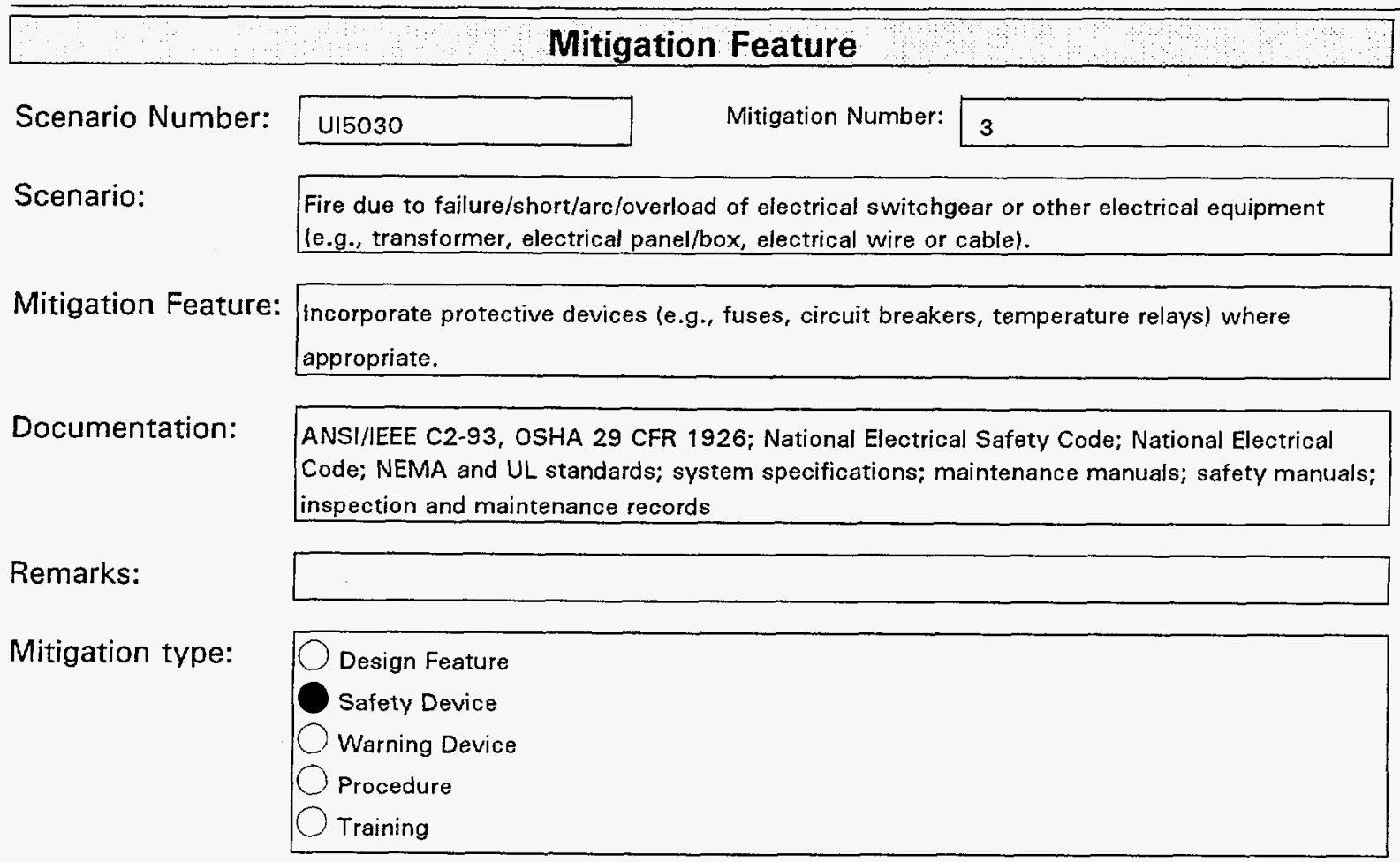

\section{Mitigation Tracking}

\section{Mitigation Implementation Status:}

Comments:
Not Implemented

Implemented 


\section{DI: BAB000000-01717-0200-0004; Rev 02}

\section{Mitigation Feature}

Scenario Number:

U15030 Mitigation Number:

4

Scenario:

Fire due to failure/short/arc/overload of electrical switchgear or other electrical equipment (e.g., transformer, electrical panel/box, electrical wire or cable).

Mitigation Feature: Field test according to applicable specifications.

Documentation: ANSI/IEEE C2-93, OSHA 29 CFR 1926; National Electrical Safety Code; National Electrical Code; NEMA and UL standards; system specifications; maintenance manuals; safety manuals; inspection and maintenance records

Remarks:

Mitigation type:
Design Feature
Safety Device
Warning Device
Procedure
Training

\section{Mitigation Tracking}

Mitigation Implementation Status:

Comments:
Not implemented

Implemented

Appendix A

Page UI5030.4 


\section{DI: BAB000000-01717-0200-0004; Rev 02}

\section{Mitigation Feature}

Scenario Number: $\quad$ UI5030 Mitigation Number: 5

Scenario:

Fire due to failure/short/arc/overload of electrical switchgear or other electrical equipment 〈e.g., transformer, electrical panel/box, electrical wire or cable〉.

Mitigation Feature: Provide portable extinguishers and hose bibs in the ECRB.

Documentation: $\quad$ ANSI/IEEE C2-93, OSHA 29 CFR 1926; National Electrical Safety Code; National Electrical Code; NEMA and UL standards; system specifications; maintenance manuals; safety manuals; inspection and maintenance records

Remarks:

Mitigation type:
Design Feature
Safety Device
Warning Device
Procedure
Training

\section{Mitigation Tracking}

Mitigation Implementation

Status:

Comments:
Not Implemented

Implemented 


\section{DI: BAB000000-01717-0200-0004, Rev 02}

\section{Mitigation Feature}

Scenario Number:

415030

Mitigation Number:

6

Scenario:

Fire due to failure/short/arc/overload of electrical switchgear or other electrical equipment (e.g., transformer, electrical panel/box, electrical wire or cable).

Mitigation Feature: Establish inspection and maintenance schedule.

Documentation:

ANSI/IEEE C2-93, OSHA 29 CFR 1926; National Electrical Safety Code; National Electrical Code; NEMA and UL standards; system specifications; maintenance manuals; safety manuals; inspection and maintenance records

Remarks:

Mitigation type:
Design Feature
Safety Device
Warning Device
Procedure
Training

\section{Mitigation Tracking}

Mitigation Implementation Status:

Comments:

\section{Not Implemented}

O Implemented

Pending Maximo implementation (per Bob Law, 5 May 99) jr

\section{Appendix A \\ Page UI5030.6}


DI: BABO00000-01717-0200-0004, Rev 02

\section{Mitigation Feature}

Scenario Number:

U15030

Mitigation Number:

7

Scenario:

Fire due to failure/short/arc/overload of electrical switchgear or other electrical equipment (e.g., transformer, electrical panel/box, electrical wire or cable).

Mitigation Feature: Ensure cable meets applicable codes and standards.

Documentation:

ANSI/IEEE C2-93, OSHA 29 CFR 1926; National Electrical Safety Code; National Electrical Code; NEMA and UL standards; system specifications; maintenance manuals; safety manuals; inspection and maintenance records

Remarks:

Mitigation type:

$$
\begin{aligned}
& \text { Safety Device } \\
& \text { Warning Device } \\
& \text { Procedure } \\
& \text { Training }
\end{aligned}
$$

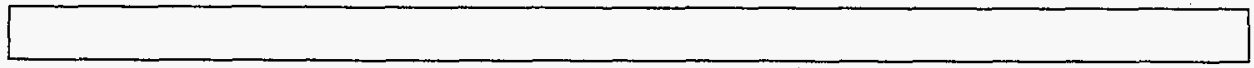

\section{Mitigation Tracking}

Mitigation

Implementation

Status:

Comments:
Not Implemented

Implemented

Implemented (per T. Rotert, aug98) wp.

\section{Appendix A}

Page UI5030.7 


\section{DI: BAB000000-01717-0200-0004, Rev 02}

\section{Mitigation Feature}

Scenario Number: UI5030 Mitigation Number: 8

Scenario:

Fire due to failure/short/arc/overload of electrical switchgear or other electrical equipment (e.g., transformer, electrical panel/box, electrical wire or cable).

Mitigation Feature: Provide fire safety training.

Documentation:

ANSI/IEEE C2-93, OSHA 29 CFR 1926; National Electrical Safety Code; National Electrical Code; NEMA and UL standards; system specifications; maintenance manuals; safety manuals; inspection and maintenance records

Remarks:

Mitigation type:
Design Feature
Safety Device
Warning Device
Procedure
Training

\section{Mitigation Tracking}

Mitigation Implementation Status:

Comments:
Not Implemented

Implemented

Implemented (per T. Rotert, aug98) wp.

\section{Appendix A}

Page UI5030.8 


\section{Scenario Description and Analysis}

\section{Approved}

Scenario Number:

Revision Number:

Revision Date:

Scenario:

Cause, Failure, or Hazardous Event:

\section{Risk Assignment \\ Before Considering Mitigations:}

\begin{tabular}{|l|}
\hline U15042 \\
\hline 02 \\
\hline $12 / 17 / 98$ \\
\hline
\end{tabular}

Air Quality - Toxic fumes (e.g., carbon monoxide, carbon dioxide, nitrogen dioxide, NOx) from diesel exhaust results in a personnel health hazard (see also U15070).

Diesel vehicle exhaust system failure or out-of-tolerance condition; ventilation system failure or out-of-tolerance condition.

\begin{tabular}{|lll|}
\hline Frequency Rating: & Consequence Rating: & *Risk Before: \\
A - Frequent & I - Catastrophic & \\
B - Probable & II - Critical & High \\
C - Occasional & III - Marginal & \\
D - Remote & OIV - Negligible & \\
E - Improbable & & \\
\hline
\end{tabular}

Risk Designation

After

Applying

Mitigations:

Related Scenarios:

Prevention

Mitigation

Documentation:

\begin{tabular}{|lll|}
\hline Frequency Rating: & Consequence Rating: & Risk After: \\
A - Frequent & OI-Catastrophic & \\
B - Probable & $\bigcirc I I-$ Critical & Extremely Low \\
C - Occasional & III - Marginal & \\
D - Remote & IV - Negligible & \\
E - Improbable & & \\
\hline
\end{tabular}

U15070

Vehicle operating and maintenance procedures, manuals and records; OSHA workplace exposure limits; JSA(s); Training records. 


\section{DI: BAB000000-01717-0200-0004, Rev 02}

\section{Mitigation Feature}

Scenario Number:

$\mathrm{U15042}$

Mitigation Number:

1

Scenario:

Air Quality - Toxic fumes (e.g., carbon monoxide, carbon dioxide, nitrogen dioxide, NOx) from diesel exhaust results in a personnel health hazard (see also U15070).

Mitigation Feature: JSAs, procedures and/or training will address hazards associated with operational activities.

Documentation:

Vehicle operating and maintenance procedures, manuals and records; OSHA workplace exposure limits; JSA $(s)$; training records

Remarks:

Mitigation type:
Design Feature
Safety Device
Warning Device
Procedure
Training

\section{Mitigation Tracking}

\section{Mitigation Implementation Status:}

Comments:
Not Implemented

Implemented

Implemented (per T. Rotert, aug98) wp.

\section{Appendix A}

Page UI5042.1 
DI: BAB000000-01717-0200-0004, Rev 02

\section{Mitigation Feature}

Scenario Number:

U15042

Mitigation Number:

2

Scenario:

Air Quality - Toxic fumes (e.g., carbon monoxide, carbon dioxide, nitrogen dioxide, NOx) from diesel exhaust results in a personnel health hazard (see also U15070).

Mitigation Feature:

Ventilation system removes exhaust fumes and confines dispersion. Also provides fresh air source up wind of the diesel equipment.

Documentation:

Vehicle operating and maintenance procedures, manuals and records; OSHA workplace exposure limits; JSA(s); training records

Remarks:

Mitigation type:

\section{Mitigation Tracking}

Mitigation

Implementation

Status:

Comments:
Design Feature

Safety Device

Warning Device

Procedure

Training
Not Implemented

Implemented 
DI: BAB000000-01717-0200-0004; Rev 02

\section{Mitigation Feature}

Scenario Number: $\quad$ UI5042 Mitigation Number: 3

Scenario:

Air Quality - Toxic fumes ie.g., carbon monoxide, carbon dioxide, nitrogen dioxide, NOx) from diesel exhaust results in a personnel health hazard (see also U15070).

Mitigation Feature:

Monitor ventilation system for out-of-tolerance or out-of-specified range conditions. When out of tolerance/range, perform immediate system/equipment repairs or adjustments. Immediate use of respirators by all personnel may be required. Cease work and evacuate if fumes rise to life threatening levels.

Documentation: OSHA workplace exposure limits; JSA(s)

Remarks:

Mitigation type:
Design Feature
Safety Device
Warning Device
Procedure
Training

\section{Mitigation Tracking}

Mitigation Implementation

Status:

Comments:
Not Implemented

Implemented
Implemented (per T. Rotert, aug98 Maintenance procedures for lacomotive.) wp. 


\section{DI: BAB000000-01717-0200-0004, Rev 02}

\section{Mitigation Feature}

Scenario Number: $415042 \quad$ Mitigation Number: 4

Scenario: $\quad$ Air Quality - Toxic fumes (e.g., carbon monoxide, carbon dioxide, nitrogen dioxide, NOx) from diesel exhaust results in a personnel health hazard (see also U15070).

Mitigation Feature: Minimize idling of diesel engines.

Documentation: Vehicle operating and maintenance procedures, manuals and records; OSHA workplace exposure limits; JSA(s); training records

Remarks:

Mitigation type:
Design Feature
Safety Device
Warning Device
Procedure
Training

\section{Mitigation Tracking}

Mitigation Implementation Status:

Not Implemented

Implemented

Comments: 


\section{DI: BAB000000-01717-0200-0004, Rev 02}

\section{Mitigation Feature}

Scenario Number: $\quad$ U15042 Mitigation Number:

5

Scenario:

Air Quality - Toxic fumes (e.g., carbon monoxide, carbon dioxide, nitrogen diaxide, NOx) from diesel exhaust results in a personnel health hazard (see also UI5070).

Mitigation Feature: Install scrubbers (catalytic converters) on subsurface diesel equipment/vehicles.

Documentation: Vehicle operating and maintenance procedures, manuals and records; OSHA workplace exposure limits; JSA(s); training records

Remarks:

Mitigation type:
Design Feature
Safety Device
Warning Device
Procedure
Training

\section{Mitigation Tracking}

Mitigation Implementation

Status:

Comments:

\section{Not implemented}

Implemented 


\section{DI: BAB000000-01717-0200-0004; Rev 02}

\section{Mitigation Feature}

$\begin{array}{ll}\text { Scenario Number: } & \text { Ul5042 } \\ \text { Scenario: } & \begin{array}{l}\text { Air Quality - Toxic fumes (e.g., carbon monoxide, carbon dioxide, nitrogen dioxide, NOx) from } \\ \text { diesel exhaust results in a personnel health hazard (see also Ui5070). }\end{array} \\ \text { Mitigation Feature: } & \text { Establish vehicle inspection \& maintenance schedule, } \\ \text { Documentation: } & \begin{array}{l}\text { Vehicle operating and maintenance procedures, manuals and records; OSHA workplace } \\ \text { exposure limits; JSA(s); training records }\end{array} \\ \text { Remarks: } & \\ \text { Mitigation type: } & \text { Design Feature } \\ \text { Safety Device } \\ \text { Warning Device } \\ \text { Procedure } \\ \text { Training }\end{array}$

\section{Mitigation Tracking}

\section{Mitigation Implementation Status:}

Comments:

Not Implemented
Implemented

Appendix A

Page UI5042.6 


\title{
Scenario Description and Analysis
}

\section{Approved}

Scenario Number:

Revision Number:

Revision Date:

\begin{tabular}{|l|}
\hline U15046 \\
\hline 02 \\
\hline $12 / 17 / 98$ \\
\hline
\end{tabular}

\author{
SSA: East-West Drift \\ Location: Starter Tunnel
}

Scenario:

Train/locomotive derailment at turnout (intersection of East-West Starter Tunnel and TS Main Drift\}.

Cause, Failure, or Hazardous Event:

Rail failure, switch malfunction, operator error, failure to adhere to safety procedures and rules.

Risk Assignment

Before Considering

Mitigations:

\begin{tabular}{|lll|}
\hline Frequency Rating: & Consequence Rating: & *Risk Before: \\
O A - Frequent & $\bigcirc I$ - Catastrophic & \\
B - Probable & $\bigcirc I I-$ Critical & Medium \\
C - Occasional & $\bigcirc I I$ - Marginal & \\
D-Remote & $O I V-$ Negligible & \\
E - Improbable & & \\
\hline
\end{tabular}

Risk Designation

After

Applying

Mitigations:

\begin{tabular}{|lll|}
\hline Frequency Rating: & Consequence Rating: & Risk After: \\
OA - Frequent & $\bigcirc I$ - Catastrophic & \\
B - Probable & $\bigcirc I I-$ Critical & Extremely Low \\
C - Occasional & III- Marginal & \\
D-Remote & IV - Negligible & \\
E- Improbable & & \\
\hline
\end{tabular}

Related Scenarios:

U10206

Prevention

Mitigation

Documentation: 


\section{DI: BAB000000-01717-0200-0004; Rev 02}

\begin{tabular}{|c|c|}
\hline \multicolumn{2}{|r|}{ Mitigation Feature } \\
\hline Scenario Number: & Mitigation Number: | \\
\hline Scenario: & $\begin{array}{l}\text { Train/locomotive derailment at turnout (intersection of East-West Starter Tunnel and TS Main } \\
\text { Drift). }\end{array}$ \\
\hline Mitigation Feature: & JSAs, procedures and/or training will address hazards associated with train operations. \\
\hline Documentation: & $\begin{array}{l}\text { Inspection and maintenance records; training manuals; operators manuals; maintenance } \\
\text { manuals; safety manuals; operator training records. }\end{array}$ \\
\hline Remarks: & \\
\hline Mitigation type: & $\begin{array}{l}\bigcirc \text { Design Feature } \\
\text { Safety Device } \\
\text { Warning Device } \\
\text { Procedure } \\
\text { Training }\end{array}$ \\
\hline
\end{tabular}

\section{Mitigation Tracking}

Mitigation

Implementation

Status:

Comments:
(Not Implemented

Implemented 
DI: BAB000000-01717-0200-0004, Rev 02

\section{Scenario Description and Analysis}

\section{Approved}

Scenario Number:

Revision Number:

Revision Date:

Scenario:

Cause, Failure, or Hazardous Event:

Risk Assignment Before Considering Mitigations:

\section{After}

Risk Designation

Applying

Mitigations:

Related Scenarios:

Prevention

Mitigation

Documentation:

\begin{tabular}{|l|}
\hline$U 15047$ \\
\hline 02 \\
\hline $12 / 17 / 98$ \\
\hline
\end{tabular}

\author{
SSA: East-West Drift \\ Location: East-West Drift
}

Failure of emergency backup lighting; material spills; human error; lack of adequate illumination; debris (e.g., oil, water, tools) on surfaces; slippery surfaces; lack of non-skid surfaces; failure to adhere to safety procedures and rules.

\begin{tabular}{|lll|}
\hline Frequency Rating: & Consequence Rating: & *Risk Before: \\
A - Frequent & $O_{I-\text { Catastrophic }}$ & \\
B - Probable & $O_{I}$ - Critical & High \\
C - Occasional & $O I I I-$ Marginal & \\
D - Remote & $\bigcirc I V$ - Negligible & \\
E - Improbable & & \\
\hline
\end{tabular}

\begin{tabular}{|c|c|c|}
\hline Frequency Rating: & Consequence Rating: & Risk After: \\
\hline A - Frequent & 1. Catastrophic & \\
\hline B - Probable & OII - Critical & \\
\hline Oc - Occasional & $\bigcirc$ III - Marginal & Low \\
\hline OD-Remote & IV - Negligible & \\
\hline OE-Improbable & & \\
\hline
\end{tabular}

U10265, U10266, UI5069 (severe trips \& slips)

Inspection, maintenance and cleanup records; maintenance manuals; safety manuals 


\section{DI: BAB000000-01717-0200-0004, Rev 02}

\section{Mitigation Feature}

Scenario Number: $\quad$ U15047 Mitigation Number: 1

Scenario: $\quad$ General slips and trips resulting in minor personnel injury (see also U/5069).

Mitigation Feature: Provide adequate and proper illumination in the ECRB for the expected population and activities.

Documentation: Inspection, maintenance and cleanup records; maintenance manuals; safety manuals

Remarks:

Mitigation type:

Design Feature

Safety Device

Warning Device

Procedure

Training

\section{Mitigation Tracking}

Mitigation

Implementation

Status:

Comments:
Not Implemented

Implemented

Implemented (per T.Rotert, aug98.) wp

\section{Appendix A \\ Page UI5047.1}




\section{DI: BAB000000-01717-0200-0004; Rev 02}

\section{Mitigation Feature}

Scenario Number: $415047 \quad$ Mitigation Number: 2

$\begin{array}{ll}\text { Scenario: } & \text { General slips and trips resulting in minor personnel injury (see also U[5069). } \\ \text { Mitigation Feature: } & \text { Provide non-skid surfaces on platforms and walkways. } \\ \text { Documentation: } & \begin{array}{l}\text { Inspection, maintenance and cleanup records; design drawings; maintenance manuals; safety } \\ \text { manuals }\end{array} \\ \text { Remarks: } & \\ \text { Mitigation type: } & \text { Design Feature } \\ \text { Safety Device } \\ \text { Warning Device } \\ \text { Procedure } \\ \text { Training }\end{array}$

\section{Mitigation Tracking}

Mitigation

Implementation

Status:

Comments:
Not Implemented

Implemented

Implemented (per T.Rotert, aug98__Non-skid surfaces on mapping fioor, walkway and TBM.) wp

\section{Appendix A}

Page U15047.2 
DI: BABO00000-01717-0200-0004, Rev 02

\section{Mitigation Feature}

$\begin{array}{ll}\text { Scenario Number: } & \text { UI5047 } \\ \text { Scenario: } & \text { Meneral slips and trips resulting in minor personnel injury (see also UI5069). } \\ \text { Mitigation Feature: } & \begin{array}{l}\text { Provide safety training and caution all personnel regarding uneven surfaces on platforms and } \\ \text { tunnel flooring. }\end{array} \\ \text { Documentation: } & \begin{array}{l}\text { Inspection, maintenance and cleanup records; design drawings; maintenance manuals; safety } \\ \text { manuals }\end{array} \\ \text { Remarks: } & \\ \text { Mitigation type: } & \begin{array}{l}\text { Design Feature } \\ \text { Safety Device } \\ \text { Warning Device } \\ \text { Procedure } \\ \text { Training }\end{array} \\ & \end{array}$

\section{Mitigation Tracking}

Mitigation Implementation Status:

Comments:
Not Implemented

Implemented

Implemented (per T.Rotert, aug98_Covered in GUT training.) wp

\section{Appendix A}

Page UI5047.3 


\section{DI: BAB000000-01717-0200-0004; Rev 02}

\section{Mitigation Feature}

Scenario Number: $\quad$ UI5047 Mitigation Number: 4

Scenario:

General slips and trips resulting in minor personnel injury (see also UI5069).

Mitigation Feature: Establish inspection, maintenance and good housekeeping rules.

Documentation: Inspection, maintenance and cleanup records; maintenance manuals; safety manuals

Remarks:

Mitigation type:
Design Feature
Safety Device
Warning Device
Procedure
Training

\section{Mitigation Tracking}

Mitigation Implementation Status:

Comments:

\section{Not Implemented}

Implemented

Implemented (per T.Rotert, aug98.) wp

\section{Appendix A}

Page UI5047.4 
DI: BAB000000-01717-0200-0004, Rev 02

\section{Mitigation Feature}

$\begin{array}{ll}\text { Scenario Number: } & \text { UI5047 } \\ \text { Scenario: } & \text { General slips and trips resulting in minor personnel injury (see also UI5069). } \\ \text { Mitigation Feature: } & \text { Provide first-aid kits on the TBM and at the entrance to the ECRB. } \\ \text { Documentation: } & \begin{array}{l}\text { Inspection, maintenance and cleanup records; design drawings; maintenance manuals; safety } \\ \text { manuals }\end{array} \\ \text { Remarks: } & \\ \text { Mitigation type: } & \text { Design Feature } \\ \text { Safety Device } \\ \text { OWarning Device } \\ \text { Procedure } \\ \text { Training }\end{array}$

Mitigation Tracking

Mitigation Implementation

Status:

Implemented

Comments: $\quad$ Implemented (per T. Rotert, aug98) wp.

Appendix A

Page UI5047.5 


\section{DI: BAB000000-01717-0200-0004, Rev 02}

\section{Mitigation Feature}

Scenario Number:

U15047

Mitigation Number:

6

Scenario:

General slips and trips resulting in minor personnel injury (see also UI5069).

Mitigation Feature: Use personal lighting (i.e., cap lamps) in unlighted or poorly illuminated areas.

Documentation: Inspection, maintenance and cleanup records; maintenance manuals; safety manuals

Remarks:

Mitigation type:
Design Feature
Safety Device
Warning Device
Procedure
Training

\section{Mitigation Tracking}

Mitigation Implementation Status:

Comments:

Not Implemented
Implemented

Implemented (per T. Rotert, aug98) wp.

\section{Appendix A}

Page UI5047.6 


\section{Scenario Description and Analysis}

\section{Approved}

Scenario Number:

\begin{tabular}{|l|}
\hline UI5048 \\
\hline 02 \\
\hline $12 / 17 / 98$ \\
\hline
\end{tabular}

SSA: East-West Drift

Revision Number:

Revision Date:

Location: East-West Drift

Scenario:

Personnel injury due to tools, equipment, or debris dropping or falling from an elevated platform.

Cause, Failure, or Hazardous Event:

Human error; dropped tools or equipment; material spills; loss of balance; failure to adhere to JSA, safety procedures and rules.

Risk Assignment

Before Considering

Mitigations:

\begin{tabular}{|lll|}
\hline Frequency Rating: & Consequence Rating: & *Risk Before: \\
A - Frequent & I - Catastrophic & \\
B - Probable & II - Critical & Medium \\
C - Occasional & III - Marginal & \\
D - Remote & IV - Negligible & \\
E - Improbable & & \\
\hline
\end{tabular}

Risk Designation

After

Applying

Mitigations:
Frequency Rating:
Consequence Rating:
C A - Frequent
I - Catastrophic
O B - Probable
III - Critical
C - Occasional
III - Marginal
D - Remote
ON-Negligible
E - Improbable

Risk After:

Related Scenarios:

U10109

Prevention

Mitigation

Documentation:

Safety manual; JSA, OHSA - 29 CFR 1926 Subpart E; maintenance manuals 


\section{DI: ВAB000000-01717-0200-0004, Rev 02}

\section{Mitigation Feature}

Scenario Number: U15048 Mitigation Number:

Scenario: $\quad$ Personnel injury due to tools, equipment, or debris dropping or falling from an elevated platform.

Mitigation Feature: Control personnel and any ongoing work activities below an elevated platform in the ECRB.

Documentation: Safety manual; JSA; OHSA - 29 CFR 1926 Subpart E; maintenance manuals

Remarks:

Mitigation type:
$\bigcirc$ Design Feature
Safety Device
Warning Device
Procedure
Training

\section{Mitigation Tracking}

\section{Mitigation Implementation Status:}

Comments:
Not Implemented

Implemented

mplemented (per T. Rotert, aug98__Portal guard established and A/B/C badge system established) wp. 


\section{DI: BAB000000-01717-0200-0004, Rev 02}

\section{Mitigation Feature}

Scenario Number:

U15048 Mitigation Number:

Scenario:

Personnel injury due to tools, equipment, or debris dropping or falling from an elevated platform.

Mitigation Feature: Screen enclosure with toe plate around work platforms.

Documentation: Safety manual; JSA; OHSA - 29 CFR 1926 Subpart E; maintenance manuals

Remarks:

Mitigation type: Design Feature

Safety Device

Warning Device

Procedure

Training

\section{Mitigation Tracking}

Mitigation

Implementation

Status:

Not Implemented

Implemented

Comments:

Toe/kick plates are used on all elevated platfarms. Screens are not used. Bob Law 05/19/99 JLR.

Appendix A

Page UI5048.2 


\section{DI: BAB000000-01717-0200-0004; Rev 02}

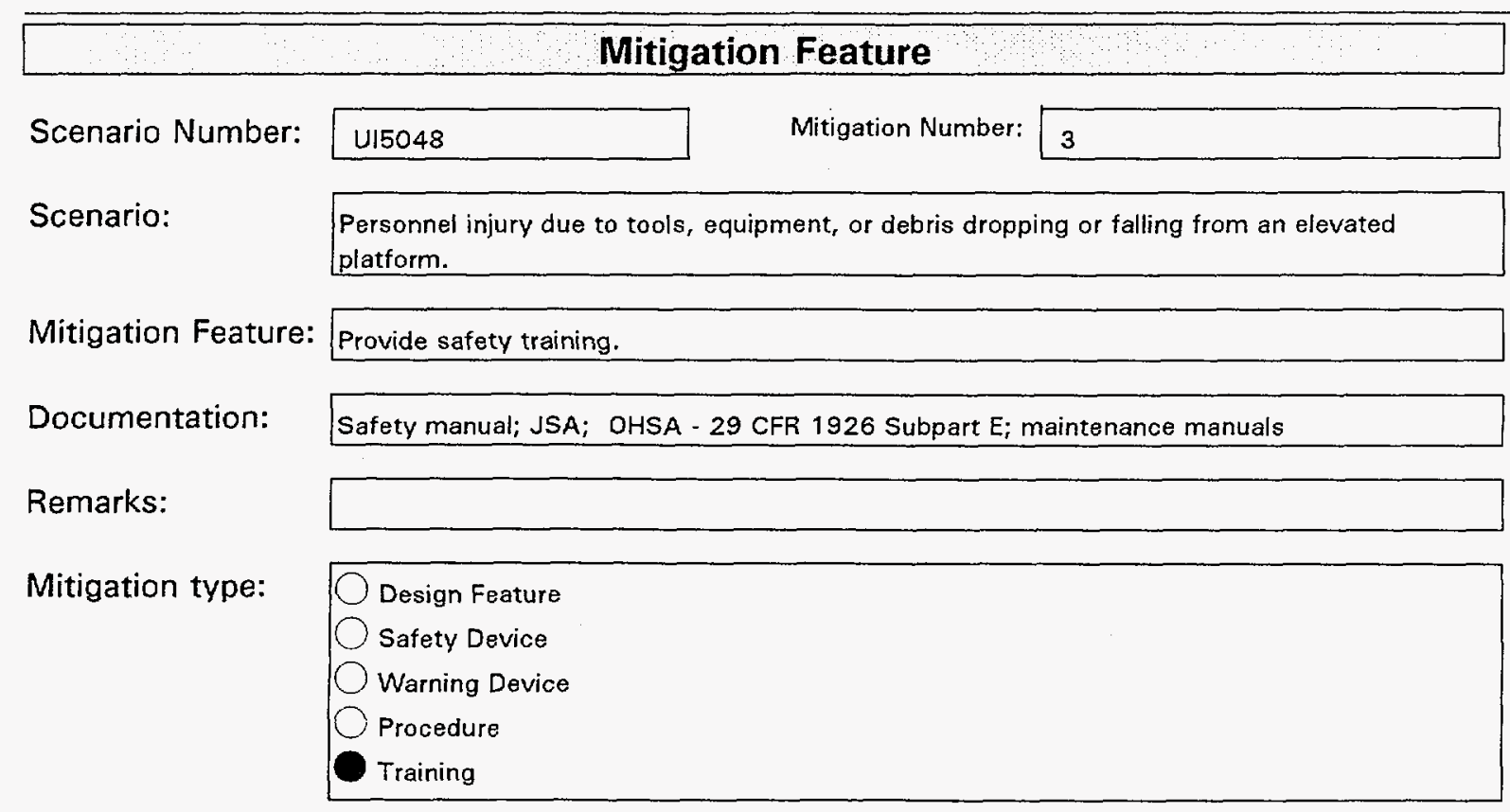

\section{Mitigation Tracking}

Mitigation

Implementation

Status:

Comments:

\section{Not Implemented \\ Implemented}

Implemented (per T.Rotert, aug98_Covered in GUT training.) wp 


\section{DI: BAB000000-01717-0200-0004, Rev 02}

\section{Mitigation Feature}

Scenario Number:

\section{U15048}

Mitigation Number:

4

Scenario:

Personnel injury due to tools, equipment, or debris dropping or falling from an elevated platform.

Mitigation Feature: Hardhats, safety glasses, and steel-toe shoes are required underground.

Documentation:

Safety manual; JSA; OHSA - 29 CFR 1926 Subpart E; maintenance manuals

Remarks:

Mitigation type:
Design Feature
Safety Device
Warning Device
Procedure
Training

\section{Mitigation Tracking}

Mitigation

Implementation

Status:

Comments:
Not Implemented

Implemented 


\section{DI: BAB000000-01717-0200-0004, Rev 02}

\section{Mitigation Feature}

Scenario Number:

U15048

5

Scenario:

Personnel injury due to tools, equipment, or debris dropping or falling from an elevated platform.

Mitigation Feature: Tools/equipment will be tethered to an individual or the platform, as appropriate.

Documentation: Safety manual; JSA; OHSA - 29 CFR 1926 Subpart E; maintenance manuals

Remarks:

Mitigation type:
Design Feature
Safety Device
Warning Device
Procedure

Training

\section{Mitigation Tracking}

Mitigation Implementation Status:

Comments:
Not Implemented

Implemented

Implemented (per T. Rotert, aug98) wp. 


\section{Scenario Description and Analysis}

\section{Approved}

Scenario Number:

Revision Number:

Revision Date:

Scenario:

Cause, Failure, or Hazardous Event:

Risk Assignment Before Considering Mitigations:

\section{Risk Designation}

After

Applying

Mitigations:

Related Scenarios:

Prevention

Mitigation

Documentation:

\begin{tabular}{|l|}
\hline U15050 \\
\hline 02 \\
\hline $12 / 17 / 98$ \\
\hline
\end{tabular}

SSA: East-West Drift

Location: East-West Drift

Personnel injury/equipment damage due to runaway vehicle le.g., train, locomotive, front-end loader).

Controls failure, human error, failure to adhere to safety procedures and rules.

\begin{tabular}{|llc|}
\hline Frequency Rating: & Consequence Rating: & *Risk Before: \\
A - Frequent & I - Catastrophic & \\
B - Probable & II - Critical & High \\
C - Occasional & III - Marginal & \\
D-Remote & $\bigcirc$ IV - Negligible & \\
E- Improbable & & \\
\hline
\end{tabular}

\begin{tabular}{|lll|}
\hline Frequency Rating: & Consequence Rating: & Risk After: \\
A - Frequent & I- Catastrophic & \\
B - Probable & II- Critical & Low \\
C - Occasional & OIII- Marginal & \\
D - Remote & IV - Negligible & \\
E - Improbable & & \\
\hline
\end{tabular}

U10041, U15046, U15067

Safety Manuals; inspection and maintenance records; vehicle inspections; OSHA - 29CFR

1926.600; operating procedures \& manuals; maintenance manuals.
Appendix A

Page UI5050 


\section{DI: BAB000000-01717-0200-0004; Rev 02}

\section{Mitigation Feature}

Scenario Number: U15050 Mitigation Number: 1

Scenario: $\quad$ Personnel injury/equipment damage due to runaway vehicle le.g., train, locomotive, front-end loader).

Mitigation Feature:

Provide restraints (e.g., chocks or blocks) on parked vehicles to prevent ralling down sloped grade.

Documentation: Safety Manuals; inspection and maintenance records; vehicle inspections; OSHA - 29CFR 1926.600; operating procedures \& manuals; maintenance schedules.

Remarks:

Mitigation type:
Design Feature
Safety Device
Warning Device
Procedure
Training

\section{Mitigation Tracking}

Mitigation Implementation

Status:

Comments:
Not Implemented

Implemented

Implemented (per T.Rotert, aug98_Loci carry stops and attachments are lowered on the drill and loader.) wp
Appendix A

Page UI5050.1 


\section{DI: BAB000000-01717-0200-0004; Rev 02}

\section{Mitigation Feature}

Scenario Number:

015050

Mitigation Number:

2

Scenario:

Personnel injury/equipment damage due to runaway vehicle (e.g., train, locomotive, front-end loader).

Mitigation Feature: Provide safety chains or cables between locomotive and cars and between cars.

Documentation: Safety Manuals; inspection and maintenance records; vehicle inspections; OSHA - 29CFR 1926.600; operating procedures \& manuals; maintenance manuals.

Remarks: Rev.01, Mit\#9

Mitigation type: $\bigcirc$ Design Feature

Safety Device

Warning Device

Procedure

Training

\section{Mitigation Tracking}

Mitigation Implementation

Status:

Not Implemented

Implemented

Comments:

Implemented (per T. Rotert, aug98_Cables vice chains provided) wp.

Appendix A

Page UI5050.2 


\section{DI: BAB000000-01717-0200-0004; Rev 02}

\section{Mitigation Feature}

Scenario Number: $\quad$ U15050 Mitigation Number: 3

Scenario:

Personnel injury/equipment damage due to runaway vehicle le.g., train, locomotive, front-end loader).

Mitigation Feature: Provide safety training for vehicle operators (e.g., vehicle operators cannot leave a vehicle until it is properly secured).

Documentation: Safety Manuals; inspection and maintenance records; vehicle inspections; OSHA - 29CFR 1926.600; operating procedures \& manuals; maintenance manuals.

Remarks:

Mitigation type:
Design Feature
Safety Device
Warning Device
Procedure
Training

\section{Mitigation Tracking}

Mitigation Implementation

Status:

Comments:
Not Implemented

Implemented

Implemented (per T.Rotert, aug98) wp.

\section{Appendix A}

Page UI5050.3 


\section{DI: BAB000000-01717-0200-0004, Rev 02}

\section{Mitigation Feature}

Scenario Number: U15050 Mitigation Number:

Scenario: $\quad$ Personnel injury/equipment damage due to runaway vehicle le.g., train, locomotive, front-end loaderl.

Mitigation Feature: Establish operation, inspection and maintenance schedule for vehicles.

Documentation: Safety Manuals; inspection and maintenance records; vehicle inspections; OSHA - 29CFR 1926.600; operating procedures \& manuals; maintenance manuals.

Remarks:

Mitigation type:
Design Feature
Safety Device
Warning Device
Procedure
Training

\section{Mitigation Tracking}

Mitigation Implementation Status:

Comments:
Not Implemented

Implemented

Implemented (per T. Rotert, aug98) wp. 


\section{DI: BAB000000-01717-0200-0004; Rev 02}

\section{Mitigation Feature}

Scenario Number:

U15050

Mitigation Number:

5

Scenario:

Personnel injury/equipment damage due to runaway vehicle (e.g., train, locomotive, front-end loader).

Mitigation Feature: Personnel will set derail devices and/or bumper blocks in their work areas in the ECRB.

Documentation: Safety Manuals; inspection and maintenance records; vehicle inspections; OSHA - 29CFR 1926.600; operating procedures \& manuals; maintenance manuals.

Remarks:

Mitigation type:
Design Feature
Safety Device
Warning Device
Procedure
Training

\section{Mitigation Tracking}

Mitigation

Implementation

Status:

Comments:
Not Implemented

Implemented

Implemented (per T. Rotert, aug98) wp.

\section{Appendix A}

Page UI5050.5 


\section{DI: BAB000000-01717-0200-0004; Rev 02}

\begin{tabular}{|c|c|}
\hline \multicolumn{2}{|r|}{ Mitigation Feature } \\
\hline Scenario Number: & Mitigation Number: \\
\hline Scenario: & $\begin{array}{l}\text { Personnel injury/equipment damage due to runaway vehicle (e.g., train, locomotive, front-end } \\
\text { loader). }\end{array}$ \\
\hline Mitigation Feature: & $\begin{array}{l}\text { For trains, provide couplers with locks which prevent cars and locomotive from being } \\
\text { accidentally uncoupled. }\end{array}$ \\
\hline Documentation: & $\begin{array}{l}\text { Safety Manuals; inspection and maintenance records; vehicle inspections; OSHA - 29CFR } \\
\text { 1926.600; operating procedures \& manuals; maintenance manuals. }\end{array}$ \\
\hline Remarks: & \\
\hline Mitigation type: & $\begin{array}{l}\bigcirc \text { Design Feature } \\
\text { Safety Device } \\
\bigcirc \text { Warning Device } \\
\bigcirc \text { Procedure } \\
\bigcirc \text { Training }\end{array}$ \\
\hline
\end{tabular}

\section{Mitigation Tracking}

Mitigation Implementation Status:

Comments:
Not Implemented

Implemented

Implemented (per T. Rotert, aug98 Safety cables between cars provided) wp.

\section{Appendix A}

Page UI5050.6 


\section{DI: BAB000000-01717-0200-0004; Rev 02}

\section{Mitigation Feature}

Scenario Number:

U15050 Mitigation Number: 7

Scenario:

Personnel injury/equipment damage due to runaway vehicle (e.g., train, locomotive, front-end loaderl.

Mitigation Feature: Provide redundant braking systems on locomotives.

Documentation: Safety Manuals; inspection and maintenance records; vehicle inspections; OSHA - 29CFR 1926.600; operating procedures \& manuals; maintenance manuals.

Remarks:

Mitigation type:

Design Feature

Safety Device

Warning Device

Procedure

Training

\section{Mitigation Tracking}

Mitigation Implementation

Status:

Comments:
Not Implemented

implemented

Implemented (per T.Rotert, aug98_ Air and mechanical systems.) wp 


\section{DI: BAB000000-01717-0200-0004; Rev 02}

\section{Mitigation Feature}

$\begin{array}{ll}\text { Scenario Number: } & \text { U15050 Mitigation Number: } \\ \text { Scenario: } & \begin{array}{l}\text { Personnel injury/equipment damage due to runaway vehicle (e.g., train, locomotive, front-end } \\ \text { loader). }\end{array} \\ \text { Mitigation Feature: } & \begin{array}{l}\text { Design locomotive operator console to include "deadman" controls and indicators for braking } \\ \text { system status and brake setting. }\end{array}\end{array}$

Documentation: Safety Manuals; inspection and maintenance records; vehicle inspections; OSHA - 29CFR 1926.600 ; operating procedures \& manuals; maintenance manuals.

Remarks:

Mitigation type:

\section{Mitigation Tracking}

Mitigation Implementation

Status:

Comments:
Design Feature

Safety Device

Warning Device

Procedure

Training
Not Implemented

Implemented

Implemented (per T.Rotert, aug98_Deadman is in place, operator is accompanied by the swamper.) wp

Appendix A

Page UI5050.8 


\section{Scenario Description and Analysis}

\section{Approved}

Scenario Number:

Revision Number:

Revision Date:

Scenario:

Cause, Failure, or Hazardous Event:

Risk Assignment Before Considering Mitigations:

\begin{tabular}{l} 
U15054 \\
\hline $12 / 17 / 98$ \\
\hline
\end{tabular}

SSA

Location: East-West Drift hat or eye protection); vehicle collision with tunnel wall/steel sets; earthquake.

Failure of ground support system; failure to adhere to safety procedures and rules (e.g., hard

\begin{tabular}{|lll|}
\hline Frequency Rating: & Consequence Rating: & *Risk Before: \\
A - Frequent & I-Catestrophic & \\
B - Probable & II - Critical & High \\
C - Occasional & III - Marginal & \\
D - Remote & IV - Negligible & \\
E - Improbable & & \\
\hline
\end{tabular}

Risk Designation

After

Applying

Mitigations:

\begin{tabular}{|ll}
\hline Frequency Rating: & Consequence Rating: \\
A - Frequent & I- Catastrophic \\
B - Probable & II - Critical \\
C - Occasional & $O I I$ - Marginal \\
D- Remote & OIV - Negligible \\
E- Improbable &
\end{tabular}

Risk After:

Extremely Low

Related Scenarios:

U10271, U10360

Prevention

Mitigation

Documentation:

Training manuals; safety manuals; emergency evacuation plan/procedure; test/inspection/maintenance records; OSHA - 29 CFR 1926 Subpart S; ECRB Starter Tunnel and Starter Tunnel/North Ramp Intersection Stability Analysis,

BABEE0000-01717-0200-00016 Rev 00

\section{Appendix A}

Page UI5054 


\section{DI: BAB000000-01717-0200-0004; Rev 02}

\section{Mitigation Feature}

Scenario Number:

U15054 Mitigation Number: 1

Scenario:

Personnel injury due to failure of ground support (e.g., rock fall).

Mitigation Feature: Implement JSAs for drill \& blast and/or mechanical excavation.

Documentation: Training manuals; safety manuals; emergency evacuation plan/procedure;

test/inspection/maintenance records; OSHA - 29 CFR 1926 Subpart S; ECRB Starter Tunnel and Starter Tunnel/North Ramp Intersection Stability Analysis,

BABEE0000-01717-0200-00016 Rev 00

Remarks:

Mitigation type:
Design Feature
Safety Device
$\bigcirc$ Warning Device
Procedure
Training

\section{Mitigation Tracking}

Mitigation Implementation Status:

Not Implemented

Impiemented

\section{Comments:}

\section{Appendix A}

Page UI5054.1 


\section{Dl: BAB000000-01717-0200-0004, Rev 02}

\section{Mitigation Feature}

Scenario Number: $\quad$ LI5054 Mitigation Number: 2

Scenario: $\quad$ Personnel injury due to failure of ground support (e.g., rock fall).

Mitigation Feature: Test and inspect rockbolts and shotcrete. ...

Documentation: Training manuals; safety manuals; emergency evacuation plan/procedure;

test/inspection/maintenance records; OSHA - 29 CFR 1926 Subpart S; ECRB Starter Tunnel and Starter Tunnel/North Ramp Intersection Stability Analysis,

BABEEODO0-01717-0200-00016 Rev 00

Remarks:

Mitigation type:
Design Feature
Safety Device
Warning Device
Procedure
Training

\section{Mitigation Tracking}

\section{Mitigation Implementation Status:}

Comments:
Not Implemented

Implemented

Implemented (per T.Rotert, aug98_. Tested prior to use.) wp

\section{Appendix A}

Page UI5054.2 


\section{DI: BAB000000-01717-0200-0004; Rev 02}

\section{Mitigation Feature}

$\begin{array}{ll}\text { Scenario Number: } & \text { U15054 } \\ \text { Scenario: } & \text { Mersonnel injury due to failure of ground support (e.g., rock fall). } \\ \text { Mitigation Feature: } & \text { Provide personnel safety training. } \\ \text { Documentation: } & \begin{array}{l}\text { Training manuals; safety manuals; emergency evacuation plan/procedure; } \\ \text { test/inspection/maintenance records; OSHA - 29 CFR 1926 Subpart S; ECRB Starter Tunnel } \\ \text { and Starter Tunnet/North Ramp Intersection Stability Analysis, } \\ \text { BABEEO000-01717-0200-00016 Rev 00 }\end{array}\end{array}$

Remarks:

Mitigation type:
Design Feature
Safety Device
Warning Device
$\bigcirc$ Procedure
Training

\section{Mitigation Tracking}

Mitigation Implementation Status: Not Implemented

Implemented

Comments: 


\section{DI: BAB000000-01717-0200-0004, Rev 02}

\section{Mitigation Feature}

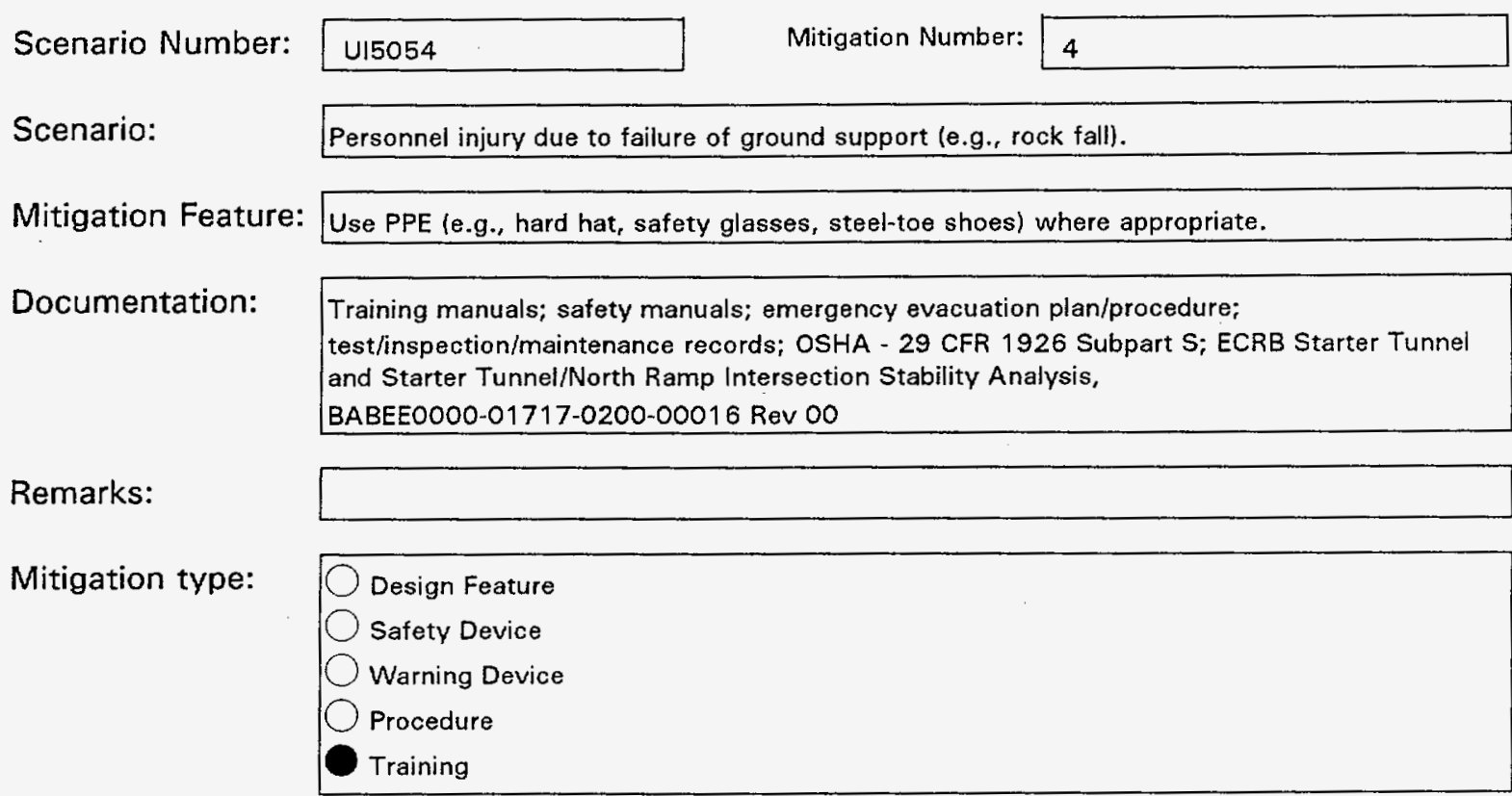

\section{Mitigation Tracking}

Mitigation Implementation Status:

Comments: $\quad$ Implemented (per T.Rotert, aug98.) wp

Not Implemented

Implemented

Appendix A

Page UI5054.4 


\section{DI: BAB000000-01717-0200-0004; Rev 02}

\section{Mitigation Feature}

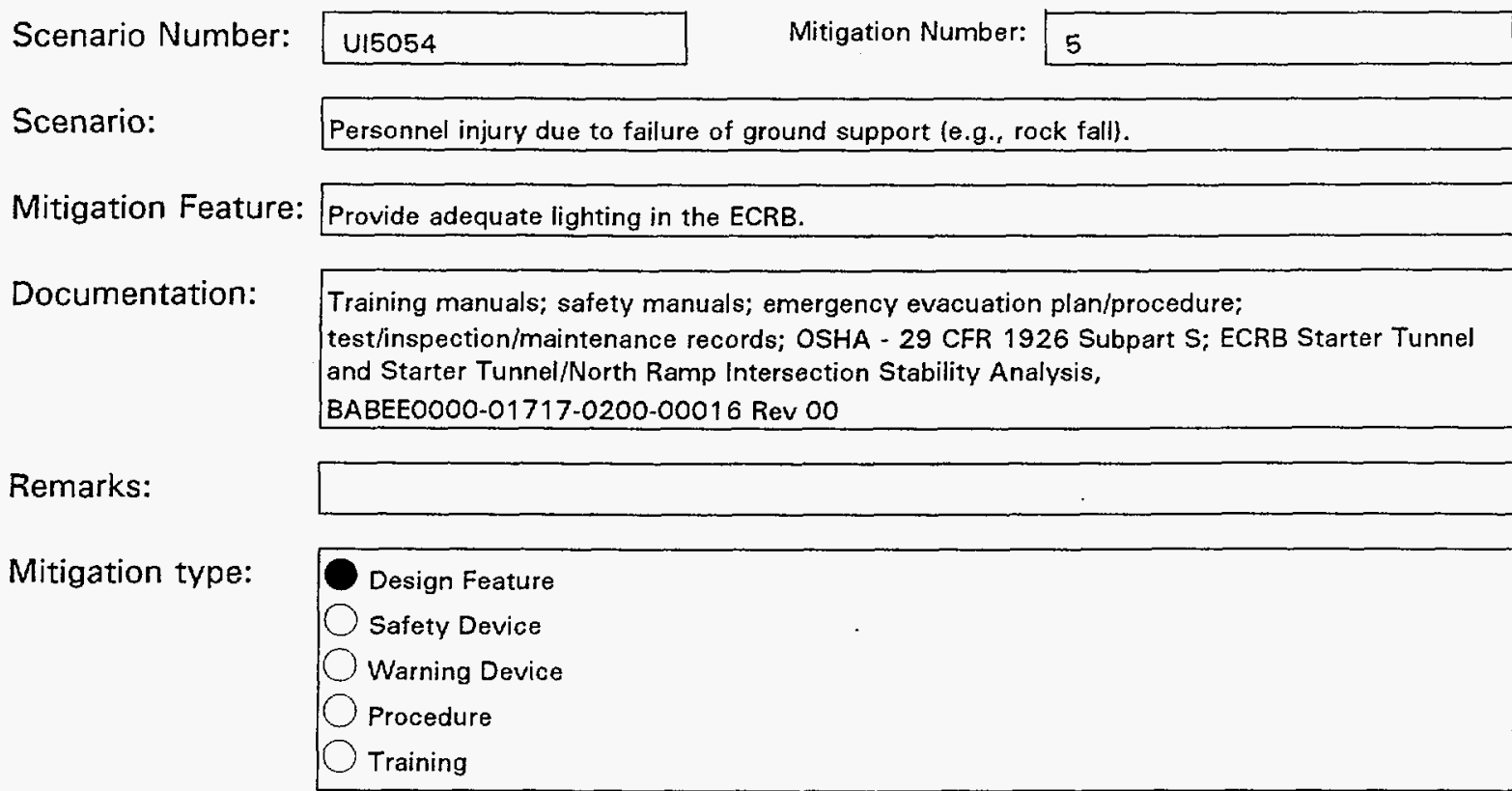

\section{Mitigation Tracking}

Mitigation

Implementation

Status:

Not Implemented

Implemented

Comments:

Implemented (per T.Rotert, aug98.) wp

Appendix A

Page UI5054.5 


\section{DI: BAB000000-01717-0200-0004; Rev 02}

\section{Mitigation Feature}

$\begin{array}{ll}\text { Scenario Number: } & \text { U15054 } \\ \text { Scenario: } & \text { Personnel injury due to failure of ground support (e.g., rock fall). } \\ \text { Mitigation Feature: } & \begin{array}{l}\text { Steel sets, lagging, rock bolts, wire mesh and shotcrete are installed based on analysis of } \\ \text { ground type and condition and location of faults. }\end{array} \\ \text { Documentation: } & \begin{array}{l}\text { Training manuals; safety manuals; emergency evacuation plan/procedure; } \\ \text { test/inspection/maintenance records; OSHA - 29 CFR 1926 Subpart S; ECRB Starter Tunnel } \\ \text { and Starter Tunnel/North Ramp Intersection Stability Analysis, } \\ \text { BABEEOOOO-01717-0200-00016 Rev OO }\end{array}\end{array}$

Remarks:

Mitigation type:
$\bigcirc$ Design Feature
Safety Device
Warning Device
Procedure
Training

\section{Mitigation Tracking}

Mitigation Implementation Status:

Comments:

\section{Not Implemented \\ Implemented}

Implemented (per T.Rotert, aug98.) wp

\section{Appendix A}

Page UI5054.6 


\section{DI: BAB000000-01717-0200-0004; Rev 02}

\section{Mitigation Feature}

Scenario Number: U15054 Mitigation Number: 7

Scenario:

Personnel injury due to failure of ground support (e.g., rock fall).

Mitigation Feature: Implement emergency evacuation plan.

Documentation: Training manuals; safety manuals; emergency evacuation plan/procedure; test/inspection/maintenance records; OSHA - 29 CFR 1926 Subpart S; ECRB Starter Tunnel and Starter Tunnel/North Ramp Intersection Stability Analysis,

BABEEO000-01717-0200-00016 Rev 00

Remarks:

Mitigation type:
Design Feature
Safety Device
Warning Device
Procedure
Training

\section{Mitigation Tracking}

\section{Mitigation Implementation Status:}
Not Implemented
Implemented

Comments:

\section{Appendix A \\ Page UI5054.7}




\section{DI: BAB000000-01717-0200-0004; Rev 02}

\section{Mitigation Feature}

Scenario Number: $\quad$ Ui5054 Mitigation Number: 8

Scenario:

Personnel injury due to failure of ground support (e.g., rock fall).

Mitigation Feature: Establish vehicle safety inspection and maintenance procedures and schedule.

Documentation: Training manuals; safety manuals; emergency evacuation plan/procedure; test/inspection/maintenance records; OSHA - 29 CFR 1926 Subpart S; ECRB Starter Tunnel and Starter Tunnel/North Ramp Intersection Stability Analysis,

BABEEO000-01717-0200-00016 Rev 00

Remarks:

Mitigation type:
$\bigcirc$ Design Feature
Safety Device
Warning Device
Procedure
Training

\section{Mitigation Tracking}

Mitigation Implementation Status:

Comments:
Not Implemented

Implemented

\section{Appendix A}

Page UI5054.8 


\section{Scenario Description and Analysis}

\section{Approved}

Scenario Number:

Revision Number:

Revision Date:

Scenario:

Cause, Failure, or Hazardous Event:

Risk Assignment Before Considering Mitigations:

\begin{tabular}{|l|}
\hline U15057 \\
\hline 02 \\
\hline $12 / 17 / 98$ \\
\hline
\end{tabular}

General underground fire due to ignition of trash, solvents, chemicals, or other combustible product.

Electrical cables, connections (splices), outlets sparking in the presence of hydraulic fluid, lubricating oil, diesel fuel, cleaning solvents, disposable cleanup materials, or catalytic reactions involving the above materials; lack of storage space/facilities.

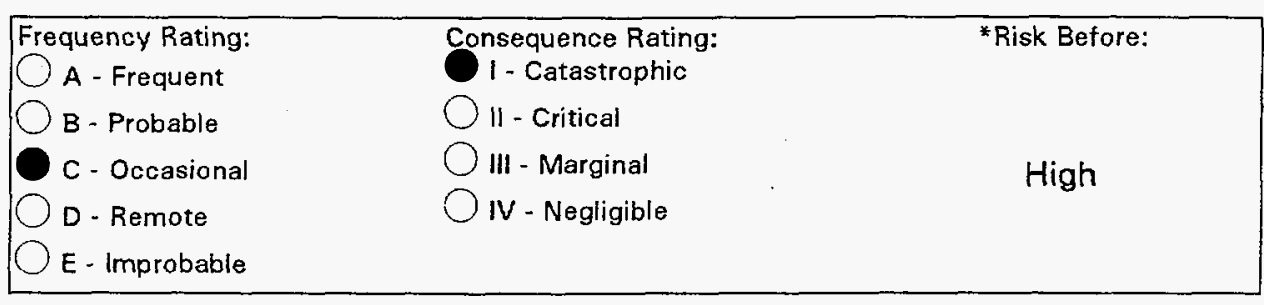

Risk Designation

After

Applying

Mitigations:

\begin{tabular}{|lll|}
\hline Frequency Rating: & Consequence Rating: & Risk After: \\
A - Frequent & I- Catastrophic & \\
B - Probable & II - Critical & Extremely Low \\
C - Occasional & III- Marginal & \\
D-Remote & OIV - Negligible & \\
E- Improbable & & \\
\hline
\end{tabular}

Related Scenarios:

U10056, U10053

Prevention

Mitigation

Documentation:
Safety Manuals; OSHA - 29 CFR 1926 Subpart S; NFPA Fire Protection Rules and Regulations

\section{Appendix A \\ Page UI5057}




\section{DI: BAB000000-01717-0200-0004; Rev 02}

\section{Mitigation Feature}

Scenario Number: $\quad$ U15057 Mitigation Number: 1

Scenario:

General underground fire due to ignition of trash, solvents, chemicals, or other combustible product.

Mitigation Feature: Implement JSAs/procedures for handling or working with combustible materials.

Documentation: Safety Manuals; OSHA - 29 CFR 1926 Subpart S; NFPA Fire Protection Rules and Regulations

Remarks:

Mitigation type: $\bigcirc$ Design Feature

Safety Device

Warning Device

Procedure

Training

\section{Mitigation Tracking}

Mitigation Implementation Status:

Comments:
Not Implemented

Implemented

Implemented (per T.Rotert, aug98_Storage of combustibles according to OSHA in JSA procedures.) wp
Appendix A

Page UI5057.1 


\section{DI: BAB000000-01717-0200-0004; Rev 02}

\section{Mitigation Feature}

Scenario Number: 415057 Mitigation Number: 2

Scenario:

General underground fire due to ignition of trash, solvents, chemicals, or other combustible product.

Mitigation Feature: Minimize use of combustible materials underground.

Documentation: Safety Manuals; OSHA - 29 CFR 1926 Subpart S; NFPA Fire Protection Rules and Regulations

Remarks:

Mitigation type:
Design Feature
Safety Device
Warning Device
Procedure
Oraining

\section{Mitigation Tracking}

Mitigation Implementation

Status:

Comments:
Not Implemented

Implemented

Appendix A

Page UI5057.2 


\section{DI: BAB000000-01717-0200-0004, Rev 02}

\section{Mitigation Feature}

Scenario Number: $\quad$ U15057 Mitigation Number: 3

Scenario: $\quad$ General underground fire due to ignition of trash, solvents, chemicals, or other combustible product.

Mitigation Feature: "Weld, cut and burn" procedures in effect for all underground areas.

Documentation: Safety Manuals; OSHA - 29 CFR 1926 Subpart S; NFPA Fire Protection Rules and Regulations

Remarks:

Mitigation type: $\quad$ Design Feature

Safety Device

Warning Device

Pracedure

Training

\section{Mitigation Tracking}

Mitigation

Implementation

Status:

Not implemented

Implemented

Comments:

Appendix A

Page UI5057.3 


\section{DI: BAB000000-01717-0200-0004; Rev 02}

\section{Mitigation Feature}

Scenario Number: $\quad$ U15057 Mitigation Number: 4

Scenario:

General underground fire due to ignition of trash, solvents, chemicals, or other combustible product.

Mitigation Feature: Use fire-resistant materials whenever practical.

Documentation: Safety Manuals; OSHA - 29 CFR 1926 Subpart S; NFPA Fire Protection Rules and Regulations

Remarks:

Mitigation type:
Design Feature
Safety Device
Warning Device
Procedure
Training

\section{Mitigation Tracking}

Mitigation

Implementation

Status:

Comments:
Not Implemented

implemented

Implemented (per T.Rotert, aug98.) wp

\section{Appendix A}

Page U15057.4 
DI: BAB000000-01717-0200-0004; Rev 02

\begin{tabular}{|c|c|c|}
\hline \multicolumn{3}{|c|}{ Mitigation Feature } \\
\hline Scenario Number: & Mitigation Number: & 5 \\
\hline Scenario: & $\begin{array}{l}\text { General underground fire due to ignition of trash, solvents } \\
\text { product. }\end{array}$ & chemicals, or other combustible \\
\hline Mitigation Feature: & Provide approved storage containers. & \\
\hline Documentation: & Safety Manuals; OSHA - 29 CFR 1926 Subpart S; NFPA & Fire Protection Rules and Regulations \\
\hline Remarks: & & \\
\hline Mitigation type: & $\begin{array}{l}\text { Design Feature } \\
\text { Safety Device } \\
\text { Warning Device } \\
\bigcirc \text { Procedure } \\
\text { Training }\end{array}$ & \\
\hline
\end{tabular}

\section{Mitigation Tracking}

Mitigation

Implementation

Status:

Comments:
Not Implemented

Implemented

Implemented (per T.Rotert, aug98.) wp

\section{Appendix A}

Page UI5057.5 
DI: BAB000000-01717-0200-0004; Rev 02

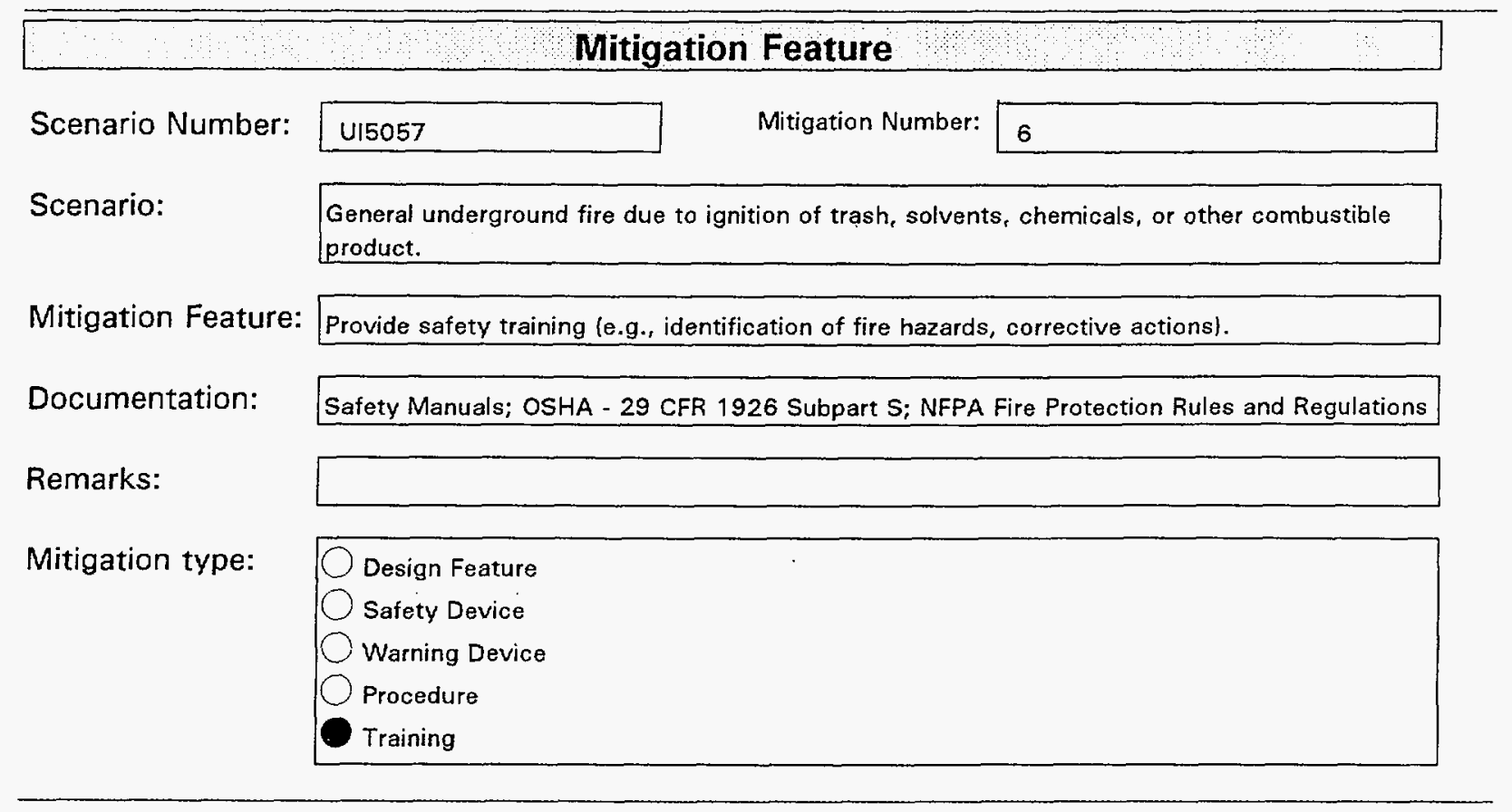

\section{Mitigation Tracking}

Mitigation Implementation

Status:

Comments:
Not Implemented

Implemented

Implemented (per T.Rotert, aug98 Covered in GUT training.) wp

Appendix A

Page UI5057.6 


\section{DI: BAB000000-01717-0200-0004, Rev 02}

\section{Mitigation Feature}

Scenario Number: $\quad$ UI5057 Mitigation Number: 7

Scenario:

General underground fire due to ignition of trash, solvents, chemicals, or other combustible product.

Mitigation Feature: Install portable extinguishers and hose bibs in the ECRB.

Documentation: $\quad$ Safety Manuals; OSHA - 29 CFR 1926 Subpart S; NFPA Fire Protectian Rules and Regulations

Remarks:

Mitigation type:
Design Feature
Safety Device
Warning Device
Procedure
Training

\section{Mitigation Tracking}

\section{Mitigation Implementation Status:}

Comments:
Not Implemented

Implemented

Implemented (per T.Rotert, aug98.) wp

\section{Appendix A}

Page U15057.7 


\section{DI: BAB000000-01717-0200-0004; Rev 02}

\section{Mitigation Feature}

Scenario Number:

U15057 Mitigation Number:

8

Scenario:

General underground fire due to ignition of trash, solvents, chemicals, or other combustible product.

Mitigation Feature: Limit diesel fuel storage volume to no more than 24-hour supply.

Documentation:

Safety Manuals; OSHA - 29 CFR 1926 Subpart S; NFPA Fire Protection Rules and Regulations

Remarks:

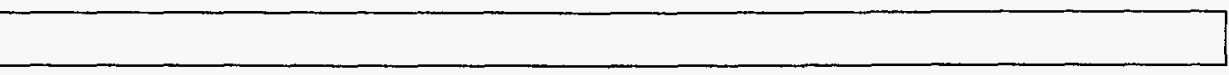

\begin{tabular}{l|l} 
Mitigation type: & Design Feature \\
Safety Device \\
Warning Device \\
Procedure \\
Training
\end{tabular}

\section{Mitigation Tracking}

Mitigation Implementation

Status:

Comments:
Not Implemented

Implemented

Implemented (per T.Rotert, aug98.) wp

\section{Appendix A}

Page UI5057.8 


\section{DI: BAB000000-01717-0200-0004; Rev 02}

\section{Mitigation Feature}

Scenario Number:

415057

Mitigation Number:

9

Scenario:

General underground fire due to ignition of trash, solvents, chemicals, or other combustible product.

Mitigation Feature: Implement good "house keeping" in the subsurface.

Documentation: Safety Manuals; OSHA - 29 CFR 1926 Subpart S; NFPA Fire Protection Rules and Regulations

Remarks:

Mitigation type:
Design Feature
Safety Device
Warning Device
Procedure
Training

\section{Mitigation Tracking}

Mitigation Implementation

Status:

Comments:
Not Implemented

Implemented

Implemented (per T.Rotert, aug98 Sanitation procedures implemented.) wp

\section{Appendix A \\ Page UI5057.9}




\section{DI: BAB000000-01717-0200-0004, Rev 02}

\section{Mitigation Feature}

Scenario Number:

U15057

Mitigation Number:

10

Scenario:

General underground fire due to ignition of trash, solvents, chemicals, or other combustible product.

Mitigation Feature: Use self rescuer as required.

Documentation: Safety Manuals; OSHA - 29 CFR 1926 Subpart S; NFPA Fire Protection Rules and Regulations

Remarks:

Mitigation type:
Design Feature
Safety Device
Warning Device
Procedure
Training

\section{Mitigation Tracking}

\section{Mitigation Implementation Status:}

Comments:

\section{Not Implemented}

Implemented

Implemented (per T.Rotert, aug98.) wp

\section{Appendix A}

Page UI5057.10 
DI: BAB000000-01717-0200-0004, Rev 02

\section{Mitigation Feature}

Scenario Number: $\quad$ U15057 Mitigation Number: 11

Scenario:

General underground fire due to ignition of trash, solvents, chemicals, or other combustible product.

Mitigation Feature: Ventilation system.

Documentation: Safety Manuals; OSHA - 29 CFR 1926 Subpart S; NFPA Fire Protection Rules and Regulations

Remarks:

Mitigation type:

Design Feature

$\bigcirc$ Safety Device

Warning Device

Procedure

0 Training

\section{Mitigation Tracking}

Mitigation

Implementation

Status:

Comments:
Not Implemented

Implemented 


\section{DI: BAB000000-01717-0200-0004; Rev 02}

\begin{tabular}{|c|c|}
\hline \multicolumn{2}{|r|}{ Mitigation Feature } \\
\hline Scenario Number: & Mitigation Number: \\
\hline Scenario: & $\begin{array}{l}\text { General underground fire due to ignition of trash, solvents, chemicals, or other combustible } \\
\text { product. }\end{array}$ \\
\hline Mitigation Feature: & Implement emergency evacuation plan. \\
\hline Documentation: & Safety Manuals; OSHA - 29 CFR 1926 Subpart S; NFPA Fire Protection Rules and Regulations \\
\hline Remarks: & \\
\hline Mitigation type: & $\begin{array}{l}\text { Design Feature } \\
\text { Safety Device } \\
\text { Warning Device } \\
\text { Procedure } \\
\text { Training }\end{array}$ \\
\hline
\end{tabular}

\section{Mitigation Tracking}

Mitigation

Implementation

Status:

Comments:
Not implemented

Implemented 


\section{Scenario Description and Analysis}

\section{Approved}

Scenario Number:

Revision Number:

Revision Date:

\begin{tabular}{|l|}
\hline$U 15058$ \\
\hline 02 \\
\hline $12 / 17 / 98$ \\
\hline
\end{tabular}

SSA: East-West Drift

Location: East-West Drift

\section{Scenario: \\ Cause, Failure, or Hazardous Event:}

Electrical shock resulting in personnel injury.

Personnel exposure to energized conductors, high voltage cables or static charges; electrical panel/transformer short circuit; defective electrical outlet; malfunctioning fuses/breakers; insulation failure; cable break; electrical overload

Risk Assignment Before Considering Mitigations:

\begin{tabular}{|lll|}
\hline Frequency Rating: & Consequence Rating: & *Risk Before: \\
A - Frequent & $O_{I-\text { Catastrophic }}$ & \\
B - Probable & $O_{I}$ - Critical & Medium \\
C - Occasional & $O_{N}$ - Marginal & \\
D-Regligible & \\
E- Improbable & & \\
\hline
\end{tabular}

Risk Designation

After

Applying

Mitigations:

\begin{tabular}{|lll|}
\hline Frequency Rating: & Consequence Rating: & Risk After: \\
A - Frequent & $O_{\text {I- Catastrophic }}$ & \\
B - Probable & II-Critical & Low \\
C - Occasional & III- Marginal & \\
D - Remote & IV - Negligible & \\
E - Improbable & & \\
\hline
\end{tabular}

Related Scenarios:

\section{$\mathrm{U} 10274$}

Prevention

Mitigation

Documentation:

Procedures, system specifications, Title II design drawings, maintenance manuals, training manuals, safety manual, electrician's certifications on file 


\section{DI: BAB000000-01717-0200-0004, Rev 02}

\section{Mitigation Feature}

$\begin{array}{ll}\text { Scenario Number: } & \text { Ul5058 } \\ \text { Scenario: } & \text { Mlectrical shock resulting in personnel injury. } \\ \text { Mitigation Feature: } & \text { Design electrical systems/components to applicable codes \& standards. } \\ \text { Documentation: } & \begin{array}{l}\text { Procedures, system specifications, Title II design drawings, maintenance manuals, training } \\ \text { manuals, safety manual }\end{array} \\ \text { Remarks: } & \\ \text { Mitigation type: } & \text { Design Feature } \\ & \text { Safety Device } \\ \text { Warning Device } \\ \text { Procedure } \\ \text { Training }\end{array}$

\section{Mitigation Tracking}

Mitigation

Implementation

Status:

Comments: $\quad$ Implemented (per T.Rotert, aug98.) wp

Not Implemented

Implemented

Appendix A

Page UI5058.1 


\section{DI: BAB000000-01717-0200-0004; Rev 02}

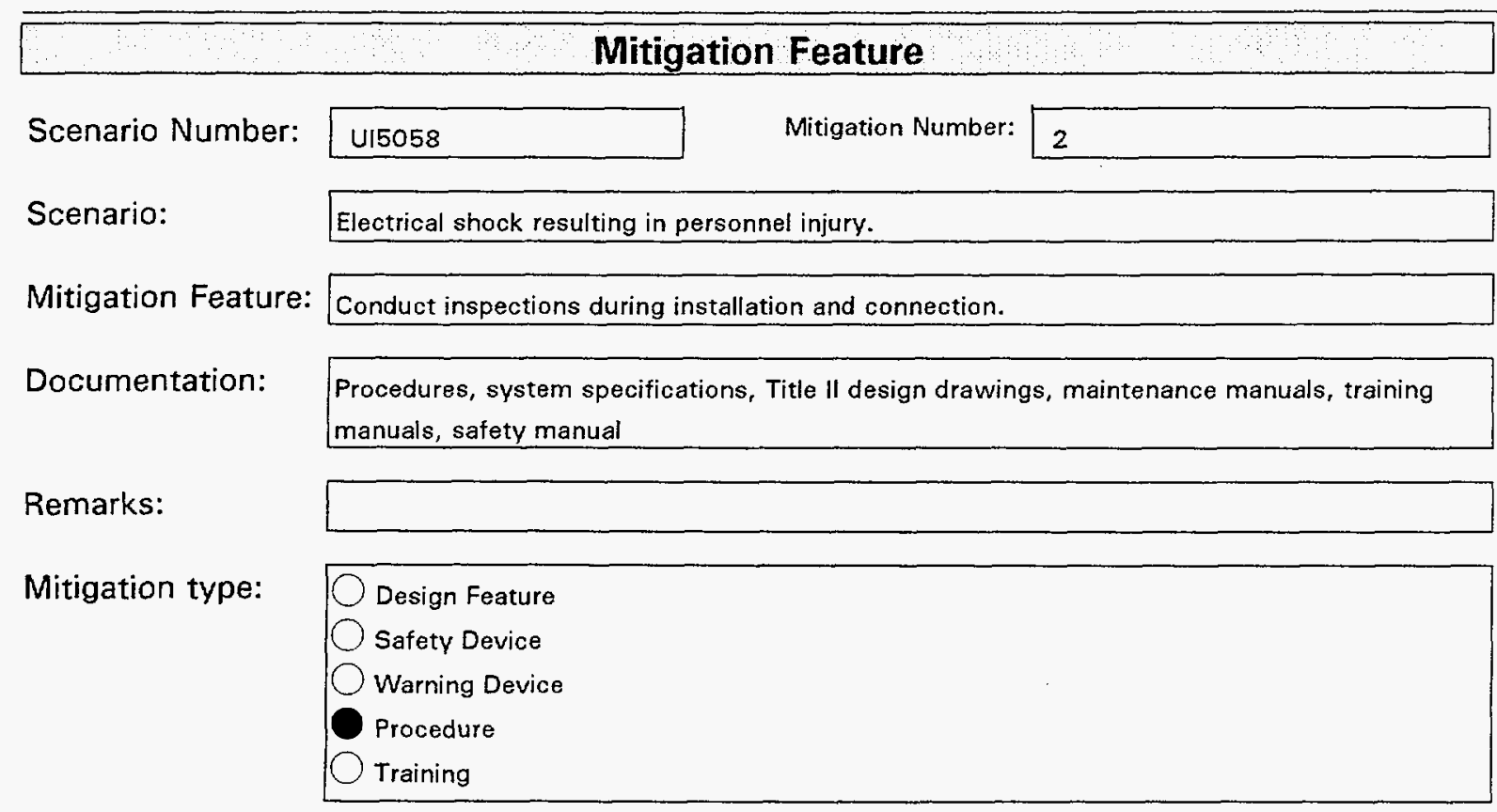

\section{Mitigation Tracking}

Mitigation Implementation Status:

Not Implemented

Implemented

Comments:

Implemented (per T.Rotert, aug98_Inspections conducted by A/E and QA.) wp

Appendix A

Page U15058.2 


\section{DI: BAB000000-01717-0200-0004; Rev 02}

\begin{tabular}{|c|c|}
\hline \multicolumn{2}{|r|}{ Mitigation Feature } \\
\hline Scenario Number: & Mitigation Number: \\
\hline Scenario: & Electrical shock resulting in personnel injury. \\
\hline Mitigation Feature: & Incorporate protective electrical devices where practical (e.g., fuses, circuit breakers). \\
\hline Documentation: & $\begin{array}{l}\text { Procedures, system specifications, Title II design drawings, maintenance manuals, training } \\
\text { manuals, safety manual }\end{array}$ \\
\hline Remarks: & \\
\hline Mitigation type: & $\begin{array}{l}\text { Design Feature } \\
\text { Safety Device } \\
\text { warning Device } \\
\text { Procedure } \\
\text { Training }\end{array}$ \\
\hline
\end{tabular}

\section{Mitigation Tracking}

Mitigation Implementation Status:

Comments:

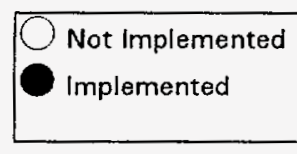

Implemented (per T.Rotert, aug98.) wp

\section{Appendix A}

Page UI5058.3 


\section{DI: BAB000000-01717-0200-0004; Rev 02}

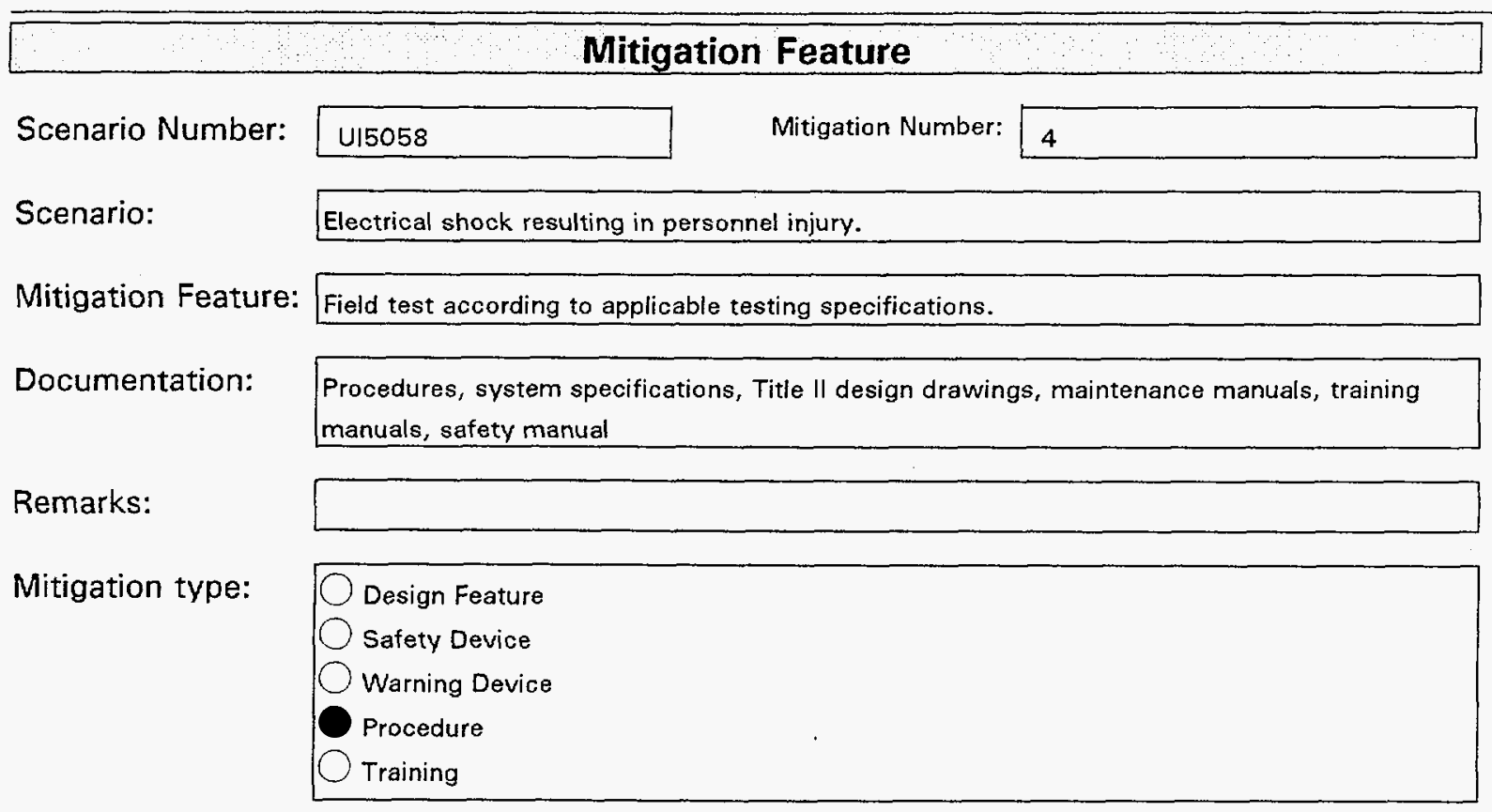

\section{Mitigation Tracking}

Mitigation

Implementation

Status:

Not Implemented

Implemented

Comments: 


\section{DI: BAB000000-01717-0200-0004, Rev 02}

\section{Mitigation Feature}

$\begin{array}{ll}\text { Scenario Number: } & \text { Ul5058 } \\ \text { Scenario: } & \text { Mlectrical shock resuiting in personnel injury. } \\ \text { Mitigation Feature: } & \text { Safety training for personnel working around electrical equipment. } \\ \text { Documentation: } & \begin{array}{l}\text { Procedures, system specifications, Title II design drawings, maintenance manuals, training } \\ \text { manuals, safety manual }\end{array} \\ \text { Remarks: } & \\ \text { Mitigation type: } & \text { Design Feature } \\ & \text { Safety Device } \\ & \text { Warning Device } \\ & \text { Procedure } \\ & \text { Training }\end{array}$

\section{Mitigation Tracking}

Mitigation Implementation

Status:

Comments:
Not Implemented

Implemented

Appendix A

Page UI5058.5 


\section{DI: BAB000000-01717-0200-0004, Rev 02}

\section{Scenario Description and Analysis}

\section{Approved}

Scenario Number:

Revision Number:

Revision Date:

\begin{tabular}{|l|}
\hline$U 15060$ \\
\hline 02 \\
\hline $12 / 17 / 98$ \\
\hline
\end{tabular}

SSA: East-West Drift

Air Quality - Excessive dust results in a personnel health hazard (see also U15017 and Ui5070).

\section{Scenario:}

Ventilation system failure or out-of-tolerance condition, ventilation duct leak, loss of off-site

Cause, Failure, or Hazardous Event: power

Risk Assignment Before Considering Mitigations:

\begin{tabular}{|lll|}
\hline Frequency Rating: & Consequence Rating: & *Risk Before: \\
A - Frequent & $\bigcirc I$ - Catastrophic & \\
B - Probable & $\bigcirc I I$ - Critical & High \\
C - Occasional & $\bigcirc I I$ - Marginal & \\
D - Remote & $\bigcirc I V$ - Negligible & \\
E- Improbable & & \\
\hline
\end{tabular}

Risk Designation

After

Applying

\begin{tabular}{|lll|}
\hline Frequency Rating: & Consequence Rating: & Risk After: \\
A - Frequent & $O_{1-\text { - Catastrophic }}$ & \\
B- Probable & $\bigcirc$ & \\
C - Occasional & $\bigcirc$ & Low - Marginal \\
D- Remote & IV- Negligible & \\
E- Improbable & & \\
\hline
\end{tabular}

Related Scenarios:

U15017, U15070

Prevention

Mitigation

Documentation:

Procedures, JSA(s), safety manuals

\section{Appendix A \\ Page UI5060}


DI: BAB000000-01717-0200-0004, Rev 02

\section{Mitigation Feature}

Scenario Number: 415060 Mitigation Number: 1

Scenario:

Air Quality - Excessive dust results in a personnel health hazard (see also UI5017 and Ui5070).

Mitigation Feature: Ventilation system.

Documentation: Procedures, JSA(s), safety manuals

Remarks:

Mitigation type:

Design Feature

Safety Device

Warning Device

Procedure

Training

\section{Mitigation Tracking}

Mitigation

Implementation

Status:

Comments:
Not Implemented

implemented 


\section{DI: BAB000000-01717-0200-0004, Rev 02}

\section{Mitigation Feature}

$\begin{array}{ll}\text { Scenario Number: } & \text { Ui5060 } \\ \text { Scenario: } & \begin{array}{l}\text { Air Quality - Excessive dust results in a personnel health hazard (see also U15017 and } \\ \text { Ui5070). }\end{array} \\ \text { Mitigation Feature: } & \begin{array}{l}\text { Monitor for out-of-tolerance or out-of-specified range conditions; when out of tolerance/range, } \\ \text { work ceases until mitigation is accomplished by respirator use and/or system/equipment repair } \\ \text { or adjustments. }\end{array}\end{array}$

Documentation: Procedures, JSA(s), safety manuals

Remarks:

Mitigation type:
Design Feature
Safety Device
Warning Device
Procedure
Training

\section{Mitigation Tracking}

\section{Mitigation Implementation Status:}

Comments:
Not Implemented

Implemented

Implemented (per T.Rotert, aug98.) wp

Appendix A

Page UI5060.2 


\section{DI: BAB000000-01717-0200-0004; Rev 02}

\section{Mitigation Feature}

Scenario Number: 415060 Mitigation Number: 3

Scenario: $\quad$ Air Quality - Excessive dust results in a personnel health hazard (see also U15017 and Ui5070).

Mitigation Feature: Respirator use as dictated by Industrial Hygienist.

Documentation: Procedures, JSA(s), safety manuals

Remarks:

Mitigation type:
Design Feature
Safety Device
Warning Device
Procedure
Training

\section{Mitigation Tracking}

\section{Mitigation Implementation Status:}

Comments:
Cot Implemented
implemented

Implemented (per T.Rotert, aug98.) wp

\section{Appendix A}

Page UI5060.3 


\section{DI: BAB000000-01717-0200-0004; Rev 02}

\section{Mitigation Feature}

Scenario Number:

U15060

Mitigation Number:

4

Scenario:

Air Quality - Excessive dust results in a personnel health hazard (see also U15017 and U(5070).

Mitigation Feature: Use water sprays or other dust suppression materials to wet down excavated walls and muck pile.

Documentation: Procedures, JSA(s), safety manuals

Remarks:

Mitigation type:
Design Feature
Safety Device
Warning Device
Procedure
Training

\section{Mitigation Tracking}

Mitigation

Implementation

Status:

Comments:
Not Implemented

Implemented

Appendix A

Page UI5060.4 


\section{DI: BAB000000-01717-0200-0004, Rev 02}

\begin{tabular}{|c|c|c|}
\hline \multicolumn{3}{|c|}{ Mitigation Feature } \\
\hline Scenario Number: & Mitigation Number: & 5 \\
\hline Scenario: & $\begin{array}{l}\text { Air Quality - Excessive dust results in a personnel health } \\
\text { U15070). }\end{array}$ & azard isee also UI5017 and \\
\hline Mitigation Feature: & Provide enhanced joint seal (i.e., rubber band plus metal & uct band) on ventilation ducts. \\
\hline Documentation: & Procedures, JSA(s), safety manuals & \\
\hline Remarks: & & \\
\hline Mitigation type: & $\begin{array}{l}\text { Design Feature } \\
\text { Safety Device } \\
\text { warning Device } \\
O_{\text {Procedure }} \\
\text { Training }\end{array}$ & \\
\hline
\end{tabular}

\section{Mitigation Tracking}

\section{Mitigation Implementation Status:}

Comments:

\section{Not Implemented}

Implemented 


\section{DI: BAB000000-01717-0200-0004; Rev 02}

\begin{tabular}{|c|c|c|}
\hline \multicolumn{3}{|c|}{ Mitigation Feature } \\
\hline Scenario Number: & Mitigation Number: & 6 \\
\hline Scenario: & $\begin{array}{l}\text { Air Quality - Excessive dust results in a personnel health } \\
\text { U15070). }\end{array}$ & lazard (see also U15017 and \\
\hline Mitigation Feature: & JSAs, procedures and/or training will address hazards ass & ociated with operational activities. \\
\hline Documentation: & Procedures, JSA $\{$ s\}, safety manuals & \\
\hline Remarks: & & \\
\hline Mitigation type: & $\begin{array}{l}\bigcirc \text { Design Feature } \\
\text { Safety Device } \\
\text { Warning Device } \\
\text { Procedure } \\
\text { Training }\end{array}$ & \\
\hline
\end{tabular}

\section{Mitigation Tracking}

\section{Mitigation Implementation Status:}

Comments:
Not Implemented

Implemented

Implemented (per T.Rotert, aug98.) wp

\section{Appendix $A$}

Page UI5060.6 


\section{Scenario Description and Analysis}

\section{Approved}

Scenario Number:

Revision Number:

Revision Date:

Scenario:

Cause, Failure, or Hazardous Event:

Risk Assignment Before Considering Mitigations:

\begin{tabular}{|l|}
\hline U15061 \\
\hline 02 \\
\hline $12 / 17 / 98$ \\
\hline
\end{tabular}
potential sources of noise. activities.

\author{
SSA: East-West Drift \\ Location: East-West Drift
}

Excessive noise due to vehicles, rock bolt drills, ventilation fans, drilling/blasting, or other

Normal drill \& blast/excavation activities, transport systems, and ground support installation

\begin{tabular}{|lll|}
\hline Frequency Rating: & Consequence Rating: & *Risk Before: \\
A - Frequent & $O_{\text {- Catastrophic }}$ & \\
B - Probable & II - Critical & High \\
C - Occasional & $O$ III - Marginal & \\
D-Remote & IV - Negligible & \\
E- Improbable & & \\
\hline
\end{tabular}

Risk Designation

\section{After}

Applying

Mitigations:

\begin{tabular}{|lll|}
\hline Frequency Rating: & Consequence Rating: & Risk After: \\
A - Frequent & $\bigcirc I-$ Catastrophic & \\
B - Probable & $\bigcirc \|$ - Critical & Extremely Low \\
C - Occasional & $\bigcirc I I-$ Marginal & \\
D- Remote & $O I V-$ Negligible & \\
E - Improbable & & \\
\hline
\end{tabular}

Related Scenarios:

Prevention

Mitigation

Documentation: 


\section{DI: BAB000000-01717-0200-0004, Rev 02}

\section{Mitigation Feature}

Scenario Number: U15061 Mitigation Number: 1

Scenario:

Excessive noise due to vehicles, rock bolt drills, ventilation fans, drilling/blasting, or other potential sources of noise.

Mitigation Feature: Implement the Hearing Conservation Program (PRO-SH-004) and Medical Surveillance Program (NAP-SH-007) to minimize exposure to excessive noise levels.

Documentation: Procedures, JSA(s), safety manuals

Remarks:

Mitigation type: $\bigcirc$ Design Feature

Safety Device

Warning Device

Procedure

Training

Mitigation Tracking

Mitigation Implementation Status:

Not Implemented

Implemented

Comments:

Appendix A

Page UI5061.1 


\section{DI: BAB000000-01717-0200-0004; Rev 02}

\section{Mitigation Feature}

Scenario Number: $415061 \quad$ Mitigation Number: 2

Scenario:

Excessive noise due to vehicles, rock bolt drills, ventilation fans, drilling/blasting, or other potential sources of noise.

Mitigation Feature: Periodically measure for out-of-tolerance or out-of-specified range conditions; when out of tolerance/range, provide ear protection for immediate mitigation.

Documentation: $\quad$ Procedures, JSA $\{$ s\}, safety manuals

Remarks: $\quad$ Rev.01, Mit \#6

Mitigation type: $\quad \mid \begin{aligned} & \bigcirc \text { Design Feature } \\ & \bigcirc \text { Safety Device } \\ & \bigcirc \text { Warning Device } \\ & \bigcirc \text { Procedure } \\ & \bigcirc \text { Training }\end{aligned}$

\section{Mitigation Tracking}

Mitigation Implementation Status:

Comments:
Not implemented

Implemented 


\section{DI: BAB000000-01717-0200-0004; Rev 02}

\section{Mitigation Feature}

Scenario Number: $415061 \quad$ Mitigation Number: 3

Scenario: $\quad$ Excessive noise due to vehicles, rock bolt drills, ventilation fans, drilling/blasting, or other potential sources of noise.

Mitigation Feature: Clear area prior to blasting.

Documentation: $\quad$ Procedures, JSA(s), safety manuals

Remarks:

Mitigation type:
Design Feature
Safety Device
Warning Device
Procedure
Training

\section{Mitigation Tracking}

Mitigation Implementation Status:

Comments:
Not Implemented

Implemented

Implemented (per T.Rotert, aug98.) wp

\section{Appendix A}

Page U15061.3 


\section{DI: BAB000000-01717-0200-0004, Rev 02}

\begin{tabular}{|c|c|c|}
\hline \multicolumn{3}{|c|}{ Mitigation Feature } \\
\hline Scenario Number: & Mitigation Number: & 4 \\
\hline Scenario: & $\begin{array}{l}\text { Excessive noise due to vehicles, rock bolt drills, ventilatio } \\
\text { potential sources of noise. }\end{array}$ & fans, drilling/blasting, or other \\
\hline Mitigation Feature: & Ventilation fans purchased/installed shall meet ACGIH sta & ndard for noise. \\
\hline Documentation: & Purchase specification, ACGIH standard. & \\
\hline Remarks: & & \\
\hline Mitigation type: & $\begin{array}{l}\text { Design Feature } \\
\text { Safety Device } \\
\text { Warning Device } \\
\text { Procedure } \\
\text { Training }\end{array}$ & \\
\hline
\end{tabular}

\section{Mitigation Tracking}

Mitigation Implementation

Status:

Comments:

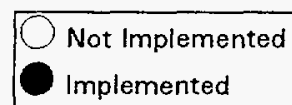

Implemented (per T.Rotert, aug98 Purchase specs require noise levels below ACGIH standard.) wp
Appendix A Page UI5061.4 


\section{DI: BAB000000-01717-0200-0004; Rev 02}

\section{Mitigation Feature}

Scenario Number:

U15061 Mitigation Number:

5

Scenario:

Excessive noise due to vehicles, rock bolt drills, ventilation fans, drilling/blasting, or other potential sources of noise.

Mitigation Feature: JSAs, procedures and/or training will address hazards associated with operational activities.

Documentation: $\quad$ Procedures, JSA(s), safety manuals

Remarks:

Remarks:

Mitigation type:

$$
\begin{aligned}
& \bigcirc \text { Design Feature } \\
& \text { Safety Device } \\
& \bigcirc \text { Warning Device } \\
& \text { Procedure } \\
& \text { Training }
\end{aligned}
$$

\section{Mitigation Tracking}

Mitigation Implementation Status:

Comments:

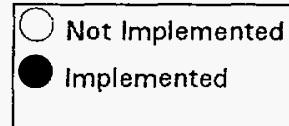

Implemented (per T.Rotert, aug98.) wp

Appendix A

Page U15061.5 


\section{Scenario Description and Analysis}

\section{Approved}

\section{Scenario Number: \\ Revision Number: \\ Revision Date:}

Scenario:

Cause, Failure, or Hazardous Event:

Risk Assignment
Before Considering
Mitigations:

Risk Designation

\section{After}

Applying

Mitigations:

\author{
SSA: East-West Drift \\ Location: East-West Drift
}

Worker injury due to fall from an elevated platform.

Personnel negligence, slippery surface, material spills, failure to adhere to safety procedures and rules.

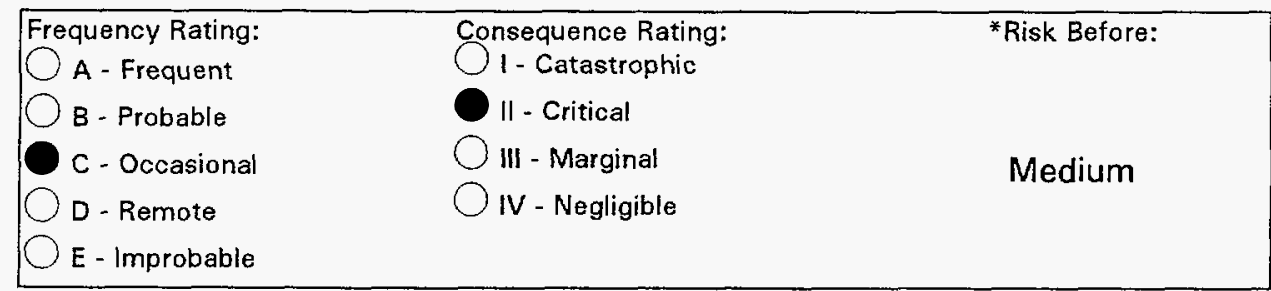

\begin{tabular}{|c|c|c|}
\hline $\begin{array}{l}\text { Frequency Rating: } \\
\text { OA - Frequent } \\
\text { O - Probable } \\
\text { O - Occasional } \\
\text { OD-Remote } \\
\text { E- Improbable }\end{array}$ & $\begin{array}{l}\text { Consequence Rating: } \\
\text { II - Catastrophic } \\
\text { II - Critical } \\
\text { III - Marginal } \\
\text { IV - Negligible }\end{array}$ & Risk After: \\
\hline
\end{tabular}

Related Scenarios:

U10108

Prevention

Mitigation

Safety manual; OSHA - 29 CFR 1926 Subparts $E_{i} M, X_{i}$ procedures and operators manuals; maintenance manuais; personnel training records.

Documentation:

\section{Appendix A \\ Page UI5062}




\section{DI: BAB000000-01717-0200-0004; Rev 02}

\section{Mitigation Feature}

Scenario Number: $u 15062$ Mitigation Number: 1

Scenario: $\quad$ Worker injury due to fall from an elevated platform.

Mitigation Feature: Handrail and toe plate around all landings, platforms and stairs per OSHA standards.

Documentation: OSHA - 29 CFR 1926 Subparts $E, M, X$; maintenance manuals.

Remarks:

Mitigation type:
Design Feature
Safety Device
Warning Device
Procedure
Training

\section{Mitigation Tracking}

Mitigation

Implementation

Status:
Not Implemented
Implemented

Comments:

Implemented (per T.Rotert, aug98.) wp

\section{Appendix A}

Page UI5062.1 


\section{DI: BAB000000-01717-0200-0004, Rev 02}

\section{Mitigation Feature}

Scenario Number:

Scenario:

Mitigation Feature:

Documentation:

Remarks:

Mitigation type:

UI5062 Mitigation Number:

2

Worker injury due to fall from an elevated platform.

Establish JSA procedures for elevated platform operations and maintenance.

Safety manual; OSHA - 29 CFR 1926 Subparts $E, M, X$; procedures and operators manuals; maintenance manuals; personnel training records.

Design Feature
Safety Device
Warning Device
Procedure
Training

\section{Mitigation Tracking}

Mitigation Implementation Status:

Comments:
Not Implemented

Implemented

Appendix A

Page UI5062.2 


\section{DI: BAB000000-01717-0200-0004, Rev 02}

\section{Mitigation Feature}

Scenario Number: U15062 Mitigation Number:

Scenario: $\quad$ Worker injury due to fall from an elevated platform

Mitigation Feature: Provide gate or chain across openings, where appropriate.

Documentation: Safety manual; OSHA - 29 CFR 1926 Subparts E, M, X; procedures and operators manuals: maintenance manuals; personnel training records.

Remarks:

Mitigation type: $\bigcirc$ Design Feature

Safety Device

Warning Device

Procedure

Training

\section{Mitigation Tracking}

Mitigation Implementation Status:

Comments:
Not Implemented

implemented

Implemented (per T.Rotert, aug98.) wp

\section{Appendix A}

Page UI5062.3 


\section{DI: BAB000000-01717-0200-0004, Rev 02}

\section{Mitigation Feature}

Scenario Number:

U15062 Mitigation Number:

4

Scenario: $\quad$ Worker injury due to fall from an elevated platform.

Mitigation Feature: Implement OSHA requirements for usage of safety belts during work in unprotected areas.

Documentation: $\quad$ Safety manual; OSHA - 29 CFR 1926 Subparts E, M, X; procedures and operators manuals; maintenance manuals; personnel training records.

Remarks:

Mitigation type:

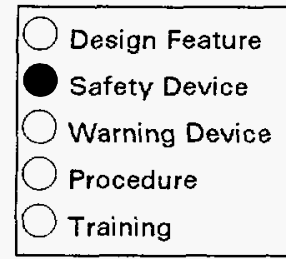

\section{Mitigation Tracking}

Mitigation

Implementation

Status:

Comments:
Not Implemented

Implemented

Implemented (per T.Rotert, aug98.) wp

Appendix A

Page U15062.4 


\section{DI: BAB000000-01717-0200-0004, Rev 02}

\section{Mitigation Feature}

Scenario Number:

U15062 Mitigation Number: 5

Scenario:

Worker injury due to fall from an elevated platform.

Mitigation Feature: Provide safety training using JSA procedures as a guide for work on elevated platforms.

Documentation: Safety manual; OSHA - 29 CFR 1926 Subparts $E, M, X$; procedures and operators manuals; maintenance manuals; personnel training records.

Remarks:

Mitigation type:
Design Feature
Safety Device
Warning Device
Procedure
Training

\section{Mitigation Tracking}

Mitigation Implementation Status:

Comments:
Not Implemented

implemented 


\section{Scenario Description and Analysis}

\section{Approved}

Scenario Number:

Revision Number:

Revision Date:

Scenario:

Cause, Failure, or Hazardous Event:

Risk Assignment Before Considering Mitigations:

\begin{tabular}{|l|}
\hline U15067 \\
\hline 02 \\
\hline $12 / 17 / 98$ \\
\hline
\end{tabular}
communications line, data line).

Subsurface vehicle (e.g., front-end loader) or machinery collision with utility cable, conduit or pipe le.g., ventilation, water supply, subsurface waste water, compressed air, electrical line,

Failure to install utilities clear of travel envelope; failure to provide protective barriers which protect utilities in the event of a collision; failure to properly prepare equipment for travel (e.g., set brakes) and/or restrain equipment so that it is not ejected from the vehicle in the event of a collision; loss of control over vehicle.

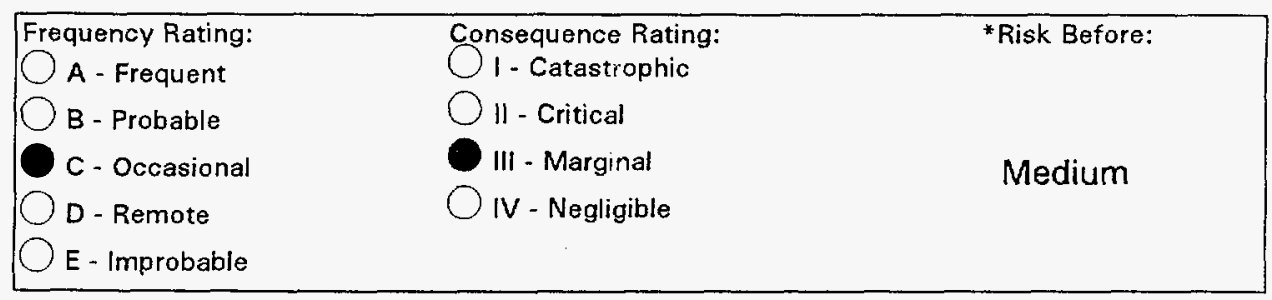

Risk Designation

After

Applying

Mitigations:

\begin{tabular}{|lll|}
\hline Frequency Rating: & Consequence Rating: & Risk After: \\
A - Frequent & OI-Catastrophic & \\
B - Probabie & II - Critical & Extremely Low \\
C - Occasional & III- Marginal & \\
D-Remote & IV - Negligible & \\
E- Improbable & & \\
\hline
\end{tabular}

Related Scenarios:

$\overline{\mathrm{U} 10051}$

Prevention
Mitigation
Documentation:

Title II Design Drawings; vehicle specifications; training manuals; operator manuals; safety manual; JSAs. 


\section{DI: BAB000000-01717-0200-0004; Rev 02}

\section{Mitigation Feature}

Scenario Number: $\quad$ U15067 Mitigation Number: 1

Scenario:

Subsurface vehicle (e.g., front-end loader) ar machinery collision with utility cable, conduit or pipe (e.g., ventilation, water supply, subsurface waste water, compressed air, electrical line, communications line, data line).

Mitigation Feature: Design drifts and alcoves large enough to provide adequate space for utilities, taking into account all of the equipment that will be used in these confined spaces.

Documentation:

Title II Design Drawings; vehicle specifications; training manuals; operator manuals; safety manual; JSAs.

Remarks:

Mitigation type:

Design Feature

Safety Device

Warning Device

Procedure

Training

\section{Mitigation Tracking}

\section{Mitigation Implementation Status:}

Comments:
Not Implemented

Implemented

Implemented (per T.Rotert, aug98.) wp

\section{Appendix A}

Page UI5067.1 


\section{DI: BAB000000-01717-0200-0004; Rev 02}

\section{Mitigation Feature}

Scenario Number: $\quad$ U15067 Mitigation Number: 2

Scenario:

Subsurface vehicle (e.g., front-end loader) or machinery collision with utility cable, conduit or pipe (e.g., ventilation, water supply, subsurface waste water, compressed air, electrical line, communications line, data line).

Mitigation Feature: Select equipment/materials/supplies that are compatible with the transportation envelope.

Documentation: Title II Design Drawings; vehicle specifications; training manuals; operator manuals; safety manual; JSAs.

Remarks:

Mitigation type:

Design Feature

Safety Device

Warning Device

Procedure

Training

\section{Mitigation Tracking}

Mitigation Implementation

Status:

Comments:
Not Implemented

Implemented

Implemented (per T.Rotert, aug98.) wp 


\section{DI: BAB000000-01717-0200-0004, Rev 02}

\begin{tabular}{|c|c|}
\hline \multicolumn{2}{|r|}{ Mitigation Feature } \\
\hline Scenario Number: & Mitigation Number: \\
\hline Scenario: & $\begin{array}{l}\text { Subsurface vehicle (e.g., front-end loader) or machinery collision with utility cable, conduit or } \\
\text { pipe (e.g., ventilation, water supply, subsurface waste water, compressed air, electrical line, } \\
\text { communications line, data line). }\end{array}$ \\
\hline Mitigation Feature: & $\begin{array}{l}\text { Develop operating procedures that prohibit equipment travel without having extendible } \\
\text { appendages retracted and/or properly restrained. }\end{array}$ \\
\hline Documentation: & $\begin{array}{l}\text { Title II Design Drawings; vehicle specifications; training manuals; operator manuals; safety } \\
\text { manual; JSAs. }\end{array}$ \\
\hline Remarks: & \\
\hline Mitigation type: & $\begin{array}{l}\text { Design Feature } \\
\text { Safety Device } \\
\text { warning Device } \\
\text { Procedure } \\
\bigcirc \text { Training }\end{array}$ \\
\hline
\end{tabular}

\section{Mitigation Tracking}

Mitigation

Implementation

Status:

Comments:
ONot Implemented

Implemented 


\section{DI: BAB000000-01717-0200-0004, Rev 02}

\section{Mitigation Feature}

Scenario Number: $\quad$ U15067 Mitigation Number: 4

Scenario:

Subsurface vehicle (e.g., front-end loader) or machinery collision with utility cable, conduit or pipe (e.g., ventilation, water supply, subsurface waste water, compressed air, electrical line, communications line, data line).

Mitigation Feature: Design ground fault protection into electrical systems where appropriate.

Documentation: Title II Design Drawings; vehicle specifications; training manuals; operator manuals; safety manual; JSAs.

Remarks:

Mitigation type:

Design Feature

Safety Device

Warning Device

Procedure

Training

\section{Mitigation Tracking}

Mitigation

Implementation

Status:

Comments:
Not Implemented

Implemented

Implemented (per T.Rotert, aug98.) wp

Appendix A

Page UI5067.4 


\section{DI: BAB000000-01717-0200-0004, Rev 02}

\section{Mitigation Feature}

Scenario Number:

U15067

Mitigation Number:

5

Scenario:

Subsurface vehicle (e.g., front-end loader) or machinery collision with utility cable, conduit or pipe (e.g., ventilation, water supply, subsurface waste water, compressed air, electrical line, communications line, data line).

Mitigation Feature: Design adequate clearance for the transportation envelope.

Documentation: Title II Design Drawings; vehicle specifications; training manuals; operator manuals; safety manual; JSAs.

Remarks:

Mitigation type:

Design Feature

Safety Device

Warning Device

Procedure

Training

\section{Mitigation Tracking}

Mitigation

Implementation

Status:

Not Implemented

Implemented

Comments:

Implemented (per T.Rotert, aug98.) wp

Appendix A

Page UI5067.5 


\section{DI: BAB000000-01717-0200-0004; Rev 02}

\section{Mitigation Feature}

Scenario Number: $415067 \quad$ Mitigation Number: 6

Scenario: $\quad$ Subsurface vehicle (e.g., front-end loader) or machinery collision with utility cable, conduit or pipe (e.g., ventilation, water supply, subsurface waste water, compressed air, electrical line, communications line, data line).

Mitigation Feature: Provide safety training for vehicle operators and personnel working around vehicles.

Documentation:

Remarks:

Mitigation type:
Design Feature
Safety Device
Warning Device
Procedure
Training

\section{Mitigation Tracking}

Mitigation

Implementation

Status:

Comments:
Not Implemented

Oimplemented

\section{Appendix A}

Page UI5067.6 


\section{Scenario Description and Analysis}

\section{Approved}

Scenario Number:

Revision Number:

Revision Date:

Scenario:

Cause, Failure, or Hazardous Event:

Risk Assignment Before Considering Mitigations:

Risk Designation

After

Applying

Mitigations:

Related Scenarios:

Prevention

Mitigation

Documentation:

\begin{tabular}{|l|}
\hline 015069 \\
\hline 02 \\
\hline $12 / 17 / 98$ \\
\hline
\end{tabular}

SSA: East-West Drift
Location: East-West Drift

General slips and trips resulting in severe personnel injury (see also UI5047).

Loss of off-site power; failure of emergency backup lighting; material spills; human error; lack of adequate illumination; debris (e.g., oil, water, tools) on surfaces; slippery surfaces; lack of non-skid surfaces; failure to adhere to safety procedures and rules.

\begin{tabular}{|lll|}
\hline Frequency Rating: & Consequence Rating: & *Risk Before: \\
A - Frequent & I-Catastrophic & \\
B - Probable & II - Critical & High \\
C - Occasional & III - Marginal & \\
D- Remote & $O I V$ - Negligible & \\
E-Improbable & & \\
\hline
\end{tabular}

\begin{tabular}{|lll|}
\hline Frequency Rating: & Consequence Rating: & Risk After: \\
A - Frequent & I- Catastrophic & \\
B - Probable & II - Critical & Low \\
C - Occasional & OIII- Marginal & \\
D - Remote & IV - Negligible & \\
E - improbable & & \\
\hline
\end{tabular}

UI5047 (minor slips and trips)

Inspection, maintenance and cleanup records; design drawings; maintenance manuals; safety manuals

\section{Appendix A \\ Page UI5069}


DI: BAB000000-01717-0200-0004, Rev 02

\begin{tabular}{|c|c|}
\hline & Mitigation Feature \\
\hline Scenario Number: & Mitigation Number: \\
\hline Scenario: & General slips and trips resulting in severe personnel injury (see also U15047). \\
\hline Mitigation Feature: & $\begin{array}{l}\text { Provide adequate and proper illumination in the ECRB for the expected population and work } \\
\text { activities. }\end{array}$ \\
\hline Documentation: & Inspection, maintenance and cleanup records; maintenance manuals; safety manuals \\
\hline Remarks: & \\
\hline Mitigation type: & $\begin{array}{l}\text { Design Feature } \\
\text { Safety Device } \\
\bigcirc \text { Warning Device } \\
\bigcirc \text { Procedure } \\
\bigcirc \text { Training }\end{array}$ \\
\hline
\end{tabular}

\section{Mitigation Tracking}

Mitigation

Implementation

Status:

Comments:
Not Implemented

Implemented

\section{Appendix A}

Page UI5069.1 


\section{DI: BAB000000-01717-0200-0004; Rev 02}

\section{Mitigation Feature}

Scenario Number: $\quad$ U15069 Mitigation Number: 2

Scenario: $\quad$ General slips and trips resulting in severe personnel injury (see also U15047).

Mitigation Feature: Provide emergency lighting for use during a power failure or lighting system failure.

Documentation: Inspection, maintenance and cleanup records; design drawings; maintenance manuals; safety manuals

Remarks:

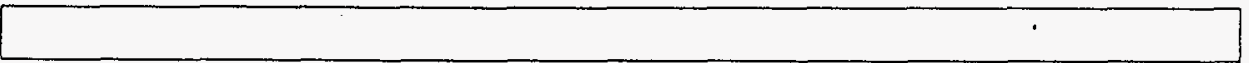

Mitigation type:

Design Feature

Safety Device

Warning Device

Procedure

Training

Mitigation Tracking

Mitigation

Implementation

Status:

Not Implemented

Omplemented

Comments:

\section{Appendix A}

Page UI5069.2 
DI: BAB000000-01717-0200-0004, Rev 02

\section{Mitigation Feature}

Scenario Number: U15069 Mitigation Number: 3

Scenario: $\quad$ General slips and trips resulting in severe personnel injury (see also UI5047).

Mitigation Feature: Use personal lighting (l.e., cap lamps) in unlighted or poorly illuminated areas.

Documentation: Inspection, maintenance and cleanup records; maintenance manuals; safety manuals

Remarks:

Mitigation type: $\bigcirc$ Design Feature

Safety Device

Warning Device

Procedure

Training

Mitigation Tracking

Mitigation

Implementation

Status:

Not Implemented

implemented

Comments:

Appendix A

Page UI5069.3 


\section{DI: BAB000000-01717-0200-0004, Rev 02}

\section{Mitigation Feature}

Scenario Number: U15069 Mitigation Number: 4

Scenario:

General slips and trips resulting in severe personnel injury (see also U15047).

Mitigation Feature:

Documentation:

Establish inspection, maintenance and good housekeeping rules.

inspection, maintenance and cleanup records; design drawings; maintenance manuals; safety manuals

Remarks:

Mitigation type:

Design Feature

Safety Device

Warning Device

Procedure

Training

\section{Mitigation Tracking}

Mitigation Implementation Status:

Implemented

Comments:

Appendix A

Page UI5069.4 
DI: BAB000000-01717-0200-0004; Rev 02

\section{Mitigation Feature}

Scenario Number:

U15069

Mitigation Number:

5

Scenario:

General slips and trips resulting in severe personnel injury (see also U15047).

Mitigation Feature: Provide first-aid kits on the TBM and at the entrance to the ECRB.

Documentation: Inspection, maintenance and cleanup records; maintenance manuals; safety manuals

Remarks:

Mitigation type:

Design Feature

Safety Device

Warning Device

Procedure

Training

\section{Mitigation Tracking}

Mitigation

Implementation

Status:

Not Implemented

Implemented

Comments:

Implemented (per T.Rotert, aug98.) wp

\section{Appendix A \\ Page UI5069.5}




\section{DI: BAB000000-01717-0200-0004; Rev 02}

\begin{tabular}{|c|c|c|}
\hline \multicolumn{3}{|c|}{ Mitigation Feature } \\
\hline Scenario Number: & Mitigation Number: & 6 \\
\hline Scenario: & General slips and trips resulting in severe personnel injury & (see aiso U15047). \\
\hline Mitigation Feature: & $\begin{array}{l}\text { Provide safety training and caution all personnel regarding } \\
\text { tunnel flooring. }\end{array}$ & uneven surfaces on platfarms and \\
\hline Documentation: & $\begin{array}{l}\text { Inspection, maintenance and cleanup records; design dra } \\
\text { manuals }\end{array}$ & vings; maintenance manuals; safety \\
\hline Remarks: & Rev.01, Mit \#7 & \\
\hline Mitigation type: & $\begin{array}{l}\text { Design Feature } \\
\text { Safety Device } \\
\text { Warning Device } \\
\text { Procedure } \\
\text { Training }\end{array}$ & \\
\hline
\end{tabular}

\section{Mitigation Tracking}

Mitigation Implementation

Status:

Comments:
Not Implemented

Implemented

Implemented (per T.Rotert, aug98.) wp

\section{Appendix A}

Page UI5069.6 


\section{Scenario Description and Analysis}

\section{Approved}

Scenario Number:

Revision Number:

Revision Date:

Scenario:

Cause, Failure, or Hazardous Event:

Risk Assignment Before Considering Mitigations:

\section{After}

Applying

Mitigations:

Related Scenarios:

Prevention

Mitigation

Documentation:

\begin{tabular}{|l|}
\hline U15070 \\
\hline 02 \\
\hline $12 / 17 / 98$ \\
\hline
\end{tabular}

SSA: East-West Drift

Location: East-West Drift

Air Quality - Excessive diesel soot and/or smoke particulate results in a personnel health hazard (see also UI5042).

Diesel vehicle exhaust system failure or out-of-tolerance condition; ventilation system failure or out-of-tolerance condition; failure to adhere to proper procedures (e.g., use wrong fuel or oil in vehicles)

\begin{tabular}{|llc|}
\hline Frequency Rating: & Consequence Rating: & *Risk Before: \\
A - Frequent & II-Catastrophic & \\
B - Probable & II - Critical & Medium \\
C - Occasional & III - Marginal & \\
D - Remote & IV - Negligible & \\
E - Improbable & & \\
\hline
\end{tabular}

\begin{tabular}{|lll|}
\hline Frequency Rating: & Consequence Rating: & Risk After: \\
A - Frequent & $O_{1-\text { Catastrophic }}$ & \\
B - Probable & $O_{I}$ - Critical & Extremely Low \\
C - Occasional & III - Marginal & \\
D - Remote & IV - Negligible & \\
E - Improbable & & \\
\hline
\end{tabular}

U15042

Vehicle operating and maintenance procedures, manuals and records; OSHA workplace exposure limits; JSA(s); Title II design drawings; training records; safety manual

Appendix A

Page UI5070 
DI: BAB000000-01717-0200-0004, Rev 02

\begin{tabular}{|c|c|}
\hline \multicolumn{2}{|r|}{ Mitigation Feature } \\
\hline Scenario Number: & Mitigation Number: \\
\hline Scenario: & $\begin{array}{l}\text { Air Quality - Excessive diesel soot and/or smoke particulate results in a personnel health } \\
\text { hazard (see also UI5042). }\end{array}$ \\
\hline Mitigation Feature: & JSAs, procedures and/or training will address hazards associated with operational activities. \\
\hline Documentation: & $\begin{array}{l}\text { Vehicle operating and maintenance procedures, manuals and records; OSHA workplace } \\
\text { exposure limits; MSHA } 30 \text { CFR Part } 7 \text {; JSA(s); training records; safety manual }\end{array}$ \\
\hline Remarks: & \\
\hline Mitigation type: & $\begin{array}{l}\bigcirc \text { Design Feature } \\
\text { Safety Device } \\
\text { Warning Device } \\
\text { Procedure } \\
\text { Training }\end{array}$ \\
\hline
\end{tabular}

\section{Mitigation Tracking}

\section{Mitigation Implementation Status:}

Comments:
Not Implemented

Implemented

\section{Appendix A}

Page UI5070.1 


\section{DI: BAB000000-01717-0200-0004, Rev 02}

\section{Mitigation Feature}

Scenario Number:

U15070 Mitigation Number:

Scenario:

Air Quality - Excessive diesel soot and/or smoke particulate results in a personnel health hazard (see also U15042).

Mitigation Feature:

Ventilation System removes exhaust smoke and suspended particulates. Also provides fresh air up wind of the diesel equipment.

Documentation:

Vehicle operating and maintenance procedures, manuals and records; OSHA workplace exposure limits; JSA(s); Title II design drawings; training records; safety manual

Remarks:

Mitigation type:

Design Feature

Safety Device

Warning Device

Procedure

Training

\section{Mitigation Tracking}

Mitigation Implementation Status: Not Implemented

Implemented

Comments:

Appendix A

Page UI5070.2 


\section{DI: BAB000000-01717-0200-0004, Rev 02}

\section{Mitigation Feature}

Scenario Number: $\quad$ U15070 Mitigation Number: 3

\section{Scenario: \\ Air Quality - Excessive diesel soot and/ar smoke particulate results in a personnel health} hazard isee also U15042\}.

Mitigation Feature: Establish vehicle inspection \& maintenance schedule.

Documentation: Vehicle operating and maintenance procedures, manuals and records; OSHA workplace exposure limits; JSA(s); Title II decin drawings; training records; safety manual

Remarks:

Rev.01, Mit \#6

Mitigation type:
Design Feature
Safety Device
Warning Device
Procedure
Training

\section{Mitigation Tracking}

\section{Mitigation Implementation Status:}

Comments:
Not Impiemented

Implemented

Implemented (per T.Rotert, aug98.) wp

\section{Appendix A \\ Page UI5070.3}




\section{DI: BAB000000-01717-0200-0004; Rev 02}

\section{Mitigation Feature}

Scenario Number:

U15070

Mitigation Number:

4

Scenario:

Air Quality - Excessive diesel soot and/or smoke particulate results in a personnel health hazard (see also U15042).

Mitigation Feature: Minimize idling of diesel engines.

Documentation: Vehicle operating and maintenance procedures, manuals and records; OSHA workplace exposure limits; JSA(s); Title II design drawings; training records; safety manual

Remarks:

Mitigation type:

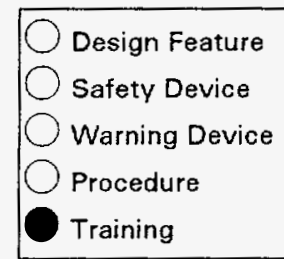

\section{Mitigation Tracking}

Mitigation Implementation Status: Not Implemented Implemented

Comments: implemented (per T.Rotert, aug98.) wp

Appendix A

Page UI5070.4 


\section{DI: BAB000000-01717-0200-0004, Rev 02}

\section{Mitigation Feature}

Scenario Number: 415070 Mitigation Number: 5

Scenario:

Air Quality - Excessive diesel soot and/or smoke particulate results in a personnel health hazard (see also UI5042).

Mitigation Feature: ${ }_{\text {Install scrubbers (catalytic converters) on subsurface diesel vehicles. }}$

Documentation: Vehicle operating and maintenance procedures, manuals and records; OSHA workplace exposure limits; JSA(s); Title II design drawings; training records; safety manual

Remarks:

Mitigation type: $\bigcirc$ Design Feature

Safety Device

Warning Device

Procedure

Training

\section{Mitigation Tracking}

Mitigation

Implementation

Status:

Comments:
Not Implemented

Implemented

Implemented (per T.Rotert, aug98.) wp

Appendix A

Page UI5070.5 


\section{DI: BAB000000-01717-0200-0004, Rev 02}

\section{Scenario Description and Analysis}

\section{Approved}

Scenario Number:

Revision Number:

Revision Date:

Scenario:

Cause, Failure, or Hazardous Event:

\section{Risk Assignment Before Considering Mitigations:}

\begin{tabular}{|l|}
\hline U15072 \\
\hline 02 \\
\hline $12 / 17 / 98$ \\
\hline
\end{tabular}

SSA: East-West Drift

Location: East-West Drift

Ventilation fan structural failure.

Equipment/component failure of bearing, blades, impeller assembly, nose cone or guide vanes.

\begin{tabular}{|lll|}
\hline Frequency Rating: & Consequence Rating: & *Risk Before: \\
A - Frequent & $\bigcirc$ I- Catastrophic & \\
B - Probable & II - Critical & Medium \\
C - Occasional & $\bigcirc I I$ - Marginal & \\
D- Remote & $\bigcirc$ IV - Negligible & \\
E- Improbable & & \\
\hline
\end{tabular}

\section{Risk Designation}

After

Applying

Mitigations:

\section{Frequency Rating: \\ A - Frequent \\ B - Probable \\ C - Occasional \\ OD - Remote \\ E - Improbable}

Consequence Rating:

Risk After:

O1 - Catastrophic

II - Critical

O III - Marginal

OIV - Negligible

Related Scenarios:

Ul0012, Ul0101, U10102, U10103

Prevention

Mitigation

Documentation:

ESF Subsurface Vaneaxial Ventilation Fans Procurement Specification and ESF Ventilation Duct and Fittings Procurement Specification. Ventilation fan service manual, operational procedures, safety manuals, maintenance manuals, training manuals.

\section{Appendix A}

Page UI5072 


\section{DI: BAB000000-01717-0200-0004, Rev 02}

\section{Mitigation Feature}

Scenario Number: U15072 Mitigation Number: 1

Scenario: $\quad$ Ventilation fan structural failure.

Mitigation Feature: Ventilation fan components are of high quality materials with proven performance record.

Documentation: ESF Subsurface Vaneaxial Ventilation Fans Procurement Specification and ESF Ventilation Duct and Fittings Procurement Specification. Ventilation fan service manual, operational procedures, safety manuals, maintenance manuals, training manuals.

Remarks:

,

Mitigation type:

Design Feature

Safety Device

Warning Device

Procedure

Training

\section{Mitigation Tracking}

Mitigation

Implementation

Status:

Not Implemented

Implemented

Comments:

Implemented (per T.Rotert, aug98.) wp

\section{Appendix A \\ Page UI5072.1}


DI: BABO00000-01717-0200-0004, Rev 02

\section{Mitigation Feature}

$\begin{array}{ll}\text { Scenario Number: } & \text { Ul5072 } \\ \text { Scenario: } & \text { Mentilation fan structural failure. } \\ \text { Mitigation Feature: } & \begin{array}{l}\text { The fan is inside a metal housing and connected to ventilation ducts. Provides missile } \\ \text { protection. }\end{array} \\ \text { Documentation: } & \begin{array}{l}\text { ESF Subsurface Vaneaxial Ventilation Fans Procurement Specification and ESF Ventilation } \\ \text { Duct and Fittings Procurement Specification. Ventilation fan service manual, operational } \\ \text { procedures, safety manuals, maintenance manuals, training manuals. }\end{array} \\ \text { Remarks: } & \begin{array}{l}\text { Design Feature } \\ \text { Mitigation type: }\end{array} \\ \text { Safety Device } \\ \text { Warning Device } \\ \text { Procedure } \\ \text { Training }\end{array}$

\section{Mitigation Tracking}

Mitigation Implementation Status: implemented

Comments: Implemented (per T.Rotert, aug98.) wp

\section{Appendix A} Page UI5072.2 


\section{DI: BAB000000-01717-0200-0004; Rev 02}

\section{Mitigation Feature}

Scenario Number:

U15072 Mitigation Number: 3

Scenario:

Ventilation fan structural failure.

Mitigation Feature: Provide for vibration and bearings temperature inspections.

Documentation: ESF Subsurface Vaneaxial Ventilation Fans Procurement Specification and ESF Ventilation Duct and Fittings Procurement Specification. Ventilation fan service manual, operational procedures, safety manuals, maintenance manuals, training manuals.

Remarks:

Mitigation type:

Design Feature

Safety Device

Warning Device

Procedure

Training

\section{Mitigation Tracking}

\section{Mitigation \\ Implementation \\ Status:}

Comments:

\section{Appendix A}

Page UI5072.3 
DI: BAB000000-01717-0200-0004; Rev 02

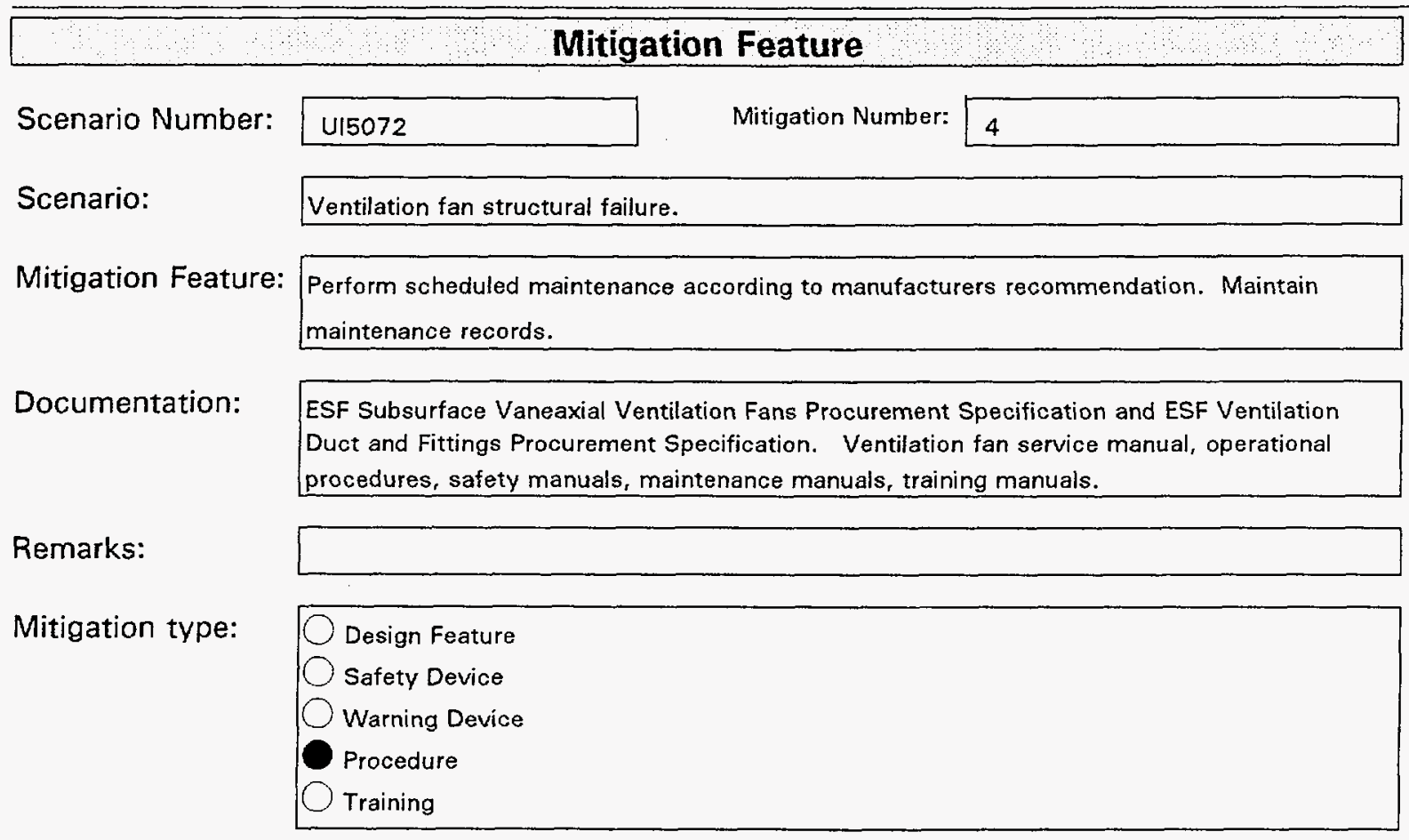

\section{Mitigation Tracking}

Mitigation Implementation

Status:

Comments:
Not Implemented
Implemented

Implemented (per T.Rotert, aug98.) wp 


\section{DI: BAB000000-01717-0200-0004, Rev 02}

\section{Scenario Description and Analysis}

\section{Approved}

Scenario Number:

Revision Number:

Revision Date:

Scenario:

Cause, Failure, or Hazardous Event:

Risk Assignment Before Considering Mitigations:

Risk Designation After Applying Mitigations:

\begin{tabular}{|l|}
\hline$U 15073$ \\
\hline 02 \\
\hline $12 / 17 / 98$ \\
\hline
\end{tabular}

Personnel injury/equipment damage due to muck falling off the conveyor system.

Conveyor component failure (e.g., conveyor belt, support rollers, etc.). Failure to follow safety procedures or operating/maintenance procedures $/ e . g .$, walking/crawling under a running conveyor belt, attempting maintenance when the system is not shut off

\begin{tabular}{|lll|}
\hline Frequency Rating: & Consequence Rating: & *Risk Before: \\
A - Frequent & I- Catastrophic & \\
B - Probable & II - Critical & Medium \\
C- Occasional & III - Marginal & \\
D - Remote & OIV - Negligible & \\
E- Improbable & & \\
\hline
\end{tabular}

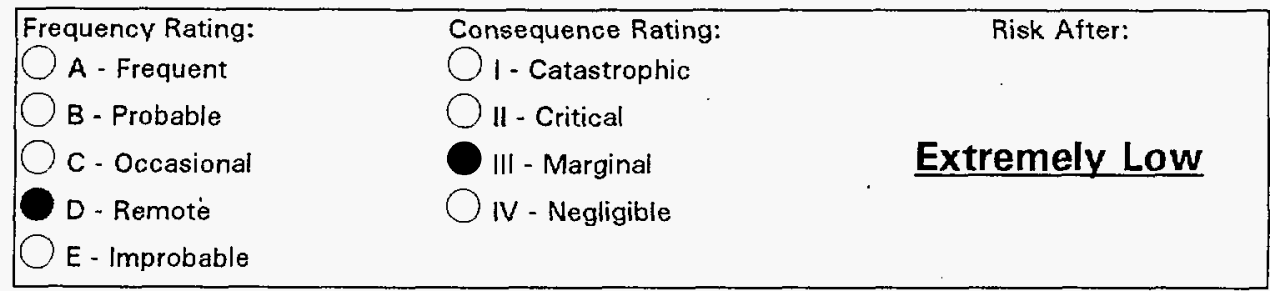

Related Scenarios:

\section{U10023}

Prevention

System specifications and design drawings. Maintenance and safety manuals.
Mitigation

Documentation:

\section{Appendix A \\ Page UI5073}




\section{DI: BAB000000-01717-0200-0004, Rev 02}

\section{Mitigation Feature}

Scenario Number: $\quad$ U15073 Mitigation Number: 1

Scenario:

Personnel injury/equipment damage due to muck falling off the conveyor system.

Mitigation Feature: Provide guards at transfer points to prevent muck from falling off the conveyor belt or between the conveyor belt and support structure.

Documentation:

Remarks:

Mitigation type:

Design Feature

Safety Device

Warning Device

Procedure

Training

\section{Mitigation Tracking}

Mitigation Implementation

Status:

Comments:
Not Implemented

Implemented

Implemented (per T.Rotert, aug98.) wp 


\section{DI: BAB000000-01717-0200-0004, Rev 02}

\section{Mitigation Feature}

Scenario Number:

U15073 Mitigation Number:

2

Scenario:

Personnel injury/equipment damage due to muck falling off the conveyor system.

Mitigation Feature:

Provide lockout/tagouts. Prevents system start up while in maintenance or inoperable status

Documentation:

Remarks:

Mitigation type:
Design Feature
Safety Device
Warning Device
Procedure
Training

\section{Mitigation Tracking}

Mitigation

Implementation

Status:

Comments:

\section{Not Implemented}

Implemented

Implemented (per T.Rotert, aug98.) wp

\section{Appendix A}

Page UI5073.2 
DI: BAB000000-01717-0200-0004, Rev 02

\section{Mitigation Feature}

Scenario Number: $\quad$ U15073 Mitigation Number: 3

Scenario: $\quad$ Personnel injury/equipment damage due to muck falling off the conveyor system.

Mitigation Feature: Personnel must keep hands and arms inside the train car while transiting the tunnel in order to avoid the conveyor structure.

Documentation:

Remarks:

Mitigation type:
Design Feature
Safety Device
Warning Device
Procedure
Training

\section{Mitigation Tracking}

Mitigation

Implementation

Status:

Comments:
Not Implemented

Implemented

Implemented (per T.Rotert, aug98_ Mancar openings are towards the utilities.) wp

\section{Appendix A \\ Page UI5073.3}




\section{DI: BAB000000-01717-0200-0004, Rev 02}

\begin{tabular}{|c|c|c|}
\hline \multicolumn{3}{|c|}{ Mitigation Feature } \\
\hline Scenario Number: & Mitigation Number: & 4 \\
\hline Scenario: & Personnel injury/equipment damage due to muck falling o & $f$ the conveyor system. \\
\hline Mitigation Feature: & $\begin{array}{l}\text { Require personnel to wear personal protective equipment } \\
\text { toed shoes) }\end{array}$ & e.g., hard hats, eye protection, hard \\
\hline Documentation: & & \\
\hline Remarks: & & \\
\hline Mitigation type: & $\begin{array}{l}\text { Design Feature } \\
\text { Safety Device } \\
\text { Warning Device } \\
\text { Procedure } \\
\text { Training }\end{array}$ & \\
\hline
\end{tabular}

\section{Mitigation Tracking}

Mitigation Implementation Status:

Comments:

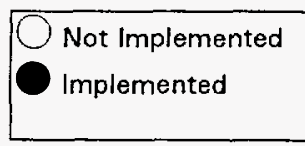

Implemented (per T.Rotert, aug98.) wp

\section{Appendix A}

Page UI5073.4 
DI: BAB000000-01717-0200-0004, Rev 02

\section{Mitigation Feature}

Scenario Number: $\quad$ U15073 Mitigation Number: 5

Scenario:

Personnel injury/equipment damage due to muck falling off the conveyor system.

Mitigation Feature: Establish inspection and maintenance procedures (e.g., alignment connections, guide wear) and schedule. Maintain inspection and maintenance records.

Documentation:

Remarks:

Mitigation type:
Design Feature
Safety Device
Warning Device
Procedure
$\bigcirc$ Training

\section{Mitigation Tracking}

\section{Mitigation Implementation Status:}

Comments:

\section{Not Implemented}

Implemented

To be implemented with the Maximo system. [Bob Law 05/19/99]

\section{Appendix A \\ Page UI5073.5}


DI: BAB000000-01717-0200-0004; Rev 02

\begin{tabular}{|c|c|c|}
\hline \multicolumn{3}{|c|}{ Mitigation Feature } \\
\hline Scenario Number: & Mitigation Number: & 6 \\
\hline Scenario: & Personnel injury/equipment damage due to muck falling o & f the conveyor system. \\
\hline Mitigation Feature: & $\begin{array}{l}\text { Provide safety training (e.g., rules and regulations pertain } \\
\text { exclusion area, etc.). }\end{array}$ & ng to personnel safety, personnel \\
\hline Documentation: & & \\
\hline Remarks: & & \\
\hline Mitigation type: & $\begin{array}{l}\text { Design Feature } \\
\bigcirc \text { Safety Device } \\
\bigcirc \text { Warning Device } \\
\bigcirc \text { Procedure } \\
\text { Training }\end{array}$ & \\
\hline
\end{tabular}

\section{Mitigation Tracking}

Mitigation Implementation Status:

Comments:
Not Implemented
Implemented

Implemented (per T.Rotert, aug98 Covered in GUT training.) wp

Appendix A

Page UI5073.6 


\section{DI: BAB000000-01717-0200-0004, Rev 02}

\section{Scenario Description and Analysis}

\section{Approved}

\section{Scenario Number: \\ Revision Number: \\ Revision Date:}

Scenario:

Cause, Failure, or Hazardous Event:

\section{Risk Assignment Before Considering Mitigations:}

\begin{tabular}{|l|}
\hline$U 15075$ \\
\hline 02 \\
\hline $12 / 17 / 98$ \\
\hline
\end{tabular}

SSA: East-West Drift

Location: East-West Drift
Personnel injury resulting from misuse of rail cars for personnel transit.

Action(s) by an individual cause exposure of the body (e.g., head, arms) beyond the upper and side limits of the rail transit window.

\begin{tabular}{|lll|}
\hline Frequency Rating: & Consequence Rating: & ${ }^{*}$ Risk Before: \\
A - Frequent & I-Catastrophic & \\
B - Probable & II - Critical & High \\
C - Occasional & $\bigcirc I I$ - Marginal & \\
D - Remote & IV - Negligible & \\
E- Improbable & & \\
\hline
\end{tabular}

\section{Risk Designation}

After

Applying

Mitigations:

Related Scenarios:

Prevention

Mitigation

Documentation:

Frequency Rating:
A - Frequent
B - Probable
C - Occasional
D - Remote
E - Improbable

29 CFR 1926.800. Vehicle and equipment specifications. Operation and maintenance manuals. Training and safety manuals.
Consequence Rating:
I - Catastrophic
II - Critical
OIII - Marginal
OIV - Negligible

Risk After:

Low

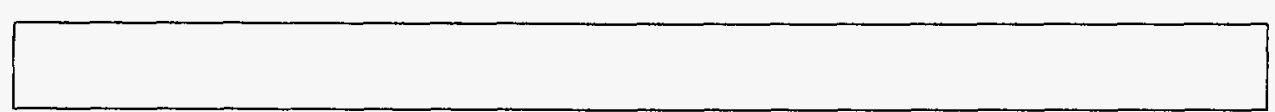

manuals. $T$ 


\section{DI: BAB000000-01717-0200-0004, Rev 02}

\section{Mitigation Feature}

$\begin{array}{ll}\text { Scenario Number: } & \text { Mit5075 } \\ \text { Scenario: } & \text { Personnel injury resulting from misuse of rail cars for personnel transit. } \\ \text { Mitigation Feature: } & \begin{array}{l}\text { Personnel must ride within authorized personnel compartments. Prohibit personnel from riding } \\ \text { on or outside (hanging on) vehicles, or material cars. Train operators will not move the train } \\ \text { until all personnel are properly seated. }\end{array}\end{array}$

Documentation:

Remarks:

\begin{tabular}{l|l} 
Mitigation type: & \begin{tabular}{|l}
$\bigcirc$ Design Feature \\
Safety Device \\
$\bigcirc$ Warning Device \\
Procedure \\
Training
\end{tabular} \\
\hline
\end{tabular}

\section{Mitigation Tracking}

Mitigation Implementation Status:

Comments:
Not Implemented

Implemented

Appendix A

Page U15075.1 


\section{DI: BAB000000-01717-0200-0004; Rev 02}

\begin{tabular}{|c|c|c|}
\hline \multicolumn{3}{|c|}{ Mitigation Feature } \\
\hline Scenario Number: & Mitigation Number: & 2 \\
\hline Scenario: & Personnel injury resulting from misuse of rail cars for pers & onnel transit. \\
\hline Mitigation Feature: & $\begin{array}{l}\text { Prohibit mixed loads on rail cars. Personnel and materials } \\
\text { Prohibit personnel from riding on, or holding materials and }\end{array}$ & $\begin{array}{l}\text { must be transported separately. } \\
\text { or equipment in place. }\end{array}$ \\
\hline Documentation: & & \\
\hline Remarks: & & \\
\hline Mitigation type: & $\begin{array}{l}\text { Design Feature } \\
\text { Safety Device } \\
\text { Warning Device } \\
\text { Procedure } \\
\text { Training }\end{array}$ & \\
\hline
\end{tabular}

\section{Mitigation Tracking}

Mitigation Implementation Status:

Comments:

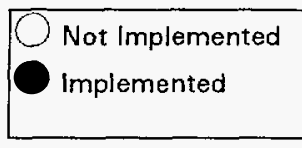

Implemented (per T.Rotert, aug98.) wp

\section{Appendix A \\ Page UI5075.2}




\section{DI: BAB000000-01717-0200-0004; Rev 02}

\begin{tabular}{|c|c|}
\hline \multicolumn{2}{|r|}{ Mitigation Feature } \\
\hline Scenario Number: & Mitigation Number: \\
\hline Scenario: & Personnel injury resulting from misuse of rail cars for personnel transit. \\
\hline Mitigation Feature: & $\begin{array}{l}\text { Design/modify personnel cars with high sides to prevent head, hands and arms from extending } \\
\text { beyond the train. }\end{array}$ \\
\hline Documentation: & $\begin{array}{l}29 \text { CFR } 1926.800 \text {. Vehicle and equipment specifications. Operation and maintenance } \\
\text { manuals. Training and safety manuals. }\end{array}$ \\
\hline Remarks: & \\
\hline Mitigation type: & $\begin{array}{l}\text { Design Feature } \\
\text { Safety Device } \\
\bigcirc \text { Warning Device } \\
\bigcirc \text { Procedure } \\
\bigcirc \text { Training }\end{array}$ \\
\hline
\end{tabular}

\section{Mitigation Tracking}

Mitigation Implementation Status:

Comments:
Not Implemented

Implemented

Implemented (per T.Rotert, aug98.) wp

Appendix A

Page UI5075.3 


\section{DI: BAB000000-01717-0200-0004; Rev 02}

\section{Mitigation Feature}

Scenario Number:

U15075 Mitigation Number: 4

Scenario:

Personnel injury resulting from misuse of rail cars for personnel transit.

Mitigation Feature: Operators will not move the train until all personnel are properly seated.

Documentation:

29 CFR 1926.800. Vehicle and equipment specifications. Operation and maintenance manuals. Training and safety manuals.

Remarks:

Mitigation type:
Design Feature
Safety Device
Warning Device
Procedure
Training

\section{Mitigation Tracking}

Mitigation Implementation

Status:

Comments:
Not Implemented

Implemented 


\section{Scenario Description and Analysis}

\section{Approved}

Scenario Number:

Revision Number:

Revision Date:

\begin{tabular}{|l|}
\hline$U 15077$ \\
\hline 02 \\
\hline $12 / 17 / 98$ \\
\hline
\end{tabular}

SSA: East-West Drift

Location: East-West Drift

Scenario:

Personnel injury/equipment damage due to falling or partially detached hanging installed equipment (e.g., ventilation ducts, conveyor support framing, power lines, service lines)

Cause, Failure, or Hazardous Event:

Broken support bracket(s), bolts, support stand(s), fasteners. Improper fastening or attaching. Fastener capacity exceeded.

Risk Assignment Before Considering Mitigations:

\begin{tabular}{|c|c|c|}
\hline Frequency Rating: & Consequence Rating: & ${ }^{*}$ Risk Before: \\
\hline A - Frequent & I-Catastrophic & \\
\hline B - Probable & II - Critical & \\
\hline C - Occasional & III - Marginal & High \\
\hline$D$ - Remote & N - Negligible & \\
\hline E - Improbable & & \\
\hline
\end{tabular}

Risk Designation

After

Applying

Mitigations:

\begin{tabular}{|lll|}
\hline Frequency Rating: & Consequence Rating: & Risk After: \\
A - Frequent & I- Catastrophic & \\
B - Probable & III-Critical & Low \\
C - Occasional & III - Marginal & \\
D - Remote & IV - Negligible & \\
E- Improbable & & \\
\hline
\end{tabular}

Related Scenarios:

U10034

Prevention

Mitigation

Documentation: 


\section{DI: BAB000000-01717-0200-0004; Rev 02}

\section{Mitigation Feature}

Scenario Number: U15077 Mitigation Number: 1

Scenario:

Personnel injury/equipment damage due to falling or partially detached hanging installed equipment (e.g., ventilation ducts, conveyor support framing, power lines, service lines)

Mitigation Feature: Design and install brackets, bolts, support framework with adequate safety factors for anticipated loads.

Documentation:

Remarks:

Mitigation type:
Design Feature
Safety Device
Warning Device
Procedure

$\bigcirc$ Training

\section{Mitigation Tracking}

\section{Mitigation \\ Implementation \\ Status:}
Not Implemented
Implemented

Comments:

Implemented (per T.Rotert, aug98.) wp

\section{Appendix A \\ Page UI5077.1}




\section{DI: BAB000000-01717-0200-0004; Rev 02}

\section{Mitigation Feature}

Scenario Number: UI5077 Mitigation Number:

Scenario: $\quad$ Personnel injury/equipment damage due to falling or partially detached hanging installed equipment (e.g., ventilation ducts, conveyor support framing, power lines, service lines)

Mitigation Feature: Minimize access to essential personnel during initial equipment installation and when equipment changes, or repair and maintenance activities are being conducted.

Documentation:

Remarks:

Mitigation type: $\bigcirc$ Design Feature

Safety Device

Warning Device

Procedure

Training

\section{Mitigation Tracking}

Mitigation Implementation

Status:

Comments:
Not Implemented

Implemented

Implemented (per T.Rotert, aug98.) wp

Appendix A

Page UI5077.2 


\section{DI: BAB000000-01717-0200-0004, Rev 02}

\section{Mitigation Feature}

Scenario Number:

U15077

Mitigation Number:

3

Scenario:

Personnel injury/equipment damage due to falling or partially detached hanging installed equipment (e.g., ventilation ducts, conveyor support framing, power lines, service lines)

Mitigation Feature: Shut down any damaged equipment that is still running. Limit access to qualified, trained, and necessary personnel to perform repairs.

Documentation:

Remarks:

Mitigation type:
Design Feature
Safety Device
Warning Device
Procedure
Training

\section{Mitigation Tracking}

Mitigation Implementation Status: Not Implemented Implemented

Comments: $\quad$ Implemented (per T.Rotert, aug98.) wp

Appendix A

Page U15077.3 


\section{DI: BAB000000-01717-0200-0004; Rev 02}

\begin{tabular}{|c|c|}
\hline \multicolumn{2}{|r|}{ Mitigation Feature } \\
\hline Scenario Number: & Mitigation Number: \\
\hline Scenario: & $\begin{array}{l}\text { Personnel injury/equipment damage due to falling or partially detached hanging installed } \\
\text { equipment (e.g., ventilation ducts, conveyor support framing, power lines, service lines) }\end{array}$ \\
\hline Mitigation Feature: & Provide safety training (e.g., personnel exclusion areas, personnel protective equipment) \\
\hline Documentation: & JSAs document. \\
\hline Remarks: & \\
\hline Mitigation type: & $\begin{array}{l}\text { Design Feature } \\
\text { Safety Device } \\
\text { warning Device } \\
\text { Procedure } \\
\text { Training }\end{array}$ \\
\hline
\end{tabular}

\section{Mitigation Tracking}

Mitigation Implementation

Status:

Comments:
Not Implemented

Implemented

Implemented (per T.Rotert, aug98.) wp

\section{Appendix A}

Page UI5077.4 


\section{Scenario Description and Analysis}

\section{Approved}

Scenario Number:

Revision Number:

Revision Date:

Scenario:

Cause, Failure, or Hazardous Event:

Risk Assignment Before Considering Mitigations:

Risk Designation

After

Applying

Mitigations:

Related Scenarios:

Prevention

Mitigation

Documentation:

\begin{tabular}{|l|}
\hline$U 15078$ \\
\hline 02 \\
\hline $12 / 17 / 98$ \\
\hline
\end{tabular}

SSA: $\longdiv { \text { East- West Drift } }$

Location: East-West Drift

Ventilation Fan Failure (Foreign Object(s) in System).

Foreign object(s) ingested into the system.

\begin{tabular}{|lll|}
\hline Frequency Rating: & Consequence Rating: & *Risk Before: \\
A - Frequent & $\bigcirc I$ - Catastrophic & \\
B - Prabable & $\bigcirc I I-$ Critical & Medium \\
C - Occasional & $\bigcirc I I$ - Marginal & \\
D - Remote & $\bigcirc$ IV - Negligible & \\
E - Improbable & & \\
\hline
\end{tabular}

\begin{tabular}{|lll|}
\hline Frequency Rating: & Consequence Rating: & Risk After: \\
A - Frequent & $\bigcirc$ - Catastrophic & \\
B - Probable & $\bigcirc$ & \\
C - Occasional & $O$ - Critical & Low \\
D- Remote & II - Marginal & \\
E- Improbable & & \\
\hline
\end{tabular}

U10105

ESF Subsurface Vaneaxial Ventilation Fans Procurement Specification. Ventilation fan service manual, maintenance manuals and safety manuals. 


\section{DI: BAB000000-01717-0200-0004, Rev 02}

\section{Mitigation Feature}

Scenario Number: $\quad$ U15078 Mitigation Number: 1

Scenario:

Ventilation Fan Failure (Foreign Object(s) in System).

Mitigation Feature:

Install screens upstream of each fan to protect against foreign object damage. The ducting

design should provide access panels adjacent to screen sites for cleaning.

Documentation:

Remarks:

Mitigation type:

Design Feature

Safety Device

Warning Device

Procedure

$O$ Training

\section{Mitigation Tracking}

Mitigation

Implementation

Status:

Comments:
Not Implemented

implemented

Implemented (per T.Rotert, aug98.) wp

Appendix A

Page UI5078.1 


\section{DI: BAB000000-01717-0200-0004; Rev 02}

\section{Mitigation Feature}

Scenario Number: U15078 Mitigation Number:

Scenario: $\quad$ Ventilation Fan Failure (Foreign Object(s) in System).

Mitigation Feature: Perform maintenance in accordance with manufacturer's recommendation to remove foreign objects from screen filters.

Documentation:

Remarks:

Mitigation type:
Design Feature
Safety Device
Warning Device
Procedure
Training

\section{Mitigation Tracking}

Mitigation

Implementation

Status:

Camments:
Not Implemented

Implemented 


\section{Scenario Description and Analysis}

\section{Approved}

Scenario Number:

Revision Number:

Revision Date:

\begin{tabular}{|l|}
\hline$U / 5080$ \\
\hline 02 \\
\hline $12 / 17 / 98$ \\
\hline
\end{tabular}

SSA: East-West Drift

\section{Scenario:}

Fire Hazards: Conveyor System

Cause, Failure, or Hazardous Event:

Risk Assignment Before Considering Mitigations:

Conveyor belt fire due to friction on bearing, idlers frozen.

Location: East-West Drift

Conveyor belt fire due to friction on beaing, idlers frozen, 2.

\begin{tabular}{|lll|}
\hline Frequency Rating: & Consequence Rating: & *Risk Before: \\
O A - Frequent & I-Catastrophic & \\
B - Probable & II - Critical & Medium \\
C - Occasional & III - Marginal & \\
D-Remote & IV - Negligible & \\
E- Improbable & & \\
\hline
\end{tabular}

Risk Designation

After

Applying

Mitigations:

\begin{tabular}{|lll|}
\hline Frequency Rating: & Consequence Rating: & Risk After: \\
A - Frequent & $O_{I-\text { Catastrophic }}$ & \\
B - Probable & $O_{I}$ - Critical & Low \\
C - Occasional & $O_{I I I-\text { Marginal }}$ & \\
D - Remote & $O_{\text {IV - Negligible }}$ & \\
E - Improbable & & \\
\hline
\end{tabular}

Related Scenarios:

\section{U10067}

Prevention

Mitigation

Documentation:

OSHA - 29 CFR 1926 Subparts S \& F. NFPA Fire Protection Rules and Regulations. System specifications, maintenance and safety manuals. 
DI: BAB000000-01717-0200-0004; Rev 02

\section{Mitigation Feature}

Scenario Number:

U15080 Mitigation Number: 1

Scenario:

Fire Hazards: Conveyor System

Mitigation Feature: Install automatic fire suppression systems at all transfer points.

Documentation:

Remarks:

Mitigation type:

Design Feature

Safety Device

Warning Device

Procedure

Training

\section{Mitigation Tracking}

Mitigation

Implementation

Status:

Comments:
Not Implemented

Implemented

Implemented (per T.Rotert, aug98.) wp

\section{Appendix A}

Page UI5080.1 


\section{DI: BAB000000-01717-0200-0004; Rev 02}

\section{Mitigation Feature}

Scenario Number: 415080 Mitigation Number: 2

Scenario: $\quad$ Fire Hazards: Conveyor System

Mitigation Feature: Install fire resistant belts.

Documentation:

Remarks:

Mitigation type:

Design Feature

Safety Device

Warning Device

Procedure

Training

\section{Mitigation Tracking}

Mitigation

Implementation

Status:

Comments:
Not Implemented

Implemented

Implemented (per T.Rotert, aug98.) wp

Appendix A

Page UI5080.2 


\section{DI: BAB000000-01717-0200-0004; Rev 02}

\section{Mitigation Feature}

Scenario Number:

UI5080 Mitigation Number:

Scenario:

Fire Hazards: Conveyor System

Mitigation Feature: $\sqrt{\text { Provide wall mounted fire extinguishers at each electric panel site and every } 200-300 \text { feet on }}$ the tunnel wall.

Documentation:

Remarks:

Mitigation type:
Design Feature
Safety Device
Warning Device
Procedure
Training

\section{Mitigation Tracking}

Mitigation Implementation Status:

Comments:
Not implemented

Implemented 


\section{DI: BAB000000-01717-0200-0004; Rev 02}

\section{Mitigation Feature}

Scenario Number: $415080 \quad$ Mitigation Number: 4

Scenario: $\quad$ Fire Hazards: Conveyor System

Mitigation Feature: Establish inspection, maintenance procedures and schedule for conveyor components.

Maintain inspection and maintenance records.

Documentation:

Remarks:

Mitigation type:
Design Feature
Safety Device
Warning Device
Procedure
Training

\section{Mitigation Tracking}

\section{Mitigation Implementation Status:}

Comments:
Not Implemented

implemented

Implemented (per T. Rotert, aug98_Inspected on shift basis per manufacturers instruction during operations and annual suppression system inspections.) wp. Will be formally documented in the Maximo system. [Bob Law 05/19/99] JLR

\section{Appendix A \\ Page UI5080.4}




\section{Scenario Description and Analysis}

\section{Approved}

Scenario Number:

Revision Number:

Revision Date:

\begin{tabular}{|l|}
\hline$U 15081$ \\
\hline 02 \\
\hline $12 / 17 / 98$ \\
\hline
\end{tabular}

SSA: East-West Drift

Scenario:

Fire Hazards: Ventilation System

Cause, Failure, or Hazardous Event:

Risk Assignment Before Considering Mitigations:

Fire in a fan motor. Friction heat in bearing, electrical short.

Location: East-West Drift

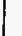

\begin{tabular}{|lll|}
\hline Frequency Rating: & Consequence Rating: & *Risk Before: \\
A - Frequent & $O_{\text {I-Catastrophic }}$ & \\
B - Probable & II-Critical & Medium \\
C - Occasional & III - Marginal & \\
D - Remote & $\bigcirc$ IV - Negligible & \\
E - Improbable & & \\
\hline
\end{tabular}

Risk Designation

After

Applying

Mitigations:

Frequency Rating:
A - Frequent
B - Probable
C - Occasional
D - Remote
E - Improbable

Consequence Rating:

Risk After:

I - Catastrophic

II - critical

III - Marginal

IV - Negligible

Extremely Low

E - Improbable

Related Scenarios:

U10104

Prevention

Mitigation

Documentation:

ESF Subsurface Vaneaxial Ventilation Fans Procurement Specification. Ventilation fan service manual, operational procedures, maintenance manuals and safety manuals.

Appendix A

Page UI5081 


\section{DI: BAB000000-01717-0200-0004, Rev 02}

\section{Mitigation Feature}

Scenario Number: Ui5081 Mitigation Number: 1

Scenario: $\quad$ Fire Hazards: Ventilation System

Mitigation Feature: Metal housing contains fire.

Documentation:

Remarks:

Mitigation type:

Design Feature

Safety Device

Warning Device

Procedure

Q Training

\section{Mitigation Tracking}

Mitigation

Implementation

Status:

Comments:
Not Implemented

Implemented

Implemented (per T.Rotert, aug98.) wp

Appendix A

Page UI5081.1 


\section{DI: BAB000000-01717-0200-0004, Rev 02}

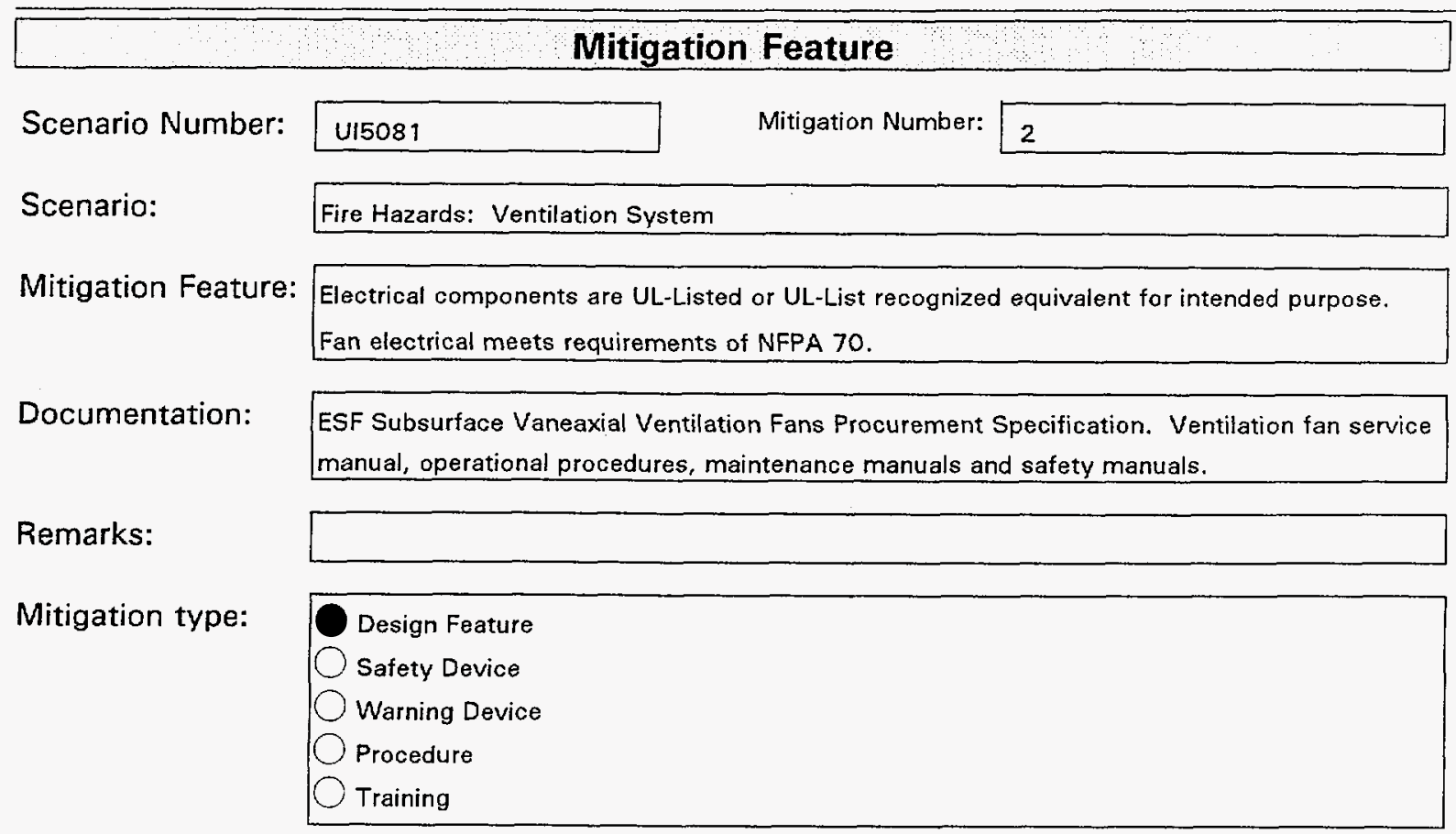

\section{Mitigation Tracking}

Mitigation Implementation Status:

Comments:
Not Implemented

Implemented

Implemented (per T.Rotert, aug98.) wp

\section{Appendix A}

Page UI5081.2 
DI: BAB000000-01717-0200-0004, Rev 02

\section{Mitigation Feature}

Scenario Number: $415081 \quad$ Mitigation Number: 3

Scenario:

Fire Hazards: Ventilation System

Mitigation Feature:

The temperature of each fan's bearings can be inspected for overheating condition.

Documentation:

Remarks:

Mitigation type:

Design Feature

Safety Device

Warning Device

Procedure

Training

Mitigation Tracking

Mitigation

Implementation

Status:

Not Implemented

implemented

Comments:

\section{Appendix A}

Page UI5081.3 


\section{DI: BAB000000-01717-0200-0004; Rev 02}

\section{Mitigation Feature}

Scenario Number: UI5081 Mitigation Number: 4

Scenario: $\quad$ Fire Hazards: Ventilation System

Mitigation Feature: Individual fans can be electrically isolated (e.g., shutoff switch)

Documentation:

Remarks:

Mitigation type:

Design Feature

Safety Device

Warning Device

Procedure

Training

\section{Mitigation Tracking}

Mitigation

Implementation

Status:

Not Implemented

Implemented

Comments: 


\section{DI: BAB000000-01717-0200-0004; Rev 02}

\section{Mitigation Feature}

Scenario Number: U15081 Mitigation Number: 5

Scenario: $\quad$ Fire Hazards: Ventilation System

Mitigation Feature: Combustible materials available are extremely limited.

Documentation:

Remarks:

Mitigation type:

Design Feature

Safety Device

Warning Device

Procedure

Training

\section{Mitigation Tracking}

Mitigation

Implementation

Status:

Comments:
Not Implemented
Implemented

Implemented (per T.Rotert, aug98.) wp

\section{Appendix A}

Page UI5081.5 


\section{Scenario Description and Analysis}

\section{Approved}

Scenario Number:

Revision Number:

Revision Date:

Scenario:

Cause, Failure, or Hazardous Event:

Risk Assignment

Before Considering

Mitigations:

Risk Designation

After

Applying

Mitigations:

Prevention

Mitigation

Documentation:

\begin{tabular}{|l|}
\hline UI5082 \\
\hline 02 \\
\hline $12 / 17 / 98$ \\
\hline
\end{tabular}

SSA: East-West Drift

Location: East-West Drift

Ventilation System Shutdown Due to Main Power Failure

Power grid interruption (e.g., utility power source, site transformers, circuit breakers, switches)

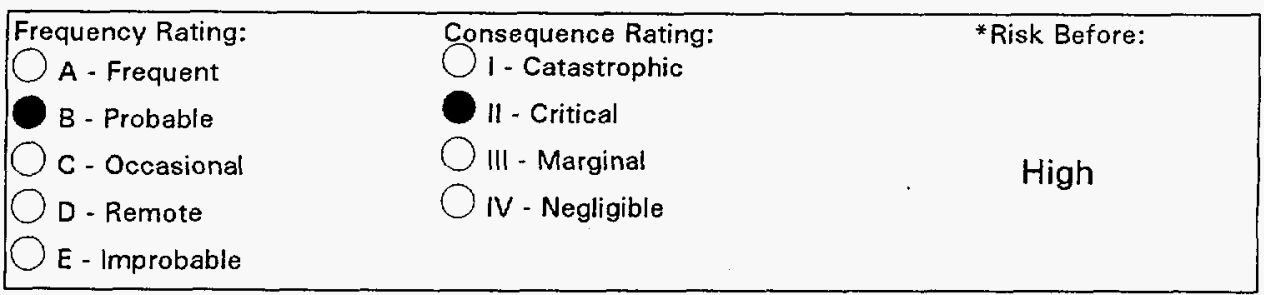

\begin{tabular}{|lll|}
\hline Frequency Rating: & Consequence Rating: & Risk After: \\
A - Frequent & $\bigcirc I-$ Catastrophic & \\
B - Probable & $\bigcirc I I-$ Critical & Low \\
C - Occasional & $\bigcirc \mathrm{III}$ - Marginal & \\
D - Remote & $\bigcirc \mathrm{IV}$ - Negligible & \\
E - Improbable & & \\
\hline
\end{tabular}

U10110

Emergency evacuation plan. Evacuation training and safety manuals. 
DI: BAB000000-01717-0200-0004; Rev 02

\section{Mitigation Feature}

Scenario Number: $\quad$ U15082 Mitigation Number: 1

Scenario: $\quad$ Ventilation System Shutdown Due to Main Power Failure

Mitigation Feature: Develop procedures for cessation of work and evacuation of personnel when ventilation system fails.

Documentation:

Remarks:

Mitigation type:
Design Feature
Safety Device
Warning Device
Procedure
Training

\section{Mitigation Tracking}

Mitigation Implementation Status:

Comments:
Not Implemented

Implemented

Implemented (per T.Rotert, aug98.) wp

\section{Appendix A}

Page UI5082.1 
DI: BAB000000-01717-0200-0004; Rev 02

\section{Mitigation Feature}

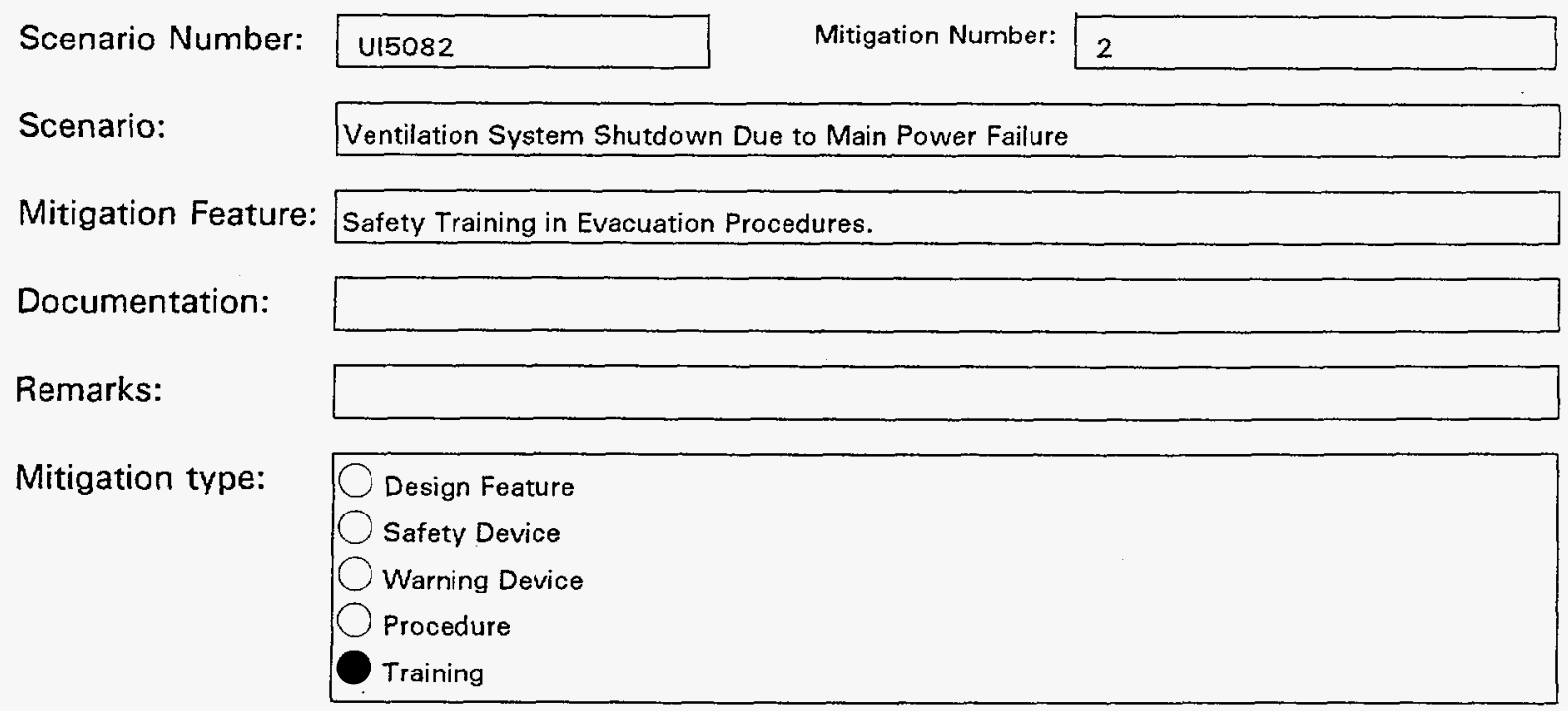

\section{Mitigation Tracking}

Mitigation

Implementation

Status:

Not Implemented

implemented

Comments:

Appendix A

Page UI5082.2 


\section{Scenario Description and Analysis}

\section{Approved}

Scenario Number:

Revision Number:

Revision Date:

Scenario:

Cause, Failure, or Hazardous Event:

Risk Assignment Before Considering Mitigations:

\begin{tabular}{|l|}
\hline 015083 \\
\hline 02 \\
\hline $12 / 17 / 98$ \\
\hline
\end{tabular}

Inadequate Lighting Hazard

Failure of support lighting system.

\begin{tabular}{|c|c|c|}
\hline Frequency Rating: & Consequence Rating: & ${ }^{*}$ Risk Before: \\
\hline A - Frequent & I- Catastrophic & \\
\hline B - Probable & II - Critical & \\
\hline D - Occasional & III - Marginal & High \\
\hline D - Remote & IV - Neglimible & \\
\hline E - Improbable & & \\
\hline
\end{tabular}

Risk Designation

After

Applying

Mitigations:

Frequency Rating:
A - Frequent
B - Probable
C - Occasional
D - Remote
E - Improbable

Related Scenarios:

010242

Prevention

Mitigation

Documentation:
Consequence Rating: Risk After:

1 - Catastrophic

II - Critical

III - Marginal

Extremely Low
SSA: East-West Drift

Location: East-West Drift
OSHA 29 CFR 1926.800 \{1\}. System specifications and design drawings. Maintenance and safety manuals.

Appendix A

Page U15083 


\section{Di: BAB000000-01717-0200-0004; Rev 02}

\section{Mitigation Feature}

Scenario Number: $415083 \quad$ Mitigation Number: 1

Scenario: $\quad$ Inadequate Lighting Hazard

Mitigation Feature: Provide emergency lighting with battery backup at all telephone stations.

Documentation:

Remarks:

Mitigation type:
Design Feature
Safety Device
Warning Device
$\bigcirc$ Procedure
0 Training

\section{Mitigation Tracking}

Mitigation

Implementation

Status:

Comments:
Not Implemented

Implemented

Appendix A

Page UI5083.1 


\section{DI: BAB000000-01717-0200-0004; Rev 02}

\section{Mitigation Feature}

Scenario Number: $U 15083 \quad$ Mitigation Number: 2

Scenario:

Inadequate Lighting Hazard

Mitigation Feature: Provide individual power pack lights/flashlights for emergency use.

Documentation:

Remarks:

Mitigation type:
Design Feature
Safety Device
Warning Device
Procedure
Training

\section{Mitigation Tracking}

Mitigation Implementation Status:

Comments:
Not Implemented

Implemented

\section{Appendix A}

Page UI5083.2 


\section{DI: BAB000000-01717-0200-0004; Rev 02}

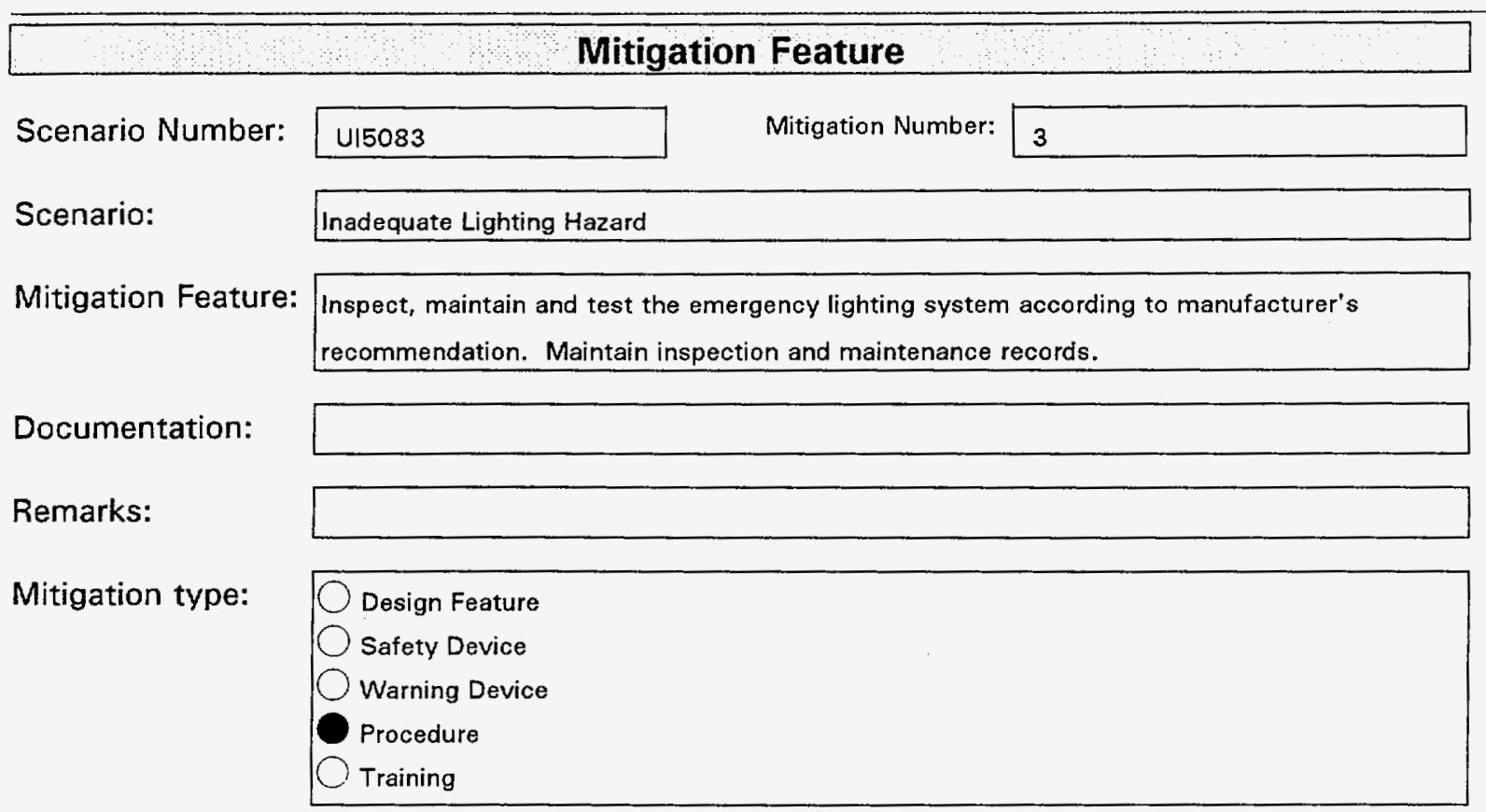

\section{Mitigation Tracking}

Mitigation Implementation Status:

Comments:
Not Implemented

O Implemented 
DI: BAB000000-01717-0200-0004; Rev 02

\begin{tabular}{|c|c|c|}
\hline \multicolumn{3}{|c|}{ Mitigation Feature } \\
\hline Scenario Number: & Mitigation Number: & 4 \\
\hline Scenario: & Inadequate Lighting Hazard & \\
\hline Mitigation Feature: & Provide lighted Exit Signs with battery backup at all inters & ections. \\
\hline Documentation: & $\begin{array}{l}\text { OSHA } 29 \text { CFR } 1926.800(1) \text {. System specifications and } \\
\text { safety manuals. }\end{array}$ & esign drawings. Maintenance and \\
\hline Remarks: & & \\
\hline Mitigation type: & $\begin{array}{l}\text { Design Feature } \\
\text { Safety Device } \\
\text { warning Device } \\
\bigcirc \text { Procedure } \\
\bigcirc \text { Training }\end{array}$ & \\
\hline
\end{tabular}

\section{Mitigation Tracking}

Mitigation

Implementation

Status:

Comments:
Not Implemented

Implemented

Appendix A

Page UI5083.4 


\section{DI: BAB000000-01717-0200-0004, Rev 02}

\section{Scenario Description and Analysis}

\section{Approved}

Scenario Number:

Revision Number:

Revision Date:

Scenario:

Cause, Failure, or Hazardous Event:

Risk Assignment Before Considering Mitigations:

\begin{tabular}{|l|}
\hline U15084 \\
\hline 02 \\
\hline $12 / 17 / 98$ \\
\hline
\end{tabular}

Diesel Fuel Hazard: Leak in Engine compartment

Internal fuel line leak from mechanical failure (joints, intrusive rupture)

\begin{tabular}{|lll|}
\hline Frequency Rating: & Consequence Rating: & "Risk Before: \\
A - Frequent & $\bigcirc$ I- Catastrophic & \\
B - Probable & OII - Critical & Medium \\
C - Occasional & III - Marginal & \\
D - Remote & OIV - Negligible & \\
E- Improbable & & \\
\hline
\end{tabular}

Risk Designation

After

Applying

Mitigations:

Frequency Rating:
A - Frequent
B - Probable
C - Occasional
D - Remote
E - Improbable

Consequence Rating:

Risk After:

I - Catastrophic

OII - Critical

III - Marginal

Extremely Low

Related Scenarios:

U10354

Prevention

Mitigation

Documentation:

NFPA 124, Diesel Fuel and Diesel Equipment in Underground Mines.

NFPA 122, Standard for Storage of Flammable and Combustible Liquids within Underground Metal and Non-Metal Mines.

OSHA - 29 CFR 1926 Subpart S.

Subsurface Fire Hazard Analysis, BABFAH000-01717-0200-00121 Rev. 0

Subsurface Fire Protection Design Analysis, BABFAH000-01717-0200-00114 Rev. 0

Subsurface Fire Protection, BABFAHO00-01717-6300-15300 Rev. 0

Fire Alarm and Smoke Detector System, BABFAHO00-01717-6300-16721 Rev. 0

Emergency evacuation plan and procedures.

Maintenance, operations and housekeeping procedures.

Inspection and maintenance records.

\section{Appendix A}

Page UI5084 


\section{DI: BAB000000-01717-0200-0004; Rev 02}

\section{Mitigation Feature}

Scenario Number:

$$
\text { U15084 }
$$

Mitigation Number:

1

Scenario:

Diesel Fuel Hazard: Leak in Engine compartment

Mitigation Feature: Provide an automatic or manual fire suppression system in all mobile diesel equipment.

Documentation:

Remarks:

Mitigation type:

$$
\begin{aligned}
& \text { Design Feature } \\
& \text { Safety Device } \\
& \text { Warning Device } \\
& \text { Procedure } \\
& \text { Training }
\end{aligned}
$$

\section{Mitigation Tracking}

\section{Mitigation Implementation \\ Status:}

Comments:

\section{Not Implemented \\ Implemented}

Appendix A

Page UI5084.1 


\section{DI: BAB000000-01717-0200-0004; Rev 02}

\section{Mitigation Feature}

Scenario Number:

U15084 Mitigation Number:

2

Scenario:

Diesel Fuel Hazard: Leak in Engine compartment

Mitigation Feature: Engine compartment is an enclosed space.

Documentation:

Remarks:

[Note: When written, this mitigation was intended for the locomotive. wprather]

Mitigation type:

$\bigcirc$ Safety Device

Warning Device

Procedure

Training

\section{Mitigation Tracking}

Mitigation Implementation Status:

Not Implemented

Implemented

Comments:

Implemented (per T.Rotert, aug98_Locomotive is enclosed, not possible on some other equipment.) wp

Appendix A

Page UI5084.2 


\section{DI: BAB000000-01717-0200-0004, Rev 02}

\section{Mitigation Feature}

Scenario Number:

UI5084 Mitigation Number:

3

Scenario:

Diesel Fuel Hazard: Leak in Engine compartment

Mitigation Feature: Shut down diesel engine at first sign of any leak or spill condition. Clean or repair on site.

Documentation:

Remarks:

Mitigation type:
$\bigcirc$ Design Feature
Safety Device
$\bigcirc$ Warning Device
Procedure
Training

\section{Mitigation Tracking}

Mitigation Implementation Status:

Comments:
$($ Not Implemented
Implemented

\section{Appendix A}

Page UI5084.3 
DI: BAB000000-01717-0200-0004, Rev 02

\section{Scenario Description and Analysis}

\section{Approved}

Scenario Number:

Revision Number:

Revision Date:

Scenario:

Cause, Failure, or Hazardous Event:

Risk Assignment

Before Considering Mitigations:

\begin{tabular}{|l|}
\hline U15085 \\
\hline 02 \\
\hline $12 / 17 / 98$ \\
\hline
\end{tabular}

Vehicle hits personnel on foot in the East-West Drift.

Vehicle operating in the East-West Drift runs into personnel working/walking in the Drift (walkway/tracks accupy same transit window as vehiclel.

\begin{tabular}{|lll|}
\hline Frequency Rating: & Consequence Rating: & *Risk Before: \\
A - Frequent & I - Catastrophic & \\
B - Probable & II - Critical & High \\
C - Occasional & $\bigcirc I I-$ Marginal & \\
D - Remote & $\bigcirc N$ - Negligible & \\
E- Improbable & & \\
\hline
\end{tabular}

Risk Designation

After

Applying

Mitigations:

\begin{tabular}{|lll|}
\hline Frequency Rating: & Consequence Rating: & Risk After: \\
A - Frequent & I- Catastrophic & \\
B - Probable & OII-Critical & Low \\
C - Occasional & OII - Marginal & \\
D- Remote & OIV - Negligible & \\
E - Improbable & & \\
\hline
\end{tabular}

Related Scenarios:

\section{U10207}

It is the constructor's and operator's responsibility to assure that the hazard(s) associated with this scenario have been mitigated through proper documentation
Prevention

Mitigation

Documentation:
Appendix A

Page U15085 


\section{DI: BAB000000-01717-0200-0004; Rev 02}

\section{Mitigation Feature}

$\begin{array}{ll}\text { Scenario Number: } & \text { Ul5085 } \\ \text { Scenario: } & \text { Vehicle hits personnel on foot in the East-West Drift. } \\ \text { Mitigation Feature: } & \text { Install warning devices (flashing lights/sounding horn) on vehicles to warn personnel of vehicle } \\ \text { approach. }\end{array}$

\section{Mitigation Tracking}

Mitigation Implementation Status:

Comments:
Not Implemented

Implemented

Implemented (per T.Rotert, aug98_Locomotives are equiped.) wp

\section{Appendix A}

Page U15085.1 


\section{DI: BAB000000-01717-0200-0004; Rev 02}

\section{Mitigation Feature}

Scenario Number: U15085 Mitigation Number:

Scenario: $\quad$ Vehicle hits personnel on foot in the East-West Drift.

Mitigation Feature: Provide personnel working/walking between the ECRB entrance and the TBM complex (e.g. TBM, support cars/structures, support equipment) with reflective vests, reflective tape on hard hats and lights (e.g., cap lamps, flashlights) to signal vehicle operator of their presence.

Documentation:

It is the constructor's and operator's responsibility to assure that the hazard(s) associated with this scenario have been mitigated through proper documentation

Remarks:

Mitigation type:
Design Feature
Safety Device
Warning Device
Procedure
Craining

\section{Mitigation Tracking}

Mitigation

Implementation

Status:

Comments:
Not Implemented

Implemented 


\section{DI: BAB000000-01717-0200-0004, Rev 02}

\begin{tabular}{|c|c|}
\hline$:$ & Mitigation Feature \\
\hline Scenario Number: & Mitigation Number: \\
\hline Scenario: & Vehicle hits personnel on foot in the East-West Drift. \\
\hline Mitigation Feature: & $\begin{array}{l}\text { Provide flat jump seat areas mounted on top of the top utility pipe spaced to allow quick } \\
\text { access when a train is approaching (e.g., co-located with the fire extinguishers). The seat } \\
\text { must allow for complete relief from the train travel area. }\end{array}$ \\
\hline Documentation: & $\begin{array}{l}\text { It is the constructor's and operator's responsibility to assure that the hazard(s) associated } \\
\text { with this scenario have been mitigated through proper documentation }\end{array}$ \\
\hline Remarks: & \\
\hline Mitigation type: & $\begin{array}{l}\text { Design Feature } \\
\text { Safety Device } \\
\text { Warning Device } \\
\text { Procedure } \\
\text { Training }\end{array}$ \\
\hline
\end{tabular}

\section{Mitigation Tracking}

\section{Mitigation Implementation Status:}

Comments:
Not Implemented

Implemented 


\section{DI: BAB000000-01717-0200-0004, Rev 02}

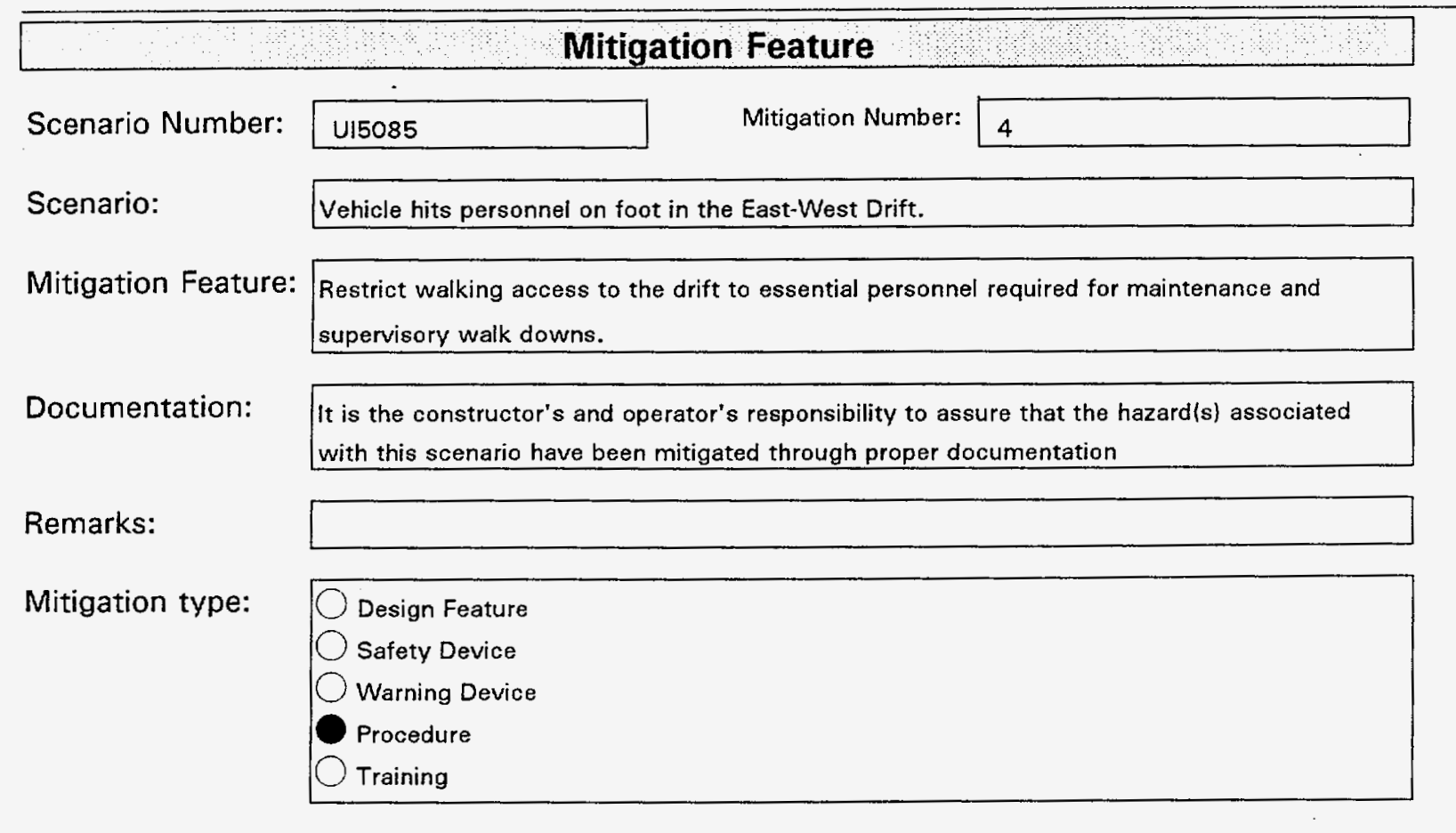

\section{Mitigation Tracking}

\section{Mitigation Implementation Status:}

Comments:
Not Implemented
Implemented

Implemented (per T.Rotert, aug98.) wp

\section{Appendix A \\ Page UI5085.4}




\section{DI: BAB000000-01717-0200-0004; Rev 02}

\section{Mitigation Feature}

$\begin{array}{ll}\text { Scenario Number: } & \text { U15085 } \\ \text { Scenario: } & \text { Vehicle hits personnel on foot in the East-West Drift. } \\ \text { Mitigation Feature: } & \begin{array}{l}\text { Train traffic will be suspended during special walk downs/inspections (e.g., DIE, Safety, or } \\ \text { other special purpose walk downs). }\end{array}\end{array}$

Documentation: It is the constructor's and operator's responsibility to assure that the hazard(s) associated with this scenario have been mitigated through proper documentation

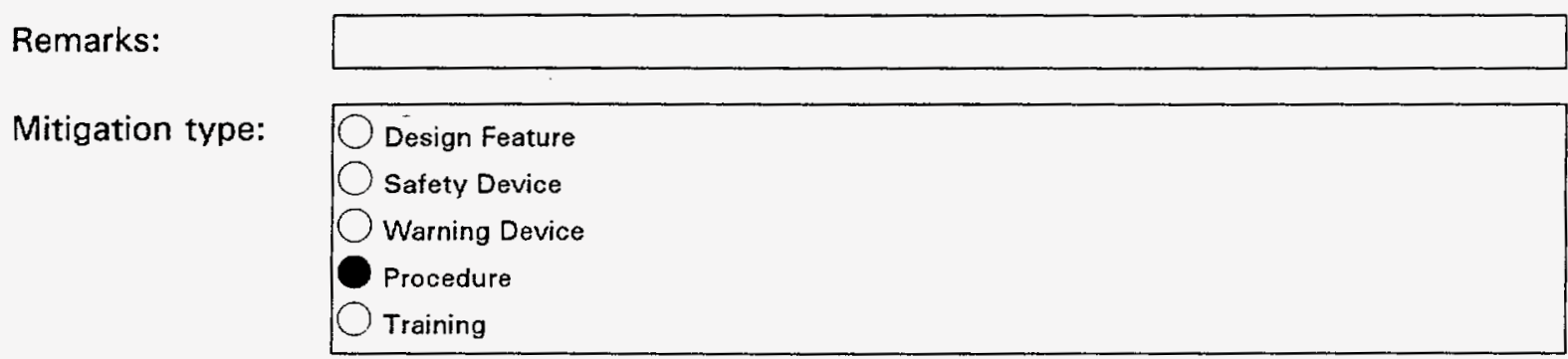

\section{Mitigation Tracking}

\section{Mitigation Implementation Status:}

Comments:
Not Implemented

implemented

Implemented (per T.Rotert, aug98.) wp

\section{Appendix A}

Page U15085.5 


\section{DI: BAB000000-01717-0200-0004; Rev 02}

\begin{tabular}{l}
\hline Scenario Number: \\
Scenario: \\
Mitigation Feature: $\begin{array}{ll}\text { Dehicle hits personnel on foot in the East-West Drift. } \\
\text { extended periods in the train travel area. }\end{array}$ \\
Documentation: \\
Remarks: $\begin{array}{l}\text { It is the constructor's and operator's responsibility to assure that the hazard(s) associated } \\
\text { with this scenario have been mitigated through proper documentation }\end{array}$ \\
Mitigation type: \\
$\begin{array}{l}\text { Design Feature } \\
\text { Safety Device } \\
\text { Warning Device } \\
\text { Procedure } \\
\text { Training }\end{array}$ \\
\hline
\end{tabular}

\section{Mitigation Tracking}

Mitigation Implementation

Status:

Comments:

\section{Not Implemented}

Implemented

Implemented (per T.Rotert, aug98,) wp

\section{Appendix A \\ Page UI5085.6}


DI: BAB000000-01717-0200-0004, Rev 02

\section{Mitigation Feature}

Scenario Number: U15085 Mitigation Number:

Scenario: Vehicle hits personnel on foot in the East-West Drift.

Mitigation Feature: Loci operators will be trained to bring the trains to a stop until personnel on foot in the tunnel are safely seated on a jump seat (Mitigation 3 ) before proceeding.

Documentation: It is the constructor's and operator's responsibility to assure that the hazard(s) associated with this scenario have been mitigated through proper documentation

Remarks:

Mitigation type:
Design Feature
Safety Device
Warning Device
Procedure
Training

\section{Mitigation Tracking}

Mitigation Implementation Status:

Comments:

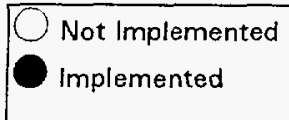

Implemented (per T. Rotert, aug98) wp.

\section{Appendix A}

Page UI5085.7 


\section{DI: BAB000000-01717-0200-0004; Rev 02}

\section{Mitigation Feature}

$\begin{array}{ll}\text { Scenario Number: } & \text { U15085 } \\ \text { Scenario: } & \text { Vehicle hits personnel on foot in the East-West Drift. } \\ \text { Mitigation Feature: } & \text { Train personnel to proceed to the nearest jump seat when a train is approaching. } \\ \text { Documentation: } & \text { It is the constructor's and operator's responsibility to assure that the hazard(s) associated } \\ \text { with this scenario have been mitigated through proper documentation } \\ \text { Remarks: } \\ \text { Mitigation type: } \\ \text { Design Feature } \\ \text { Safety Device } \\ \text { Warning Device } \\ \text { Procedure } \\ \text { Training }\end{array}$

\section{Mitigation Tracking}

Mitigation Implementation Status:

Not implemented

Implemented

Comments: Implemented (per T. Rotert, aug98) wp.

Appendix A

Page U15085.8 


\section{DI: BAB000000-01717-0200-0004; Rev 02}

\section{Mitigation Feature}

Scenario Number:

U15085

Mitigation Number:

9

Scenario:

Vehicle hits personnel on foot in the East-West Drift.

Mitigation Feature:

Restrict general access to the drift to authorized personnel only le.g., require special badges

to enter the drift).

Documentation: It is the constructor's and operator's responsibility to assure that the hazard(s) associated with this scenario have been mitigated through proper documentation

Remarks:

Mitigation type:
Design Feature
Safety Device
Warning Device
Procedure
Training

\section{Mitigation Tracking}

\section{Mitigation Implementation Status:}

Comments:

Not Implemented
Implemented

Implemented (per T.Rotert, aug98.) wp

\section{Appendix A \\ Page UI5085.9}




\section{DI: BAB000000-01717-0200-0004; Rev 02}

\begin{tabular}{|c|c|c|}
\hline \multicolumn{3}{|c|}{ Mitigation Feature } \\
\hline Scenario Number: & Mitigation Number: & 10 \\
\hline Scenario: & \multicolumn{2}{|l|}{ Vehicle hits personnel on foot in the East-West Drift. } \\
\hline Mitigation Feature: & \multicolumn{2}{|c|}{$\begin{array}{l}\text { Whenever a train or other vehicle encounters personnel working or walking in the ECRB, the } \\
\text { operator shall bring the train to a complete stop before proceeding. This stop will be } \\
\text { accomplished even if on foot personnel are already prepared for vehicle passage and are well } \\
\text { clear of the vehicle path. }\end{array}$} \\
\hline Documentation: & & \\
\hline Remarks: & & \\
\hline Mitigation type: & $\begin{array}{l}\text { Design Feature } \\
\text { Safety Device } \\
\text { Warning Device } \\
\text { Procedure } \\
\text { Training }\end{array}$ & \\
\hline
\end{tabular}

\section{Mitigation Tracking}

Mitigation

Implementation

Status:

Comments:
Not Implemented

$\bigcirc$ implemented

Appendix A

Page Ui5085.10 


\section{Scenario Description and Analysis}

\section{Approved}

Scenario Number:

Revision Number:

Revision Date:

\section{Scenario:}

Cause, Failure, or Hazardous Event:

\section{Risk Assignment Before Considering Mitigations:}

\begin{tabular}{|l|}
\hline$U / 5086$ \\
\hline 02 \\
\hline $12 / 17 / 98$ \\
\hline
\end{tabular}

Vehicle Diesel Fire Hazard: Diesel leak(s) from fuel tank or fuel lines or spills during refueling.

Mechanical failure(s) of container, fuel lines/joints. Human error during refueling activities (dropping container, inattention to refueling process).

\begin{tabular}{|lll|}
\hline Frequency Rating: & Consequence Rating: & "Risk Before: \\
A - Frequent & I-Catastrophic & \\
B - Probable & $\bigcirc$ - Critical & Medium \\
C - Occasional & $\bigcirc \mathrm{N}$ - Negligible & \\
D-Remote & & \\
E- Improbable & & \\
\hline
\end{tabular}

\section{Risk Designation \\ After \\ Applying \\ Mitigations:}

Related Scenarios:

Prevention

Mitigation

Documentation:

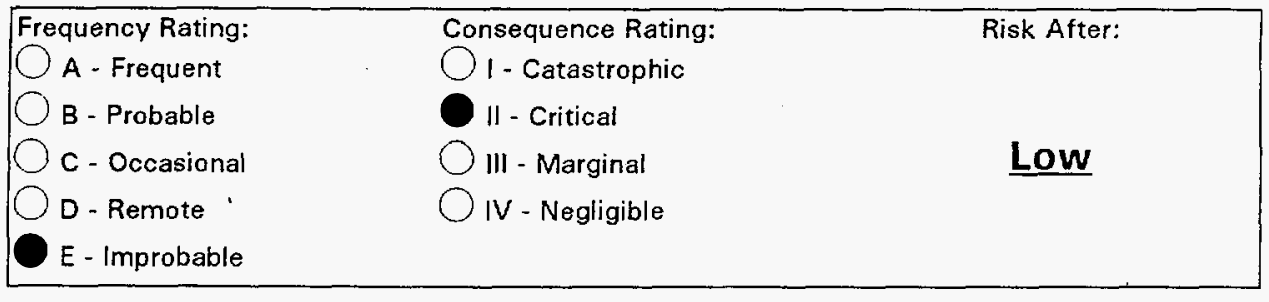

\section{U10361}

NFPA 124, Diesel Fuel and Diesel Equipment in Underground Mines.

NFPA 122, Standard for Storage of Flammable and Combustible Liquids within Underground Metal and Non-Metal Mines.

OSHA - 29 CFR 1926 Subpart S.

Subsurface Fire Hazard Analysis, BABFAHOO0-01717-0200-00121 Rev. O

Subsurface Fire Protection Design Analysis, BABFAH000-01717-0200-00114 Rev. 0

Subsurface Fire Protection, BABFAHOO0-01717-6300-15300 Rev. 0

Fire Alarm and Smoke Detector System, BABFAHOO0-01717-6300-16721 Rev. O

Emergency evacuation plan and procedures.

Maintenance, operations and housekeeping procedures.

Inspection and maintenance records.

\section{Appendix A \\ Page U15086}




\section{DI: BAB000000-01717-0200-0004; Rev 02}

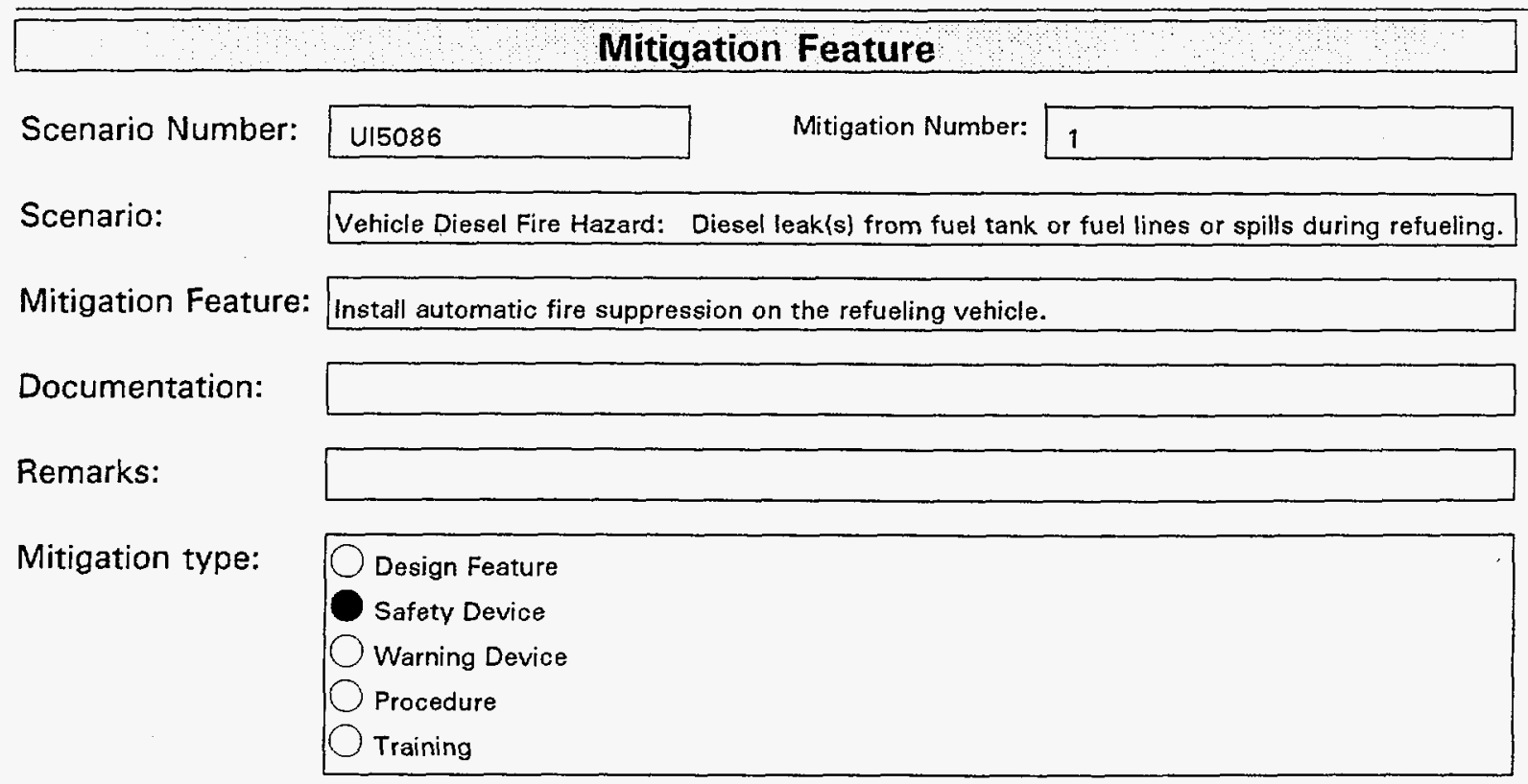

\section{Mitigation Tracking}

Mitigation Implementation Status:

Comments:
Not Implemented

Implemented 


\section{DI: BAB000000-01717-0200-0004, Rev 02}

\section{Mitigation Feature}

$\begin{array}{ll}\text { Scenario Number: } & \text { Ul5086 } \\ \text { Scenario: } & \text { Mehicle Diesel Fire Hazard: Diesel leak (s) from fuel tank or fuel lines or spills during refueling. } \\ \text { Mitigation Feature: } & \text { Daily inspection of diesel engine compartment and fuel lines to detect any small leaks. } \\ \text { Documentation: } & \\ \text { Remarks: } & \\ \text { Mitigation type: } & \\ & \text { Design Feature } \\ & \text { Safety Device } \\ & \text { Warning Device } \\ & \text { Procerure } \\ & \text { Training } \\ & \end{array}$

\section{Mitigation Tracking}

Mitigation Implementation Status: Not Implemented implemented

Comments: Implemented (per T.Rotert, aug98_Part of the pre-shift inspection.) wp

Appendix A

Page UI5086.2 
DI: BAB000000-01717-0200-0004; Rev 02

\section{Mitigation Feature}

Scenario Number: U15086 Mitigation Number:

Scenario: $\quad$ Vehicle Diesel Fire Hazard: Diesel leak (s) from fuel tank or fuel lines or spills during refueling.

Mitigation Feature: All diesel refueling activities shall be a two man operation. One refuels and the other mans the fire extinguisher and diesel fuel cutoff valve.

Documentation:

Remarks:

Mitigation type:
Design Feature
Safety Device
Warning Device
Procedure
Training

\section{Mitigation Tracking}

Mitigation Implementation Status:

Comments:
Not Implemented

Implemented

Appendix A

Page U15086.3 


\section{DI: BAB000000-01717-0200-0004, Rev 02}

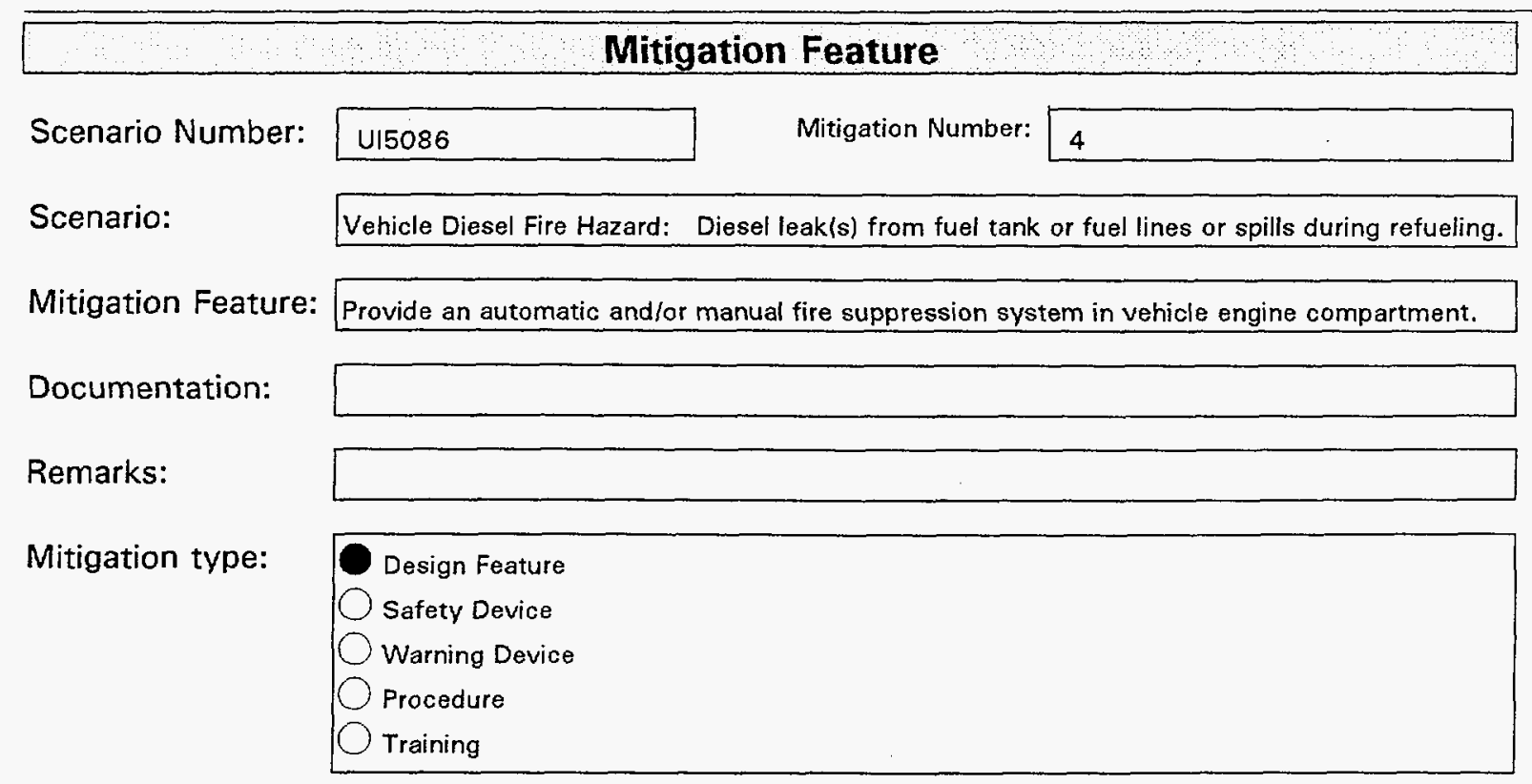

\section{Mitigation Tracking}

Mitigation

Implementation

Status:

Not Implemented

Implemented

Comments:

Implemented (per T.Rotert, aug98.) wp

Appendix A

Page UI5086.4 


\section{DI: BAB000000-01717-0200-0004; Rev 02}

\section{Mitigation Feature}

Scenario Number:

U15086

Mitigation Number:

5

Scenario:

Vehicle Diesel Fire Hazard: Diesel leak(s) from fuel tank or fuel lines or spills during refueling.

Mitigation Feature: Operator training on proper methods of handling diesel fuel and refueling procedures.

Documentation:

Remarks:

Mitigation type:

$$
\begin{aligned}
& \text { Design Feature } \\
& \text { Safety Device } \\
& \text { Warning Device } \\
& \text { Procedure } \\
& \text { Training }
\end{aligned}
$$

\section{Mitigation Tracking}

Mitigation Implementation Status: Not Implemented

implemented

Comments: $\quad$ Implemented (per T.Rotert, aug98.) wp

Appendix A

Page UI5086.5 


\section{Scenario Description and Analysis}

\section{Approved}

Scenario Number:

Revision Number:

Revision Date:

\section{Scenario:}

Cause, Failure, or Hazardous Event:

Risk Assignment Before Considering Mitigations:

Risk Designation After

Applying

Mitigations:

Related Scenarios:

Prevention

Mitigation

Documentation:

\begin{tabular}{|l|}
\hline$U 15087$ \\
\hline 02 \\
\hline $12 / 17 / 98$ \\
\hline
\end{tabular}

\author{
SSA: East-West Drift \\ Location: East-West Drift
}

Train/locomotive derailment and/or impacting the TBM causing personnel injury and/or major equipment damage.

Track failure (e.g., track damage, debris on track). Component failure (e.g., brakes, runaway locomotive). Operator error (e.g., excessive speed, misjudgment or failure to stop causing collision with other equipment). Operator incapacitated.

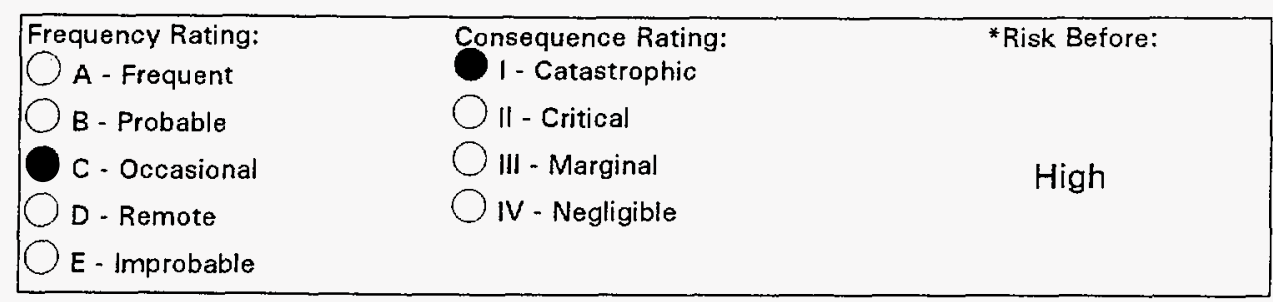

\begin{tabular}{|lll|}
\hline Frequency Rating: & Consequence Rating: & Risk After: \\
A - Frequent & I- Catastrophic & \\
B - Probable & $\bigcirc I I-$ Critical & Low \\
C - Occasional & $\bigcirc I I I-$ Marginal & \\
D- Remote & $\bigcirc I V-$ Negligible & \\
E - Improbable & & \\
\hline
\end{tabular}

U10201, U10202

Title II Design Drawings, Vehicle and Equipment Specifications. OSHA - 29 CFR 1926.800

Operators and maintenance manuals.

Training and safety manuals.

\section{Appendix A \\ Page U15087}




\section{DI: BAB000000-01717-0200-0004; Rev 02}

\section{Mitigation Feature}

Scenario Number:

U15087

Mitigation Number:

1

Scenario:

Train/locomotive derailment and/or impacting the TBM causing personnel injury and/or major equipment damage.

Mitigation Feature: Incorporated "deadman" controls and an emergency stop switch in locomotive.

Documentation:

Remarks:

Mitigation type: $\quad$ Design Feature

Safety Device

Warning Device

Procedure

Training

\section{Mitigation Tracking}

Mitigation

Implementation

Status:

Not Implemented

Implemented

Comments:

Implemented (per T.Rotert, aug98_Deadman in place.) wp

\section{Appendix A}

Page UI5087.1 


\section{DI: BAB000000-01717-0200-0004, Rev 02}

\section{Mitigation Feature}

Scenario Number:

U15087

Mitigation Number:

2

Scenario:

Train/locomotive derailment and/or impacting the TBM causing personnel injury and/or major equipment damage.

Mitigation Feature: Install a speedometer on the locomotive.

Documentation:

Remarks:

Mitigation type: $\bigcirc$ Design Feature

Safety Device

Warning Device

Procedure

Training

\section{Mitigation Tracking}

Mitigation

Implementation

Status:

Comments:
Not Implemented

Implemented

Implemented (per T.Rotert, aug98.) wp

Appendix A

Page UI5087.2 


\section{DI: BAB000000-01717-0200-0004, Rev 02}

\section{Mitigation Feature}

Scenario Number:

Scenario:

Train/locomotive derailment and/or impacting the TBM causing personnel injury and/or major equipment damage.

Mitigation Feature: Install derail devices and/or bumper blocks behind the mapping floor and TBM work areas.

Documentation:

Remarks:

Mitigation type:

$$
\begin{aligned}
& \text { Design Feature } \\
& \text { Safety Device } \\
& \text { Warning Device } \\
& \text { Procedure } \\
& \text { Training }
\end{aligned}
$$

\section{Mitigation Tracking}

Mitigation Implementation

Status:

Comments:
Not Implemented

implemented

Implemented (per T.Rotert, aug98.) wp

Appendix A

Page UI5087.3 


\section{DI: BAB000000-01717-0200-0004, Rev 02}

\section{Mitigation Feature}

Scenario Number: UI5087 Mitigation Number:

Scenario:

Train/locomotive derailment and/or impacting the TBM causing personnel injury and/or major equipment damage.

Mitigation Feature: Test the function of the braking system daily.

Documentation:

Remarks:

Mitigation type:
Design Feature
Safety Device
Warning Device
Procedure
Training

\section{Mitigation Tracking}

\section{Mitigation Implementation Status:}

Comments:

\section{Not Implemented}

Implemented

Implemented (per T.Rotert, aug98 Part of the pre-shift inspection.) wp

Appendix A

Page UI5087.4 


\section{DI: BAB000000-01717-0200-0004; Rev 02}

\section{Mitigation Feature}

Scenario Number:

U15087 Mitigation Number:

5

Scenario:

Train/locomotive derailment and/or impacting the TBM causing personnel injury and/or major equipment damage.

Mitigation Feature: Daily inspection of track to detect any damage and to ensure that their no

equipment/structure and or support system(s) intruding into the transit envelop.

Documentation:

Remarks:

Mitigation type:
Design Feature
Safety Device
Warning Device
Procedure
Training

\section{Mitigation Tracking}

Mitigation Implementation

Status:

Not Implemented

implemented

Comments:

\section{Appendix A}

Page UI5087.5 


\section{DI: BAB000000-01717-0200-0004; Rev 02}

\section{Mitigation Feature}

Scenario Number: $\quad$ UI5087 Mitigation Number: 6

Scenario:

Train/locomotive derailment and/or impacting the TBM causing personnel injury and/or major equipment damage.

Mitigation Feature: Operating contractor will establish and enforce a safe maximum speed limit for the lacomotive in the tunnel.

Documentation:

Remarks:

Mitigation type:
Design Feature
Safety Device
Warning Device
Procedure
Training

\section{Mitigation Tracking}

Mitigation

Implementation

Status:

Not Implemented

Implemented

Comments:

Implemented (per T.Rotert, aug98_Speed limit of $15 \mathrm{mph}$ for locomotive traffic set by CMO and constructor.) wp

\section{Appendix A \\ Page UI5087.6}




\section{DI: BAB000000-01717-0200-0004; Rev 02}

\section{Mitigation Feature}

Scenario Number:

U15087

Mitigation Number:

7

Scenario:

Train/locomotive derailment and/or impacting the TBM causing personnel injury and/or major equipment damage.

Mitigation Feature: Locomotive operator training. Contractor will insure that all locomotive operators are properly trained and qualified.

Documentation:

Remarks:

Mitigation type:
Design Feature
Safety Device
Warning Device
Procedure
Training

\section{Mitigation Tracking}

Mitigation

Implementation

Status:

Comments:
Not Implemented

Implemented 


\section{DI: BAB000000-01717-0200-0004; Rev 02}

\section{Mitigation Feature}

Scenario Number: $\quad$ U15087 Mitigation Number: 8

Scenario: $\quad$ Train/locomotive derailment and/or impacting the TBM causing personnel injury and/or major equipment damage.

Mitigation Feature:

Accomplish daily equipment inspections and remove from sevice ("red tag") any equipment that does not conform with safety requirements.

Documentation: Title II Design Drawings, Vehicle and Equipment Specifications.

OSHA - 29 CFR 1926.800

Operators and maintenance manuals.

Training and safety manuals.

Remarks:

Mitigation type: $\bigcirc$ Design Feature

Safety Device

Warning Device

Procedure

Training

\section{Mitigation Tracking}

Mitigation Implementation

Status:

Comments:
Not Implemented

Implemented

Appendix A

Page UI5087.8 


\section{Scenario Description and Analysis}

\section{Approved}

Scenario Number:

Revision Number:

Revision Date:

\section{Scenario:}

Cause, Failure, or Hazardous Event:

Risk Assignment Before Considering Mitigations:

\begin{tabular}{|l|}
\hline$U 15089$ \\
\hline 02 \\
\hline $12 / 17 / 98$ \\
\hline
\end{tabular}

Personnel working on the mapping floor come into contact with operating muck conveyor system mechanical parts (e.g., conveyor belts, idlers, etc.)

Personnel inadvertently walk into or back into the running conveyor system. Personnel reach into or bend forward into the conveyor envelope to survey the tunnel walls or retrieve tools/materials from conveyor side of the mapping floor. Personnel fall into the conveyor system from mobile or raised stands (e.g., ladders, stools, etc.) on the mapping floor.

\begin{tabular}{|lll|}
\hline Frequency Rating: & Consequence Rating: & *Risk Before: \\
A - Frequent & I - Catastrophic & \\
B - Probable & II - Critical & High \\
C - Occasional & III - Marginal & \\
D - Remote & IV - Negligible & \\
E- Improbable & & \\
\hline
\end{tabular}

Risk Designation

After

Applying

Mitigations:

Frequency Rating:
A - Frequent
B - Probable
C - Occasional
D - Remote
E - Improbable

Related Scenarios:

Prevention

Mitigation

Documentation:
Consequence Rating:

Risk After:

O1 - Catastrophic

II - Critical

III - Marginal

Low
- Improbable

Underground mapping of the ECRB JSA, 19jun98,page1.

Appendix A

Page UI5089 


\section{DI: BAB000000-01717-0200-0004, Rev 02}

\section{Mitigation Feature}

Scenario Number:

U15089

Mitigation Number:

Scenario:

Personnel working on the mapping floor come into contact with operating muck conveyor system mechanical parts (e.g., conveyor belts, idlers, etc.)

Mitigation Feature: Stowage of tools and materials on the deck of the conveyor side of the mapping floor will be prohibited. The conveyor side of the three mapping floor cars and the two transition ramps will be kept clear of materials at all times.

Documentation:

Underground mapping of the ECRB JSA, 19jun98,page1.

Remarks:

JSA developed. Part of mapping floor training.

Stowage compartments are provided below the floor decks.

Mitigation type:
Design Feature
Safety Device
Warning Device
Procedure
Training

\section{Mitigation Tracking}

\section{Mitigation Implementation Status:}

Comments:
Not Implemented

implemented

\section{Appendix A}

Page UI5089.1 


\section{DI: BAB000000-01717-0200-0004, Rev 02}

\section{Mitigation Feature}

$\begin{array}{ll}\text { Scenario Number: } & \text { Mitigation Number: } \\ \text { Scenario: } & \begin{array}{l}\text { Personnel working on the mapping floor come into contact with operating muck conveyor } \\ \text { system mechanical parts (e.g., conveyor belts, idlers, etc.) }\end{array} \\ \text { Mitigation Feature: } & \begin{array}{l}\text { Install handrails equipped with small-opening mesh on the conveyor side of the mapping floor } \\ \text { cars to prevent personnel from inadvertently entering/reaching into the conveyor area. }\end{array} \\ \text { Documentation: } & \begin{array}{l}\text { Tortoise fencing is in place over handrails with holes in fencing to allow access to the E-stops. } \\ \text { Remarks: }\end{array} \\ \text { Mitigation type: } & \begin{array}{l}\text { Design Feature } \\ \text { Safety Device }\end{array} \\ \text { Warning Device } \\ \text { Procedure } \\ \text { Training }\end{array}$

\section{Mitigation Tracking}

\section{Mitigation Implementation Status:}

Comments:
Not Implemented

Implemented

\section{Appendix A}

Page UI5089.2 


\section{DI: BAB000000-01717-0200-0004, Rev 02}

\section{Mitigation Feature}

Scenario Number: $\quad$ U15089 Mitigation Number: 3

Scenario:

Personnel working on the mapping floor come into contact with operating muck conveyor system mechanical parts (e.g., conveyor belts, idlers, etc.)

Mitigation Feature:

The conveyor system shall be tagged out/locked out to prevent conveyor operation whenever mapping survey or scientific evaluation of the tunnel walls above or below the conveyor would expose persannel to the moving mechanical parts of the conveyor system. Mitigation requires coordination with TBM operations.

Documentation: Underground mapping of the ECRB JSA, 19jun98,page1.

Remarks:

JSA developed. Part of mapping floor training. Work on the conveyor side is limited to painting of rock fractures above the conveyor.

\begin{tabular}{l|l} 
Mitigation type: & $\bigcirc$ Design Feature \\
$\bigcirc$ Safety Device \\
$\bigcirc$ Warning Device \\
Procedure \\
Training
\end{tabular}

\section{Mitigation Tracking}

Mitigation Implementation Status:

Comments:
Not Implemented

Implemented

Appendix A

Page UI5089.3 


\section{DI: BAB000000-01717-0200-0004; Rev 02}

\section{Mitigation Feature}

Scenario Number:

U15089

Mitigation Number:

4

Scenario:

Personnel working on the mapping floor come into contact with operating muck conveyor system mechanical parts (e.g., conveyor belts, idlers, etc.)

Mitigation Feature: Personnel working on the mapping floor shall be trained to never enter the two foot wide section of the floor that is under the conveyor system unless the conveyor is locked/tagged out. A lack/tag shall be applied by the mapping crew supervisor in addition to any others.

Documentation: Underground mapping of the ECRB JSA, 19jun98, page1.

Remarks:

JSA developed. Part of mapping floor training.

Mitigation type:
Design Feature
Safety Device
Warning Device
$\bigcirc$ Procedure
Training

\section{Mitigation Tracking}

Mitigation

Implementation

Status:

Comments:
Not Implemented

implemented

\section{Appendix A}

Page UI5089.4 
DI: BAB000000-01717-0200-0004, Rev 02

\section{Mitigation Feature}

Scenario Number:

U15089

Mitigation Number:

6

Scenario:

Personnel working on the mapping floor come into contact with operating muck conveyor system mechanical parts (e.g., conveyor belts, idlers, etc.)

Mitigation Feature: The emergency stop cord for the conveyor system will be placed so it is accessible from both the upper mapping floor and the rail deck floor without exposing personnel to moving conveyor parts.

Documentation:

Remarks:

There are holes in the mesh fencing that allow access to the E-stop cords.

Mitigation type:
Design Feature
Safety Device
Warning Device
Procedure
Training

\section{Mitigation Tracking}

\section{Mitigation Implementation Status:}

Not Implemented

implemented

\section{Comments:}

\section{Appendix A \\ Page UI5089.6}




\section{Approved}

Scenario Number:

Revision Number:

Revision Date:

\section{Scenario:}

Cause, Failure, or Hazardous Event:

Risk Assignment Before Considering Mitigations:

Risk Designation After Applying Mitigations:

Related Scenarios:

Prevention Mitigation Documentation:

\begin{tabular}{|l|}
\hline U15091 \\
\hline 02 \\
\hline $12 / 17 / 98$ \\
\hline
\end{tabular}

SSA: East-West Drift

Location: Mapping Floor

Doors positioned to provide clearance for train passage fall into path of the train due to vibration of the mapping floor or inadvertent personnel acts that dislodge doors from vertical pasition.

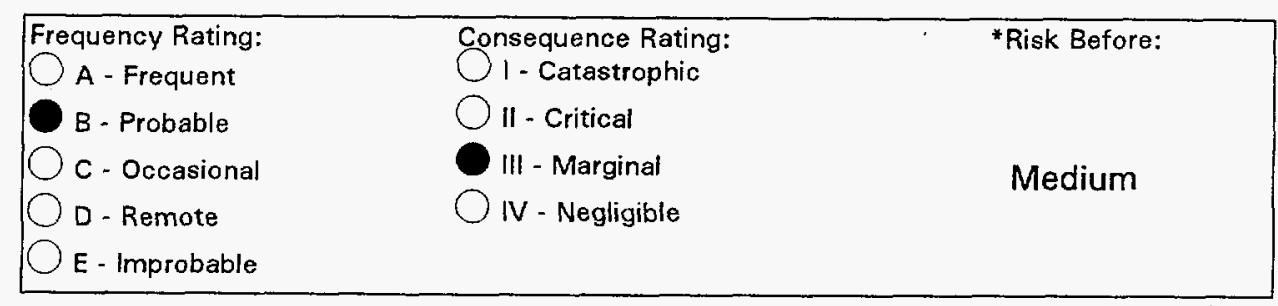

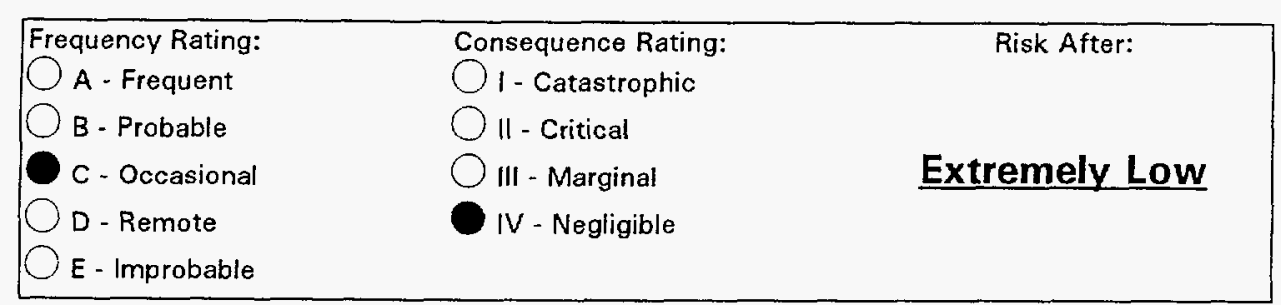

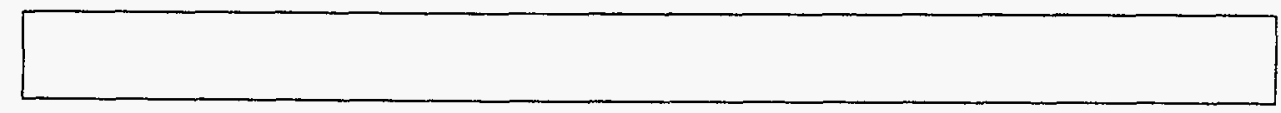

Underground Mapping of the ECRB JSA, 19jun98, page1.

\section{Appendix A}

Page UI5091 


\section{DI: BAB000000-01717-0200-0004, Rev 02}

\section{Mitigation Feature}

Scenario Number: Ui5091 Mitigation Number:

Scenario: $\quad$ Mapping floor door(s) fall into path of train or against moving train or cars.

Mitigation Feature: Install automatic latches to hold each door in the open vertical position.

Documentation:

Remarks:

Pins installed.

Mitigation type:

Design Feature

Safety Device

Warn!ng Device

Procrogure

Tram:

\section{Mitigation Tracking}

Mitigation

Implementation

Status:

Comments:
Not Implemented

Implemented
Appendix A

Page UI5091.1 
DI: BAB000000-01717-0200-0004, Rev 02

\section{Mitigation Feature}

Scenario Number:

U15091

Mitigation Number:

2

Scenario:

Mapping floor door(s) fall into path of train or against moving train or cars.

Mitigation Feature:

Equipment and/or materials will not be leaned or braced against the raised vertical mapping

floor doors.

Documentation: Underground mapping of the ECRB JSA, 19jun98,page1.

Remarks:

JSA developed. Covered in mapping floor JSA training.

Mitigation type:
Design Feature
Safety Device
Warning Device
Procedure
Training

\section{Mitigation Tracking}

\section{Mitigation \\ Implementation \\ Status:}

Comments:
Not Implemented

Implemented 
DI: BAB000000-01717-0200-0004, Rev 02

\section{Mitigation Feature}

Scenario Number:

U15091

Mitigation Number:

3

Scenario:

Mapping floor door(s) fall into path of train or against moving train or cars.

Mitigation Feature: Personnel will not brace themselves or lean against the raised vertical mapping floor doors.

Documentation: Underground mapping of the ECRB JSA, 19jun98,page1.

Remarks:

JSA developed. Covered in mapping floor JSA training.

Mitigation type:
Design Feature
Safety Device
Warning Device
Procedure
Training

\section{Mitigation Tracking}

Mitigation

Implementation

Status:

Not Implemented

Implemented

Comments:

\section{Appendix A}

Page UI5091.3 


\section{Scenario Description and Analysis}

\section{Approved}

Scenario Number:

Revision Number:

Revision Date:

Scenario:

Cause, Failure, or Hazardous Event:

Risk Assignment Before Considering Mitigations:

\section{After}

Risk Designation

Applying

Mitigations:

Related Scenarios:

Prevention

Mitigation

Documentation:

\begin{tabular}{|l|}
\hline$U 15092$ \\
\hline 02 \\
\hline $12 / 17 / 98$ \\
\hline
\end{tabular}

SSA: East-West Drift

Location: Mapping Floor

Mapping floor equipment protrudes into the train envelope. Mapping floor doors are left in horizontal position and are not positioned vertically for train transit. Train operator fails to see horizontal floor panel obstructions.

\begin{tabular}{|c|c|c|}
\hline Frequency Rating: & Consequence Rating: & * Risk Before: \\
\hline A - Frequent & I - Catastrophic & \\
\hline B - Probable & II - Critical & \\
\hline O-Occasional & II - Marginal & Medium \\
\hline OD-Remote & IV - Negligible & \\
\hline E-Improbable & & \\
\hline
\end{tabular}

\begin{tabular}{|lll|}
\hline Frequency Rating: & Consequence Rating: & Risk After: \\
A - Frequent & OI-Catastrophic & \\
B - Probable & $\bigcirc$ & \\
C - Occasional & $O$ - Critical & Extremely Low \\
D-Remote & - Marginal & \\
E- Improbable & IV - Negligible & \\
\hline
\end{tabular}

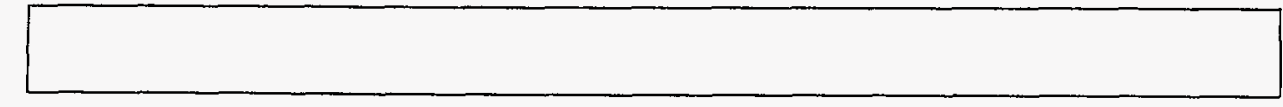

Underground Mapping of the ECRB, 19jun98, page2.

\section{Appendix A \\ Page U15092}




\section{DI: BAB000000-01717-0200-0004; Rev 02}

\section{Mitigation Feature}

Scenario Number:

U15092 Mitigation Number:

1

Scenario:

Train strikes equipment or mapping floor doors while transiting the mapping floor.

Mitigation Feature:

The train operator shall always stop the train before entering the mapping floor. The train brakeman shall walk ahead of the train across the mapping floor to determine that the track is clear. He will signal the train to stop is any obstacles (e.g., equipment, hoses or cables) are found in the train path.

Documentation:

Remarks:

Mitigation type:
Design Feature
Safety Device
Warning Device

Procedure

Training

\section{Mitigation Tracking}

Mitigation

Implementation

Status:

Comments:
Not Implemented

implemented

\section{Appendix A}

Page UI5092.1 


\section{DI: BAB000000-01717-0200-0004, Rev 02}

\section{Mitigation Feature}

$\begin{array}{ll}\text { Scenario Number: } & \text { Ul5092 } \\ \text { Scenario: } & \text { Mitigation Number: } \\ \text { Mitigation Feature: } & \begin{array}{l}\text { Install high-visibility reflective materials (e.g., tape or paint) on both the heading and portal } \\ \text { edges of all the mapping floor door support frames so that the train operator can clearly see } \\ \text { any doors that are left in the down position. }\end{array} \\ \text { Documentation: } & \\ \text { Remarks: } & \\ \text { Mitigation type: } & \text { Design Feature } \\ \text { Safety Device } \\ \text { Warning Device } \\ \text { Procedure }\end{array}$

\section{Mitigation Tracking}

Mitigation Implementation Status:

\section{Comments:}

Not Implemented

Implemented

\section{Appendix A}

Page UI5092.2 


\section{DI: BAB000000-01717-0200-0004, Rev 02}

\section{Mitigation Feature}

Scenario Number: $\quad$ U15092 Mitigation Number: 3

Scenario:

Train strikes equipment or mapping floor doors while transiting the mapping floor.

Mitigation Feature: The mapping floor doors shall be raised and locked in a vertical position prior to train passage.

Documentation:

Remarks:

JSA developed. Covered in mapping floor training.

Mitigation type:
Design Feature
Safety Device
Warning Device
Procedure
Training

\section{Mitigation Tracking}

Mitigation

Implementation

Status:

Comments:
Not Implemented

implemented 


\section{DI: BABO00000-01717-0200-0004; Rev 02}

Scenario Number:
Scenario:
Mitigation Feature: $\begin{array}{ll}\text { The mapping floor work supervisor will insure that all doors are properly secured and no } \\ \text { equipment protrudes or overhangs into the transit space. He will then signal (e.g., hand and } \\ \text { arm) the train operator that the mapping floor is prepared for passage of the train. }\end{array}$
Documentation:
Rnderground Mapping of the ECRB, 19jun98, page 2.
Remarks:
Mitigation type:
JSA developed. Covered in mapping floor JSA training.
$\begin{aligned} & \text { Design Feature } \\ & \text { Safety Device }\end{aligned}$
$\begin{aligned} & \text { Warning Device } \\ & \text { Procedure }\end{aligned}$
Training

\section{Mitigation Tracking}

Mitigation

Implementation

Status:

Comments:
Not Implemented

Impiemented

Appendix A

Page UI5092.4 


\section{DI: BAB000000-01717-0200-0004, Rev 02}

\section{Mitigation Feature}

Scenario Number: U15092 Mitigation Number: 5

Scenario:

Train strikes equipment or mapping floor doors while transiting the mapping floor.

Mitigation Feature: The train operator shall limit the speed of the train across the mapping floor to a very slaw speed.

Documentation: Underground Mapping of the ECRB, 19jun98, page 2.

Remarks:

JSA developed. Part of mapping floor training.

Mitigation type:
Design Feature
Safety Device
Warning Device
Procedure
Training

\section{Mitigation Tracking}

Mitigation

Implementation

Status:

Comments:
Not Implemented

Implemented

\section{Appendix A}

Page UI5092.5 


\section{DI: BAB000000-01717-0200-0004, Rev 02}

\section{Mitigation Feature}

Scenario Number: 415092

Scenario:

Train strikes equipment or mapping floor doors while transiting the mapping floor.

Mitigation Feature: Derailers will be installed approximately 60 feet in front and behind the mapping floor to insure that the train can not reach the mapping floor until the area is prepared for train passage and the derailers have been removed.

Documentation:

Remarks:

Mitigation type:
Design Feature
Safety Device
Warning Device
Procedure
Training

Mitigation Tracking

\section{Mitigation \\ Implementation \\ Status:}

Comments: 


\section{Scenario Description and Analysis}

\section{Approved}

Scenario Number:

Revision Number:

Revision Date:

Scenario:

Cause, Failure, or Hazardous Event:

Risk Assignment Before Considering Mitigations:

\begin{tabular}{|l|}
\hline$U 15093$ \\
\hline 02 \\
\hline $12 / 17 / 98$ \\
\hline
\end{tabular}

Train strikes personnel while transiting the mapping floor.

Personnel inadvertently enter the train envelope or fall against moving train/cars in transit of the floor.

\begin{tabular}{|lll|}
\hline Frequency Rating: & Consequence Rating: & *Risk Before: \\
A - Frequent & I- Catastrophic & \\
B - Probable & II - Critical & Medium \\
C - Occasional & III - Marginal & \\
D - Remote & IV - Negligible & \\
E - Improbable & & \\
\hline
\end{tabular}

Risk Designation
After
Applying
Mitigations:

Frequency Rating:

Consequence Rating:

Risk After:

A - Frequent

I - Catastrophic

B - Probable

II - Critical

C - Occasional

OIII - Marginal

Low

D - Remote

OIV - Negligible

E - Improbable

Related Scenarios:

Prevention

Mitigation

Documentation:
Underground Mapping of the ECRB, 19jun98, page2. 


\section{DI: BAB000000-01717-0200-0004; Rev 02}

\section{Mitigation Feature}

Scenario Number: $\quad$ U15093 Mitigation Number: 1

\section{Scenario: $\quad$ Train strikes personnel while transiting the mapping floor.}

Mitigation Feature: The train operator shall always stop the train before entering the mapping floor. The brakeman will precede the train and ensure that the train envelop is clear of personnel prior to the train entering the floor.

Documentation:

Remarks:

Mitigation type:
Design Feature
Safety Device
Warning Device
Procedure
Training

\section{Mitigation Tracking}

Mitigation

Implementation

Status:

Comments:
Not Implemented

Implemented

Appendix A

Page UI5093.1 


\section{DI: BAB000000-01717-0200-0004, Rev 02}

\section{Mitigation Feature}

$\begin{array}{ll}\text { Scenario Number: } & \text { Ul5093 } \\ \text { Scenario: } & \text { Mitigation Number: } \\ \text { Mitigation Feature: } & \begin{array}{l}\text { All personnel working on the mapping floor shall wear high-visibility reflective material/tape on } \\ \text { hard hats and clothing (e.g., vests, etc.) so that their location(s) are clearly visible to the train } \\ \text { operator. }\end{array} \\ \text { Documentation: } & \text { Underground Mapping in the ECRB, 19jun98, page 2. } \\ \text { Remarks: } & \begin{array}{l}\text { JSA developed. Part of mapping floor training. (The high-visibility reflective material/tape } \\ \text { requirement applies to all personnel in the ECRB). }\end{array} \\ \text { Mitigation type: } & \begin{array}{l}\text { Design Feature } \\ \text { Safety Device }\end{array} \\ \text { Warning Device } \\ \text { Procedure } \\ \text { Training }\end{array}$

\section{Mitigation Tracking}

Mitigation Implementation Status:

\section{Comments:}

Not Implemented

$O$ Implemented 


\section{DI: BAB000000-01717-0200-0004, Rev 02}

\section{Mitigation Feature}

Scenario Number:

U15093

Mitigation Number:

Scenario:

Train strikes personnel while transiting the mapping floor.

Mitigation Feature:

Mapping floor personnel shall cease scientific evaluation and survey work during preparation and passage of the train over the mapping floor. Personnel shall stand in place on the two foot wide utility side of the mapping floor. Personnel will not move about on the mapping floor during train passage.

Documentation: Underground Mapping of the ECRB, 19jun98, page 2.

Remarks: $\quad$ JSA developed. Part of mapping floor training.

Mitigation type: $\bigcirc$ Design Feature

Safety Device

Warning Device

Procedure

Training

\section{Mitigation Tracking}

\section{Mitigation Implementation Status:}

\section{Comments:}

Not Impiemented

Implemented

\section{Appendix A}

Page UI5093.3 


\section{DI: BAB000000-01717-0200-0004, Rev 02}

\section{Mitigation Feature}

Scenario Number: $\quad$ Ul5093 Mitigation Number: 4

Scenario:

Train strikes personnel while transiting the mapping floor.

Mitigation Feature:

The mapping floor supervisor or designee shall signal (e.g., hand and arm) the train operator/brakeman when all personnel are prepared for passage of the train. Any personnel may signal the train operator to stop if danger or a potential problem is perceived while the train is transiting the floor.

Documentation:

Underground Mapping of the ECRB, 19jun98, page 2.

Remarks:

JSA developed. Part of mapping floor training.

Mitigation type:

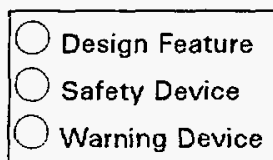

Procedure

Training

\section{Mitigation Tracking}

Mitigation

Implementation

Status:

Comments:
Not Impiemented

Implemented

\section{Appendix A}

Page UI5093.4 


\section{DI: BAB000000-01717-0200-0004, Rev 02}

\begin{tabular}{|c|c|}
\hline \multicolumn{2}{|r|}{ Mitigation Feature } \\
\hline Scenario Number: & Mitigation Number: \\
\hline Scenario: & Train strikes personnel while transiting the mapping floor. \\
\hline Aitigation Feature: & $\begin{array}{l}\text { The train operator shall blow the horn prior to entering the mapping floor and limit the speed } \\
\text { of the train across the mapping floor to a very slow speed. }\end{array}$ \\
\hline Jocumentation: & Underground Mapping in the ECRB, 19jun98, page 2. \\
\hline Remarks: & JSA developed. Part of mapping floor training. \\
\hline Mitigation type: & $\begin{array}{l}\text { Design Feature } \\
\text { Safety Device } \\
\text { warning Device } \\
\text { irocedure } \\
\text { iraining }\end{array}$ \\
\hline
\end{tabular}

\section{Mitigation Tracking}

Mitigation Implementation Status:

Comments:
Not Implemented

Implemented

\section{Appendix A \\ Page U15093.5}


DI: $B A B 000000-01717-0200-0004 ; \operatorname{Rev} 02$

\section{Mitigation Feature}

Scenario Number: U15093 Mitigation Number:

Scenario: $\quad$ Train strikes personnel while transiting the mapping floor.

Mitigation Feature: All personnel (e.g., scientific, engineering, maintenance, etc.) who will work on the mapping floor will receive a safety briefing to emphasize the personnel hazards of working on the mapping floor and detailed instructions regarding procedures for passage of the train across the mapping floor.

Documentation:

Remarks:

JSA developed. Covered in mapping floor JSA training.

Mitigation type:
Design Feature
Safety Device
Warning Device
Procedure
Training

\section{Mitigation Tracking}

Mitigation

Implementation

Status:

Comments:
Not Implemented

O Implemented

Appendix A

Page UI5093.6 
DI: BAB000000-01717-0200-0004, Rev 02

\section{Mitigation Feature}

Scenario Number:

U15093 Mitigation Number:

Scenario:

Train strikes personnel while transiting the mapping floor.

Mitigation Feature: Personnel shall be trained to never attempt to enter a moving train while it is transiting the mapping floor. Same rule applies to personnel on a moving train not to attempt exiting onto the mapping floor.

Documentation: Underground Mapping of the ECRB, 19jun98, page 2.

Remarks:

JSA developed. Covered in mapping floor JSA training.

Mitigation type:
Design Feature
Safety Device
Warning Device
Procedure
Training

\section{Mitigation Tracking}

Mitigation

Implementation

Status:

Comments:
Not Implemented

O Implemented 


\section{DI: BAB000000-01717-0200-0004; Rev 02}

\section{Mitigation Feature}

Scenario Number: UI5093 Mitigation Number:

8

Scenario:

Train strikes personnel while transiting the mapping floor.

Mitigation Feature: The train brakeman precedes the train across the mapping floor to determine that the track is clear. He will signal the train to proceed only after all obstacles (e.g., equipment, hoses or cables) and personnel are clear of the train path.

Documentation: Undergrend Mapping of the ECRB, 19 jun98, page 2.

Remarks:

JSA de:

Mitigation type:
Desitin -aature
Saferv Yavice
Warring Device
Procedure
Trainiry

\section{Mitigation Tracking}

Mitigation

Implementation

Status:

Comments:
Not Implemented

Implemented

Appendix A

Page UI5093.8 


\section{Approved}

Scenario Number:

Revision Number:

Revision Date:

\section{Scenario:}

Cause, Failure, or Hazardous Event:

Risk Assignment Before Considering Mitigations:

Prevention

Mitigation

Documentation:

\begin{tabular}{l} 
Risk Designation \\
After \\
\hline Applying \\
Mitigations:
\end{tabular}

\begin{tabular}{|l|}
\hline U15094 \\
\hline 02 \\
\hline $12 / 17 / 98$ \\
\hline
\end{tabular}

\author{
SSA: East-West Drift \\ Location: Mapping Floor
}

Minor personnel injuries due to fall/trip/slip on the mapping floor.

On the mapping floor car with doors there are uneven floor surface(s), open gaps lapprox. 1.5 inches) between closed doors. There is an 16-inch drop from the center mapping floor to the rail deck well on adjoining mapping cars. Debris on all mapping floor cars (e.g., loose tools, electrical cables, personal items (e.g., jackets, lunch boxes, etc.).

\begin{tabular}{|lll|}
\hline Frequency Rating: & Consequence Rating: & *Risk Before: \\
A - Frequent & II-Catastrophic & \\
B - Probable & III-Critical & Medium \\
C - Occasional & III - Marginal & \\
D-Remote & IV - Negligible & \\
E- Improbable & & \\
\hline
\end{tabular}

\begin{tabular}{|lll|}
\hline Frequency Rating: & Consequence Rating: & Risk After: \\
A - Frequent & $O_{1-\text { Catastrophic }}$ & \\
B - Probable & $O_{I I-\text { Critical }}$ & \\
C - Occasional & $O_{I I I}$ - Marginal & Low \\
D- Remote & $O_{\mathrm{N} \text { - Negligible }}$ & \\
E- Improbable & & \\
\hline
\end{tabular}

\section{UI5047, U15069}

Underground Mapping of the ECRB, 19jun98, page3.

\section{Appendix A \\ Page UI5094}




\section{DI: BAB000000-01717-0200-0004; Rev 02}

\section{Mitigation Feature}

$\begin{array}{ll}\text { Scenario Number: } & \text { Ul5094 } \\ \text { Scenario: } & \text { Minor personnel injuries due to fall/trip/slip on the mapping floor. } \\ \text { Mitigation Feature: } & \text { To prevent falls/trips/slips, small items to the extent practical shall be tethered to prevent their } \\ \text { cluttering the mapping floor. } & \\ \text { Documentation: } & \text { Underground Mapping of the ECRB, 19jun98, page 3. } \\ \text { Remarks: } & \text { JSA developed. Covered in mapping floor JSA training. } \\ \text { Mitigation type: } & \text { Design Feature } \\ \text { Safety Device } \\ \text { Warning Device } \\ \text { Procedure } \\ \text { Training }\end{array}$

\section{Mitigation Tracking}

Mitigation

Implementation

Status:

Comments:
Not Implemented

Implemented 


\section{DI: BAB000000-01717-0200-0004, Rev 02}

\section{Mitigation Feature}

Scenario Number:

$$
015094
$$

Mitigation Number

2

Scenario:

Minor personnel injuries due to fall/trip/slip on the mapping floor.

Mitigation Feature: Electrical cables not in use will be stored in the mapping floor storage compartments and not left on the mapping floor surface or rail deck.

Documentation:

Underground Mapping of the ECRB, 19jun98, page 3

Remarks:

JSA developed. Part of mapping floor training.

Mitigation type:
Design Feature
Safety Device
Warning Device
Procedure
Training

\section{Mitigation Tracking}

\section{Mitigation \\ Implementation \\ Status:}

\section{Comments:}

\section{Appendix A \\ Page UI5094.2}


DI: BAB000000-01717-0200-0004, Rev 02

\section{Mitigation Feature}

Scenario Number: $\quad$ UI5094 Mitigation Number: 3

\section{Scenario: $\quad$ Minor personnel injuries due to fall/trip/slip on the mapping floor.}

Mitigation Feature: Temporary task lighting on stands or pedestals shall be provided to illuminate all mapping floor work areas where tunnel lighting is inadequate for scientific/survey tasks.

Documentation:

Remarks:

Mitigation type: $\bigcirc$ Design Feature

Safety Device

Warning Device

Procedure

Training

\section{Mitigation Tracking}

Mitigation

Implementation

Status:

Comments:
Not Implemented

Implemented

Appendix A

Page UI5094.3 


\section{DI: BAB000000-01717-0200-0004, Rev 02}

\section{Mitigation Feature}

Scenario Number: UI5094 Mitigation Number: 4

Scenario: $\quad$ Minor personnel injuries due to fall/trip/slip on the mapping floor.

Mitigatior: Feature: Personnel working on the mapping floor will be provided with a designated storage location for personnel items (e.g., jackets, lunch boxes, etc.) so they do not clutter the mapping floor.

Documentation:

Remarks:

Storage areas have been added on the conveyor side with access from the side wall of the rail deck floor.

Mitigation type:
Design Feature
Safety Device
Warning Device
Procedure
Training

\section{Mitigation Tracking}

\section{Mitigation Implementation Status:}

\section{Comments:}

Not Implemented

0 implemented

Appendix A

Page UI5094.4 


\section{DI: BAB000000-01717-0200-0004, Rev 02}

\section{Mitigation Feature}

$\begin{array}{ll}\text { Scenario Number: } & \text { Mis094 } \\ \text { Scenario: } & \text { Minor personnel injuries due to fall/trip/slip on the mapping floor. } \\ \text { Mitigation Feature: } & \begin{array}{l}\text { Provide safety training and orientation to personnel working on the mapping floor with } \\ \text { information regarding the floor conditions (e.g., uneven areas, gaps, } 16 \text { inch drop between the } \\ \text { floor and the rail deck, etc.) }\end{array} \\ \text { Documentation: } & \begin{array}{l}\text { Remarks: } \\ \text { Remation }\end{array} \\ \text { Mitigation type: } & \begin{array}{l}\text { Design Feature } \\ \text { Safety Device } \\ \text { Warning Device } \\ \text { Procedure } \\ \text { Training }\end{array}\end{array}$

\section{Mitigation Tracking}

Mitigation

Implementation

Not Implemented

Status:

Implemented

Comments:

\section{Appendix A}

Page UI5094.5 
DI: BAB000000-01717-0200-0004; Rev 02

\section{Mitigation Feature}

Scenario Number: $415094 \quad$ Mitigation Number: 6

Scenario: $\quad$ Minor personnel injuries due to fall/trip/slip on the mapping floor.

Mitigation Feature: Frequently perform "housekeeping" tasks to keep the working floor area clear. Specifically accomplish the housekeeping chores prior to opening the doors for train passage.

Documentation: Underground Mapping of the ECRB, 19jun98, page3.

Remarks: $\quad$ JSA developed. Covered in mapping floor JSA training.

Mitigation type: $\bigcirc$ Design Feature

Safety Device

Warning Device

Procedure

Training

\section{Mitigation Tracking}

Mitigation Implementation Status:

Comments:

\section{Not Implemented}

Implemented 


\section{DI: ВAB000000-01717-0200-0004, Rev 02}

\section{Scenario Description and Analysis}

\section{Approved}

Scenario Number:

Revision Number:

Revision Date:

\begin{tabular}{|l|}
\hline 015096 \\
\hline 02 \\
\hline $12 / 17 / 98$ \\
\hline
\end{tabular}

SSA: East-West Drift

Location: Mapping Floor

\section{Scenario:}

Cause, Failure, or Hazardous Event:

Personnel injury due to crawling or becoming stuck under the Transition Ramp and/or Mapping Floor (either raised or lowered) to retrieve items.

Personnel attempt to recover dropped items from beneath the Transition Ramp or Mapping Floor.

Risk Assignment

Before Considering

Mitigations:

\begin{tabular}{|lll|}
\hline Frequency Rating: & Consequence Rating: & *Risk Before: \\
A - Frequent & I-Catastrophic & \\
B - Probable & II - Critical & Medium \\
C - Occasional & III - Marg!nal & \\
D-Remote & IV - Ne - igible & \\
E - Improbable & & \\
\hline
\end{tabular}

Risk Designation

After

Applying

Mitigations:

Frequency Rating:
A - Frequent
B - Probable
C - Occasional
D - Remote
E - improbable

Related Scenarios:

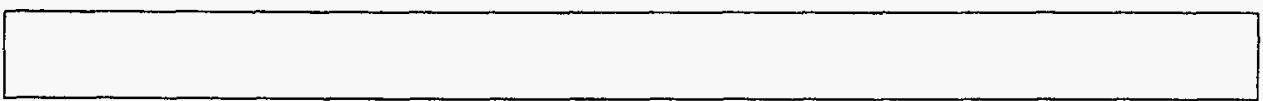

Prevention

Underground Mapping of the ECRB, 19jun98, page3.

Mitigation

Documentation:

\section{Appendix A}

Page UI5096 


\section{DI: BAB000000-01717-0200-0004; Rev 02}

\section{Mitigation Feature}

Scenario Number:

U15096

Mitigation Number:

1

Scenario:

Personnel injury due to crawling or becoming stuck under the Transition Ramp and/or Mapping Floor (either raised or lowered) to retrieve items.

Mitigation Feature:

Personnel shall be prohibited from crawling under any of the mapping floor cars for any

reason. Dropped items shall be retrieved when the mapping floor is moved.

Documentation: Underground Mapping of the ECRB, 19jun98, page3.

Remarks: $\quad$ JSA developed. Part of mapping floor training.

Mitigation type: $\quad$\begin{tabular}{|l}
$\bigcirc$ Design Feature \\
$\bigcirc$ Safety Device \\
$\bigcirc$ Warning Device \\
Procedure \\
$\bigcirc$ Training
\end{tabular}

\section{Mitigation Tracking}

Mitigation

Implementation

Status:

Not Implemented

Implemented

Comments:

Appendix A

Page UI5096.1 


\section{Scenario Description and Analysis}

\section{Approved}

Scenario Number:

Revision Number:

Revision Date:

\section{Scenario:}

Cause, Failure, or Hazardous Event:

Risk Assignment Before Considering Mitigations:

\begin{tabular}{|l|}
\hline UI5097 \\
\hline 02 \\
\hline $12 / 17 / 98$ \\
\hline
\end{tabular}
supports.

\author{
SSA: East-West Drift \\ Location: Mapping Floor
}

Personnel injury (e.g., pinch, amputation, crush) from opening/closing the mapping floor doors or the access panels to the pressurized air system valves (located in the rail decks) of the transition ramp/mapping floor cars.

Personnel hands/fingers extend into mapping floor pinch points while opening/closing the doors. Personnel lowering access panels catch fingers between the panel and the floor well

\begin{tabular}{|lll|}
\hline Frequency Rating: & Consequence Rating: & *Risk Before: \\
A - Frequent & $O_{\text {I - Catastrophic }}$ & \\
B - Probable & II - Critical & Medium \\
C - Occasional & III- Marginal & \\
D-Remote & IV - Negligible & \\
E- Improbable & & \\
\hline
\end{tabular}

Risk Designation

After

Applying

Mitigations:

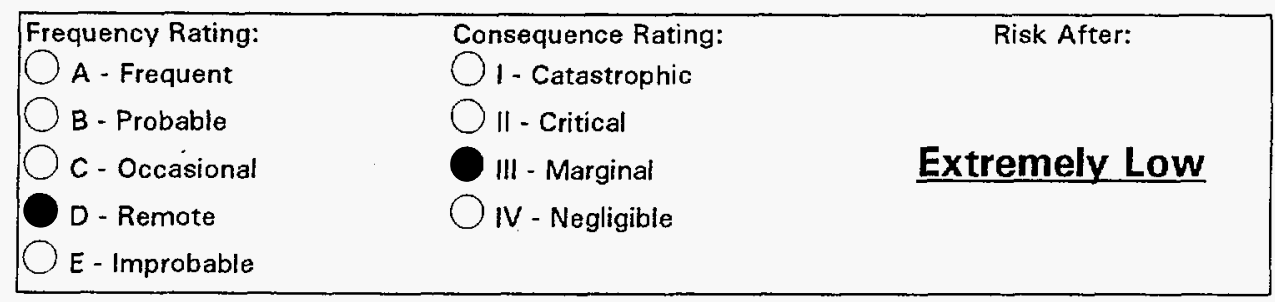

Related Scenarios:

Prevention

Mitigation

Documentation: 
DI: BAB000000-01717-0200-0004, Rev 02

\section{Mitigation Feature}

Scenario Number: $\quad$ U15097 Mitigation Number: 1

Scenario: $\quad$ Personnel injury (e.g., pinch, amputation, crush) from opening/closing the mapping floor doars or the access panels to the pressurized air system valves (located in the rail decks) of the transition ramp/mapping floor cars.

Mitigation Feature: Personnel will open/close the mapping floor doors holding the door sides only while standing in the rail deck well. Personnel will keep hands and fingers clear of the hinge edge and center edges where doors join.

Documentation: Underground Mapping of the ECRB, 19 jun98, page 4.

Remarks: $\quad$ JSA developed. Covered in mapping floor JSA training.

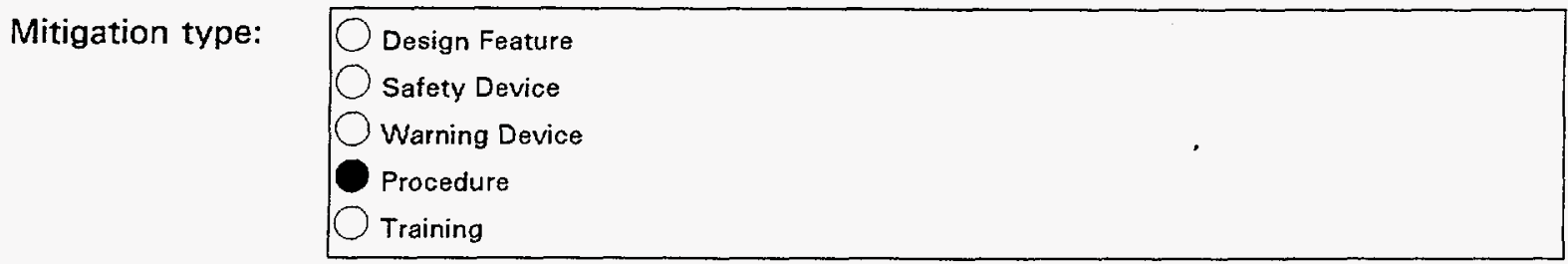

\section{Mitigation Tracking}

Mitigation

Implementation

Status:

Comments:
Not Implemented

Implemented 
DI: BAB000000-01717-0200-0004, Rev 02

\section{Mitigation Feature}

Scenario Number: $\quad$ U15097 Mitigation Number: 2

Scenario: $\quad$ Personnel injury (e.g., pinch, amputation, crush) from opening/closing the mapping floor doors or the access panels to the pressurized air system valves (located in the rail decks) of the transition ramp/mapping floor cars.

Mitigation Feature: $\sqrt{\text { Install fold down handles on the access panels or move the hand holes } 2 \text { to } 3 \text { inches away }}$ from the floor supports.

Documentation:

Remarks:

Mitigation type:

Design Feature

Safety Device

Warning Device

$\bigcirc$ Procedure

Training

\section{Mitigation Tracking}

Mitigation

Implementation

Status:

Comments:
Not Implemented

Implemented

\section{Appendix A}

Page UI5097.2 


\section{Scenario Description and Analysis}

\section{Approved}

Scenario Number:

Revision Number:

Revision Date:

\begin{tabular}{|l|}
\hline U15098 \\
\hline 02 \\
\hline $12 / 17 / 98$ \\
\hline
\end{tabular}

SSA: East-West Drift

Location: Mapping Floor

\section{Scenario:}

If the air winches (tuggers) are ever used to move the mapping floor (in raised configuration) along the tracks to a new location and a cable fails or slips resulting in personnel injury.

Cause, Failure, or Hazardous Event:

Cable fails or slips under high tension.

Risk Assignment Before Considering Mitigations:
Frequency Rating:
$\bigcirc$ A - Frequent
B - Probable
C - Occasional
D-Remote
E - Improbable

$\begin{array}{ll}\text { Consequence Rating: } & \text { "Risk Before: } \\ \text { I- Catastrophic } & \\ \text { II - Critical } & \\ \text { III - Marginal } & \text { Medium } \\ \text { IV - Negligible } & \end{array}$

Risk Designation

After

Applying

Mitigations:

\begin{tabular}{|lll|}
\hline Frequency Rating: & Consequence Rating: & Risk After: \\
A - Frequent & OI-Catastrophic & \\
B - Probable & OII - Critical & Extremely Low \\
C - Occasional & $O I I$ - Marginal & \\
D - Remote & O - Negligible & \\
E- Improbable & & \\
\hline
\end{tabular}

Related Scenarios:

Prevention

Mitigation

Documentation: 


\section{DI: BAB000000-01717-0200-0004, Rev 02}

\section{Mitigation Feature}

Scenario Number: $\quad$ U15098 Mitigation Number: 1

Scenario:

If the air winches (tuggers) are ever used to move the mapping floor (in raised configuration) along the tracks to a new location and a cable fails or slips resulting in personnel injury.

Mitigation Feature: Install guards on the air winches at both ends of the mapping floor to protect the operator(s) from cable failures or slips.

Documentation:

Remarks:

Mitigation type: $\bigcirc$ Design Feature

Safety Device

Warning Device

$\bigcirc$ procedure

O Training

Mitigation Tracking

Mitigation

Implementation

Status:

Comments:
Not Implemented

Implemented

Appendix A

Page UI5098.1 


\section{Scenario Description and Analysis}

\section{Approved}

Scenario Number:

Revision Number:

Revision Date:

\begin{tabular}{|l|}
\hline U15099 \\
\hline 02 \\
\hline $12 / 17 / 98$ \\
\hline
\end{tabular}

SSA: East-West Drift

\section{Scenario:}

Tow cable slips or breaks and the train operator loses positive control of the raised/moving mapping floor.

Cause, Failure, or Hazardous Event:

Tow cable slips or breaks while the mapping cars are elevated/moving and control of the mapping floor is lost.

Risk Assignment Before Considering Mitigations:

\begin{tabular}{|lll|}
\hline Frequency Rating: & Consequence Rating: & *Risk Before: \\
O - Frequent & $O_{\text {II - Catastrophic }}$ & \\
B - Probable & - Critical & Medium \\
C - Occasional & OIV - Marginal & \\
D-Remote & & \\
E- Improbable & & \\
\hline
\end{tabular}

Risk Designation

After

Applying Frequency Rating: Consequence Rating: Risk After:

Mitigations:

A - Frequent

1 - Catastrophic

B - Probable

II-Critical

C - Occasional

OIII - Marginal

Low

D - Remote

OIV - Negligible

E - Improbable

Related Scenarios:

Prevention

Mitigation

Documentation: 
DI: BAB000000-01717-0200-0004, Rev 02

\section{Mitigation Feature}

Scenario Number:

U15099 Mitigation Number:

1

Scenario:

Tow cable slips or breaks and the train operator loses positive control of the raised/moving mapping floor.

Mitigation Feature:

During movement of the mapping floor, a mapping floor air system operator will ride on the transition ramp above the air system controls (e.g., used to elevate the mapping floor) and if necessary will depressurize the air system in order to stop any uncontrolled movement of the mapping floor

Documentation:

Remarks:

Mitigation type:
Design Feature
Safety Device
Warning Device
Procedure
Training

Mitigation Tracking

\section{Mitigation \\ Implementation \\ Status:}

Comments:
Not Implemented

Implemented

Appendix A

Page UI5099.1 
DI: BAB000000-01717-0200-0004, Rev 02

\section{Mitigation Feature}

Scenario Number: U15099 Mitigation Number:

Scenario: $\quad$ Tow cable slips or breaks and the train operator loses positive control of the raised/moving mapping floor.

Mitigation Feature: The speed of the locomotive shall be very siow when towing the mapping floor

Documentation:

Remarks:

Mitigation type:
Design Feature
Safety Device
$\bigcirc$ Warning Device
Procedure
Training

\section{Mitigation Tracking}

\section{Mitigation Implementation Status:}

Comments:
Not Implemented

Implemented

Appendix A

Page UI5099.2 


\section{DI: BAB000000-01717-0200-0004, Rev 02}

\section{Mitigation Feature}

$\begin{array}{ll}\text { Scenario Number: } & \text { Ui5099 } \\ \text { Scenario: } & \begin{array}{l}\text { Tow cable slips or breaks and the train operator loses positive control of the raised/moving } \\ \text { mapping floor. }\end{array} \\ \text { Mitigation Feature: } & \begin{array}{l}\text { The locomotive will always tow the mapping floor. The locomotive will never push the } \\ \text { mapping floor. }\end{array} \\ \text { Documentation: } & \\ \text { Remarks: } & \\ \text { Mitigation type: } & \text { Design Feature } \\ \text { Safety Device } \\ \text { Warning Device } \\ \text { Procedure } \\ \text { Training }\end{array}$

\section{Mitigation Tracking}

Mitigation

Implementation

Status:

Comments:
Not Implemented

Implemented

Appendix A

Page UI5099.3 


\section{Scenario Description and Analysis}

\section{Approved}

Scenario Number: Revision Number: Revision Date:

Scenario:

Cause, Failure, or Hazardous Event:

Risk Assignment Before Considering Mitigations:

\begin{tabular}{|l|}
\hline 015100 \\
\hline 02 \\
\hline $12 / 17 / 98$ \\
\hline
\end{tabular}

SSA

Location: Mapping Floor

Personnel are injured while riding on the elevated mapping cars during movement.

Personnel fall on mapping floor cars or are caught by passing conveyor or utility surfaces.

\begin{tabular}{|lll|}
\hline Frequency Rating: & Consequence Rating: & *Risk Before: \\
A - Frequent & $\bigcirc I-$ Catastrophic & \\
B - Probable & $\bigcirc I I-$ Critical & Medium \\
C - Occasional & $\bigcirc I I$ - Marginal & \\
D - Remote & $\bigcirc I V-$ Negligible & \\
E - Improbable & & \\
\hline
\end{tabular}

Risk Designation

After

Applying

Mitigations:

Frequency Rating:
A - Frequent
B - Probable
C - Occasional
D - Remote
E - Improbable

Consequence Rating:

I - Catastrophic

II - Critical

III - Marginal

OIV - Negligible

Risk After:

Extremely Low

Related Scenarios:

Prevention

Mitigation

Documentation:

Underground Mapping of the ECRB, 19jun98, page4.

\section{Appendix A \\ Page U15100}


DI: BAB000000-01717-0200-0004, Rev 02

\section{Mitigation Feature}

$\begin{array}{ll}\text { Scenario Number: } & \text { U15100 } \\ \text { Scenario: } & \text { Mersonnel are injured while riding on the elevated mapping cars during movement. } \\ \text { Mitigation Feature: } & \begin{array}{l}\text { During movement of the mapping floor all scientific/engineering personnel will be seated on } \\ \text { the utiities side of one of the mapping floor cars without doors with feet and legs extending } \\ \text { into the rail deck well. Personnel will not sit adjacent to the 3-inch gaps between cars. }\end{array} \\ \text { Documentation: } & \text { Underaround Mapping of the ECRB, 19jun98, page4. } \\ \text { Remarks: } & \text { JSA developed. Covered in mapping floor JSA training. } \\ \text { Mitigation type: } & \text { Design Feature } \\ \text { Safety Device } & \text { Warning Device } \\ \text { Procedure }\end{array}$

\section{Mitigation Tracking}

Mitigation Implementation

Status:

Comments:
Not Implemented

Impiemented

Appendix A

Page UI5100.1 


\section{Scenario Description and Analysis}

\section{Approved}

Scenario Number:

Revision Number:

Revision Date:

\section{Scenario:}

Cause, Failure, or Hazardous Event:

Risk Assignment Before Considering Mitigations:

\section{Risk Designation \\ After \\ Applying \\ Mitigations:}

Related Scenarios:

Prevention

Mitigation

Documentation:

\begin{tabular}{|l|}
\hline U15103 \\
\hline 02 \\
\hline $12 / 17 / 98$ \\
\hline
\end{tabular}

SSA: East-West Drift

Location: Mapping Floor

Personnel receive electrical shock(s) from defective electrical equipment that shorts/burns out or from severed electrical wire/connectors.

Electrical equipment shorts or burns out while in use on the mapping floor.

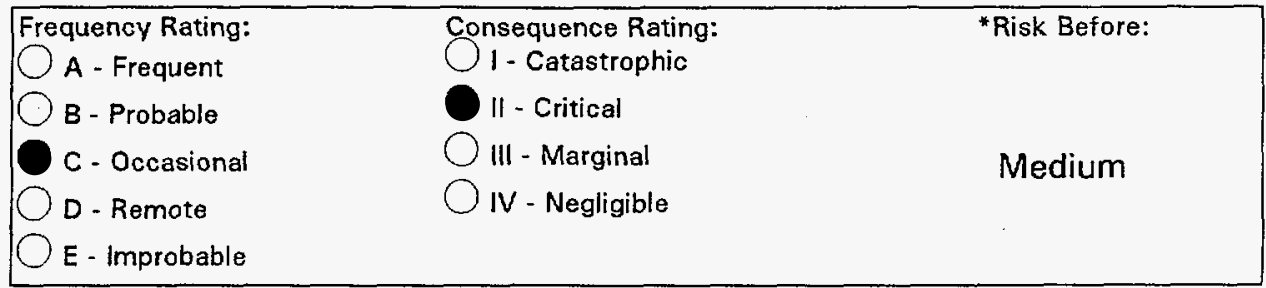

Frequency Rating:

A - Frequent

B - Probable

Oc - Occasional

O - Remote

E - Improbable

\section{Consequence Rating:}

O1 - Catastrophic

II - Critical

III - Marginal

OIV - Negligible

Extremely Low Risk After:

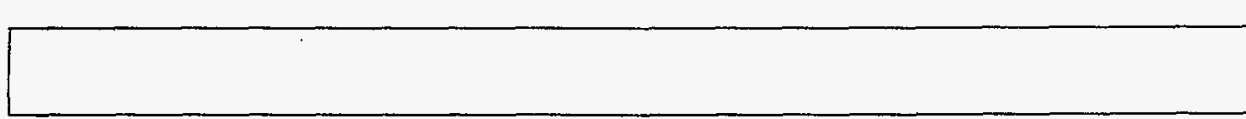

Underground Mapping of the ECRB, 19jun98, page4. 


\section{DI: BAB000000-01717-0200-0004; Rev 02}

\section{Mitigation Feature}

Scenario Number:

U15103 Mitigation Number: 1

Scenario:

Personnel receive electrical shock(s) from defective electrical equipment that shorts/burns out or from severed electrical wire/connectors.

Mitigation Feature: All electrical equipment used on the mapping floor shall be connected through a $\mathrm{GFCl}$ receptacle.

Documentation: Underground Mapping of the ECRB, 19jun98, page4.

Remarks: $\quad$ JSA developed. Part of mapping floor training.

Mitigation type: $\quad$\begin{tabular}{|l|} 
O Design Feature \\
Safety Device \\
$\bigcirc$ Warning Device \\
$\bigcirc$ Procedure \\
Training
\end{tabular}

\section{Mitigation Tracking}

Mitigation Implementation

Status:

Comments:
Not Implemented

Implemented

Appendix A

Page UI5103.1 


\section{DI: BAB000000-01717-0200-0004, Rev 02}

\section{Mitigation Feature}

Scenario Number: $\quad$ U15103 Mitigation Number: 2

Scenario: $\quad$ Personnel receive electrical shock(s) from defective electrical equipment that shorts/burns out or from severed electrical wire/connectors.

Mitigation Feature:

Electrical power boxes, equipment and power lines will only be activated when the mapping floor is seated on the tunnel rails (creates an extensive metal to metal grounding to the tunnel rail system).

Documentation: Underground Mapping of the ECRB, 19jun98, page4.

Remarks: $\quad$ JSA developed. Part of mapping floor training.

Mitigation type:
Design Feature
Safety Device
Warning Device
Procedure
Training

\section{Mitigation Tracking}

\section{Mitigation Implementation Status:}

Comments:

\section{Appendix A}

Page UI5103.2 


\section{DI: BAB000000-01717-0200-0004, Rev 02}

\section{Mitigation Feature}

Scenario Number:

Scenario:

Mitigation Feature:
Mitigation Number:

3

Personnel receive electrical shock(s) from defective electrical equipment that shorts/burns out or from severed electrical wire/connectors.

The mapping floor shall be grounded to the tunnel ground system (e.g., positively bonded by a ground cable and clamp to the tunnel grid wire.

Documentation:

Remarks:

Mitigation type:

Design Feature

Safety Device

Warning Device

Procedure

Training

\section{Mitigation Tracking}

Mitigation

Implementation

Status:

Comments:
Not Implemented

Implemented

Appendix $A$

Page UI5103.3 


\section{Scenario Description and Analysis}

\section{Approved}

Scenario Number:

Revision Number:

Revision Date:

\begin{tabular}{|l|}
\hline U15:05 \\
\hline 02 \\
\hline $12: 7 / 98$ \\
\hline
\end{tabular}

SSA

East-West Drift

Scenario:

Instriled electrical outlets/access panels in the tunnel and/or electrical connectors/cables are dar. ged when the mapping floor is moved.

Cause, Failure, or Hazardous Event:

Electrical cables are left connected to tunnel utility outlets or panels when the mapping floor is rioved.

Risk Assignment

Before Considering Mitigations:

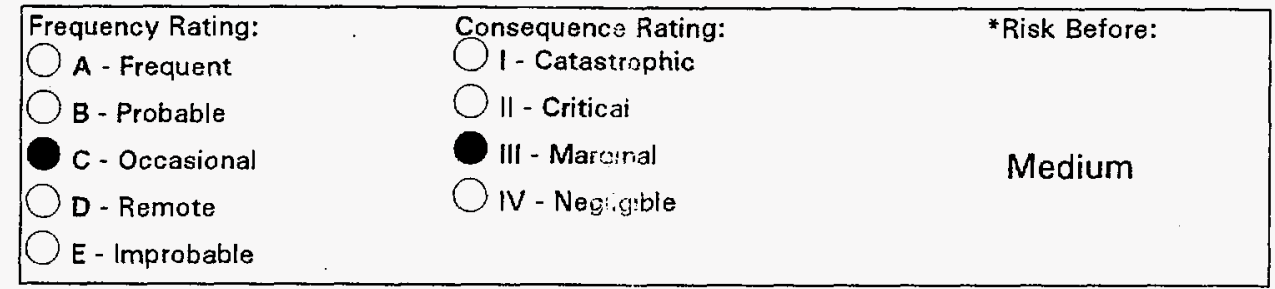

Risk Designation

After

Applying

Mitigations:

Frequency Rating:
A - Frequent
B - Probable
C - Occasional
D - Remote
E - Improbable

Consequence Rating:

1- Catastrophic

OII - Critical

III - Marginal

OIV - Negligible

Risk After:

Extremely Low

Related Scenarios:

Prevention

Mitigation

Documentation: 
DI: BAB000000-01717-0200-0004, Rev 02

\section{Mitigation Feature}

Scenario Number: $\quad$ U15105 Mitigation Number: 1

Scenario: $\quad$ Installed electrical outlets/access panels in the tunnel and/or electrical connectors/cables are damaged when the mapping floor is moved.

Mitigation Feature: Electrical power cables to the mapping floor will be disconnected and the cables stored prior to raising the mapping floor.

\section{Documentation:}

Remarks:

Mitigation type:
Design Feature
Safety Device
Warning Device
Procedure
Training

\section{Mitigation Tracking}

Mitigation

Implementation

Status:

Comments:
Not Implemented

$\bigcirc$ implemented 


\section{DI: BAB000000-01717-0200-0004, Rev 02}

\section{Mitigation Feature}

Scenario Number:

U15105 Mitigation Number:

2

Scenario:

Installed electrical outlets/access panels in the tunnel and/or electrical connectors/cables are damaged when the mapping floor is moved.

Mitigation Feature:

Upon cessation of scientific/survey work on the mapping floor in preparation for floor movement, the mapping floor supervisor will verify that the electrical cables are disconnected and stored.

Documentation:

Remarks:

Mitigation type:
Design Feature
Safety Device
Warning Device
Procedure
Training

\section{Mitigation Tracking}

Mitigation Implementation Status:

\section{Comments:}

\section{Not Implemented}

implemented

\section{Appendix A}

Page UI5105.2 


\section{DI: BAB000000-01717-0200-0004, Rev 02}

\section{Mitigation Feature}

Scenario Number: U15105 Mitigation Number: 3

Scenario: $\quad$ Installed electrical outlets/access panels in the tunnel and/or electrical connectors/cables are damaged when the mapping floor is moved.

Mitigation Feature: Prior to movement, the train operator and/or brakeman will physically inspect the mapping floor to verify that no utility (e.g., electrical, compressed air hoses) connections have been left attached to tunnel resources and that no equipment protrudes into utility or conveyor spaces that could damage those systems, the mapping floor or personnel.

Documentation:

Remarks:

Mitigation type:
Design Feature
Safety Device
Warning Device
Procedure
Training

\section{Mitigation Tracking}

\section{Mitigation \\ Implementation \\ Status:}

\section{Not Implemented}

$\bigcirc$ Implemented

Comments:

\section{Appendix A \\ Page UI5105.3}

